\title{
Main aggregates and summary accounts
}

UK GDP and national income

(Tables 1.1, 1.2, 1.3, 1.4)

\section{Gross domestic product at current prices}

\section{The three approaches and the need for balancing}

Gross Domestic Product (GDP) is arguably the most important aggregate or summary indicator for purposes of economic analysis and comparisons over time. It measures total domestic activity and can be defined in three different ways:

- GDP is the sum of gross value added of the institutional sectors or the industries plus taxes and less subsidies on products (which are not allocated to sectors and industries). It is also the balancing item in the total economy production account

- GDP is the sum of final uses of goods and services by resident institutional units (actual final consumption and gross capital formation), plus exports and less imports of goods and services

- GDP is the sum of uses in the total economy generation of income account (compensation of employees, taxes on production and imports less subsidies, gross operating surplus and gross mixed income of the total economy)

This is also the basis of estimating GDP. The use of three different methods which, as far as possible, use independent sources of information avoids sole reliance on one source and allows greater confidence in the overall estimation process.

The resulting estimates however, like all statistical estimates, contain errors and omissions; we obtain the best estimate of GDP (that is, the published figure) by reconciling the estimates obtained from all three approaches. On an annual basis this reconciliation is carried out through the construction of the input-output supply and use tables for the years for which data are available, and for subsequent periods by carrying forward the level of GDP set by the annual balancing process by using the quarterly movements in production, income and expenditure indicators.

For years in which no input-output balance has been struck a statistical discrepancy exists between estimates of the total expenditure components of GDP and the total income components of GDP after the balancing process has been carried out. This statistical discrepancy is made up of two components which are shown in the accounts, namely:
- the statistical discrepancy (expenditure adjustment), which is the difference between the sum of the expenditure components and the definitive estimate of GDP, plus

- the statistical discrepancy (income adjustment), which is the difference between the sum of the income components and the definitive estimate of GDP (with sign reversed)

As outlined in the framework above, the different approaches to the measurement of GDP provide various breakdowns useful for a wide range of economic analyses. These approaches are described in more detail below.

\section{The income approach}

The income approach provides estimates of GDP and its 'income' component parts at current market prices. The sources and methods of this approach are described in detail in chapter 14 of National Accounts Concepts, Sources and Methods.'

As it suggests, the income approach adds up all income earned by resident individuals or corporations in the production of goods and services and is therefore the sum of uses in the generation of income account for the total economy (or alternatively the sum of primary incomes distributed by resident producer units).

However some types of income are not included - these are transfer payments like unemployment benefit, child benefit or state pensions. Although they do provide individuals with money to spend, the payments are made out of, for example, taxes and national insurance contributions. Transfer payments are a redistribution of existing incomes and do not themselves represent any addition to current economic activity. To avoid double counting, these transfer payments and other current transfers (for example, taxes on income and wealth) are excluded from the calculation of GDP although they are recorded in the secondary distribution of income account.

In the UK the income approach to GDP is obtained by summing together:

- gross operating surplus

- gross mixed income

- compensation of employees (wages and salaries and employers' social contributions)

- taxes on production and imports less any subsidies on production 
Mixed income is effectively the operating surplus of unincorporated enterprises owned by households, which implicitly includes remuneration for work done by the owner or other members of the household. This remuneration cannot be identified separately from the return to the owner as entrepreneur.

As most of these incomes are subject to tax, the figures are usually obtained from data collected for tax purposes by HM Revenue \& Customs. However, because there is some delay in providing good quality estimates by this method, other sources are used to provide initial estimates.

The operating surplus and mixed income are measures of profit that exclude any holding gains. Holding gains result when, although no new goods or services have been produced, the value of inventories and fixed assets has increased simply as the result of an increase in the price of the item.

The Office for National Statistics (ONS) aims to cover the UK economy as comprehensively as possible. It is recognised that some income is not declared to the tax authorities, and to allow for this adjustments are routinely made to the GDP income measure. In 2008 the adjustment for undeclared income was about, $\mathrm{f} 27.3$ billion, approximately 1.9 per cent of GDP.

Although the income approach cannot be used to calculate chained volume measures directly (because it is not possible to separate income components into prices and quantities in the same way as for goods and services) some estimates are obtained indirectly. The expenditure-based GDP deflator at market prices (also known as the index of total home costs) is used to deflate the current market price estimates to provide a chained volume measure of the total income component of GDP for balancing purposes.

Data on the income components can be found in Table 1.2.

\section{The expenditure approach}

The expenditure approach measures total expenditure on finished or final goods and services produced in the domestic economy or, alternatively, the sum of final uses of goods and services by resident institutional units less the value of imports of goods and services.

The total is obtained from the sum of final consumption expenditure by households, non-profit institutions serving households and government on goods and services, gross capital formation (capital expenditure on tangible and intangible fixed assets, changes in inventories and acquisitions less disposals of valuables) and net exports of goods and services.
This approach can be represented by the following equation:

$$
\mathrm{GDP}=\mathrm{C}+\mathrm{G}+\mathrm{I}+\mathrm{X}-\mathrm{M}
$$

Where: $\quad \mathrm{C}=$ final consumption expenditure by households and NPISH sectors,

$\mathrm{G}=$ government consumption expenditure,

I = investment or gross capital formation,

$\mathrm{X}=$ exports and $\mathrm{M}=$ imports.

The data for these categories are estimated from a wide variety of sources including expenditure surveys, the government's internal accounting system, surveys of traders and the administrative documents used in the importing and exporting of some goods.

To avoid double counting, in this approach it is important to classify consumption expenditures as either final or intermediate. Final consumption involves the consumption of goods purchased by or for the ultimate consumer or user.

These expenditures are final because the goods are no longer part of the economic flow or being traded in the market place. Intermediate consumption on the other hand is consumption of goods and services which are used or consumed in the production process. Gross capital formation is treated separately from intermediate expenditure as the goods involved are not used up within the production process in an accounting period.

Exports include all sales to non-residents, and exports of both goods and services have to be regarded as final consumption expenditure, since they are final as far as the UK economy is concerned.

Imports of goods and services are deducted because although they are included directly or indirectly in final consumption expenditure they are not part of domestic production. What remains is what has been produced in the UK - gross domestic product using the expenditure approach.

Data on the current price expenditure components can be found in Table 1.2.

As well as GDP at current prices, the expenditure approach is used to estimate chained volume measures of GDP. The chained volume measure shows the change in GDP after the effects of inflation have been removed (see 'UK GDP Chained Volume Measures'). 
GDP at market prices ( $\mathrm{f}$ million)

\begin{tabular}{lrr} 
& Current prices & Chained volume measures \\
\hline 1996 & 781,726 & $1,019,337$ \\
1997 & 830,013 & $1,054,232$ \\
1998 & 879,152 & $1,094,704$ \\
1999 & 928,871 & $1,134,723$ \\
2000 & 976,282 & $1,185,305$ \\
2001 & $1,021,625$ & $1,222,650$ \\
2002 & $1,075,368$ & $1,255,142$ \\
2003 & $1,139,441$ & $1,299,381$ \\
2004 & $1,202,370$ & $1,337,782$ \\
2005 & $1,254,292$ & $1,365,685$ \\
2006 & $1,328,597$ & $1,401,290$ \\
2007 & $1,405,796$ & $1,449,861$ \\
2008 & $1,433,870$ & $1,433,871$ \\
2009 & $1,393,854$ & $1,371,163$ \\
2010 & $1,458,452$ & $1,395,312$ \\
\hline
\end{tabular}

The reference year for the chained volume measure series in this edition of the Blue Book is 2008; the chained volume measure of GDP for 2008 is referenced to the annual current price estimate of GDP for 2008.

Two methods are used to remove the effects of inflation to obtain these chained volume measures. For some series, price indices for particular goods and services - such as components of the consumer prices index (CPI) or the producer price index (PPI) - are used to 'deflate' the current price series. For other series, chained volume measures are assumed to be proportional to the volume of goods or services. Chained volume measures of GDP and its main expenditure components can be found in Table 1.3; the calculation of these chained volume measures are explained below.

\section{The production approach}

The production approach to the estimation of GDP, which is also referred to as the output approach, looks at the contribution to production of each economic unit; that is the value (at basic prices) of their total output less the value of the inputs used up in the production process. The sum of these gross values added, plus taxes and less subsidies on products for all producers, is GDP at market prices: the production account balancing item. The following paragraphs give a brief overview of the methodology. It should be noted that the production approach concentrates on the basic price concept.

In theory, chained volume measures of value added should be estimated by double deflation; that is, deflating separately the inputs and the outputs of each economic unit (valued in chained volume measures) and then subtracting one from the other. But, because it is hard to get reliable data on intermediate consumption, double deflation is only used in the estimation of output for the agriculture and electricity industries. So for most industries, movements in the chained volume measures for gross value added are estimated by the use of output series. For industries whose outputs are goods, output can be estimated from the physical quantities of goods produced or from the value of output deflated by an index of price.

Apart from the use of output to estimate chained volume measures of value added, which accounts for around 80 per cent of the total of the production measure, a number of other kinds of indicator might be used as a proxy for the change in gross value added. For example, they may be estimated by changes in inputs, where the inputs chosen may be materials used, employment or some combination of these.

In the short-term it is reasonable to assume that movements in value added can be measured this way. However, changes in the ratio of output and inputs to gross value added can be caused by many factors: new production processes, new products made and inputs used, and changes in inputs from other industries will all occur over time. Aggregated over all industries the impact of these changes will be lessened. In the longer term all indicators are under constant review, with more suitable ones being used as they become available.

The estimate of gross value added for all industries (the proxy for the quarterly production approach to GDP) is finally obtained by combining or 'weighting together' the estimates for each industrial sector according to its relative importance (as established in the Input-Output Supply and Use tables). For each year these weights are based on supply and use data for the immediately preceding year, except for the most recent years where the weights are based on supply and use data for 2008 (see 'UK GDP Chained Volume Measures'). Data can be found in Table 2.4 .

\section{Headline GDP}

The chained volume measure of gross domestic product at market prices provides the key indicator of change in the economy: this is sometimes called 'headline' GDP. The chained volume measure of gross value added at basic prices (GVA), another useful short-term indicator of growth in the economy, is the headline measure for the production approach. It is compiled in a way which is relatively free of short-term fluctuations due to uncertainties of timing. The construction of chained volume measures of gross domestic product at factor cost however, requires an adjustment for the relevant taxes and subsidies which can be subject to erratic changes. As a result the factor cost measure is less suitable as an indicator of short-term movements in the economy. 
The figure below shows the distinction between market prices, basic prices and factor cost measures.

\begin{tabular}{|l|l|}
\hline ESA95 code & \\
\hline & Gross domestic product, at market prices \\
\hline D.211 & Less value added taxes (VAT) on products \\
\hline D.212, D.214 & $\begin{array}{l}\text { Less other taxes on products } \\
\text { (for example, alcohol duty) }\end{array}$ \\
\hline D.31 & Plus subsidies on products \\
\hline D.29 & $\begin{array}{l}\text { Gross value added, at basic prices } \\
\text { less taxes on production other than taxes } \\
\text { vehicle excise duty paid by businesses and } \\
\text { operating licences) }\end{array}$ \\
\hline & \begin{tabular}{l} 
Gross value added, at factor cost \\
\hline
\end{tabular}
\end{tabular}

GDP at market prices includes taxes on production, whilst GDP at basic prices includes only those taxes on production, such as business rates which are not taxes on products and GDP at factor cost excludes all taxes on production. A more detailed explanation of taxes follows.

Taxes

Taxes on production and imports including taxes on products (D.2), along with subsidies (D.3) (which can be regarded as negative taxes) make up the factor cost adjustment which represents the difference between GDP at market prices (sum of final expenditures) and GVA at factor cost (sum of incomes). This adjustment has to be added to the sum of incomes to obtain GDP at market prices. The basic price adjustment, which is the sum of taxes on products (D.21) less subsidies on products (D.31), is the difference between GVA at basic prices and GDP at market prices. Details of the taxes which comprise taxes on production are included in Table 11.1.

Taxes on production and imports (D.2) are taxes paid during the production or import of goods and services. They are paid irrespective of whether profits are made. They comprise taxes on products (D.21) and other taxes on production (D.29).

Taxes on products (D.21) are taxes paid per unit of good or service produced, sold, leased, transferred, exported or imported. They are included in the prices paid to suppliers of goods and services, so they are included in intermediate consumption at purchasers' prices (except for deductible VAT). Fuel duty is an example.

Deductible VAT differs from other taxes on products. It is levied like other taxes on products but producers are reimbursed by government for the amount they pay when goods and services are bought. Intermediate consumption at purchasers' prices is the price paid less deductible VAT refunded. The value of sales or production at producers' prices also excludes any deductible VAT charged.

Suppliers are required to pay to government any taxes on products included in their prices. So the supplier's net revenue from selling the good is the selling price less the taxes on products included in the selling price. This is the basic price. It is the price at which market output is measured since it represents the producers' actual revenue.

Other taxes on production (D.29) are taxes which producers have to pay but they are not paid when goods and services are bought and so are not included in intermediate consumption. They are levied separately and are usually linked to the use of fixed capital or to the right to undertake certain regulated activities. Examples are non-domestic rates, vehicle excise duty, and various licence fees where the fee is much higher than the cost of administering the licence and so, in effect, is classified as taxation.

\section{Other aggregates - gross national income and gross national disposable income}

In the discussions so far we have yet to consider the measure which represents the total disposable income of the country's residents. Gross national income (GNI) represents the total income of UK residents and is the balancing item of the UK allocation of primary income account. It can also be derived from GDP by adding net employment income and net property income from the rest of the world. However there are two other areas which affect UK residents' command over resources

First, there are flows into and out of the country which are not concerned with economic production. These are current transfers from abroad and current transfers paid abroad. They include transactions with the European Union, overseas aid and private gifts. An estimate of gross national disposable income (GNDI) is reached by adjusting GNI by the amount of net income received. GNI and GNDI are shown in Table 1.1.

Second, disposable income is affected by the terms of trade effect. Some of the expenditure by UK residents is on imported goods and services; some of the income earned by residents is from exports of goods and services. If UK export prices fall relative to the price of imports then the terms of trade effect would move against the UK; that is, residents would have to sell more exports to be able to continue to buy the same amount of imports. The purchasing power of UK residents would be diminished to this extent. Similarly, if UK export prices rose relative to prices of imports then the effect would be opposite: the purchasing power of residents would rise. An adjustment is made specifically for the terms of trade effect in 
calculating the chained volume measure of GNDI, also shown in Table 1.1.

\section{UK GDP chained volume measures (Tables 1.1, 1.3, 1.4)}

When looking at the change in the economy over time, the main concern is usually whether more goods and services are actually being produced now than at some time in the past.

Over time, changes in current price GDP show changes in the monetary value of the components of GDP. As these changes in value can reflect changes in both price and volume, it is difficult to establish how much of an increase in the series is due either to increased activity in the economy or to an increase in the price level. It is therefore useful to measure GDP in real terms (that is, excluding price effects) as well as at current prices. In most cases the revaluation of current price data to remove price effects (known as deflation) is carried out by using price indices such as component series of the consumer prices index or producer price index to deflate current price series at a detailed level of disaggregation. In the 2003 edition of the Blue Book a new method of measuring GDP in real terms, annual chain-linking, was introduced to replace fixed base chain-linking which was used in previous editions of the Blue Book. The real GDP time series produced by annual chain-linking are referred to as chained volume measures.

In the UK economic accounts the expenditure approach is used to provide current price and chained volume measures of GDP. Because of the difficulties in accounting for changes in labour productivity it is not possible to obtain direct chained volume measures of GDP from the income data. However, an approximate aggregate measure is calculated by deflating the current price estimates using the GDP deflator derived from the expenditure measure for balancing purposes. The production measure of GDP is largely based on output measures.

\section{The introduction of annual chain-linking}

The fixed-base chain-linking method, which was used in editions of the Blue Book prior to 2003, produced 'constant price' estimates of GDP whereby the price structure prevailing in 1995 was used to compile data from 1994 onwards. For years prior to 1994 more appropriate pricing structures were used and, in order to link all of the 'constant price' estimates to produce continuous time series, a process of chain-linking was used whereby blocks of constant price data with different price bases were linked together. In the link years, figures were calculated with reference to two consecutive base years to obtain a linking factor so that the whole time series could be shown with reference to the latest base year. This system of fixed-base chain-linking is described in more detail at pages 36 to 38 of the 2002 edition of the Blue Book.
In the 2003 edition of the Blue Book, the fixed-base chainlinking method was replaced with an annual chain-linking process which produces 'chained volume measures' of GDP. Chained volume measures are calculated by applying the price structure prevailing in the previous year for each year, except the most recent available years where chained volume measures are calculated by applying the price structure prevailing in 2008 . The year 2008 is therefore the 'latest base year' for chained volume measures published in this edition of the Blue Book. Thus estimates for 2009 and 2010 are based on 2008 prices, estimates for 2008 are based on 2007 prices and so on. These 'previous years prices' data are chain-linked to produce continuous time series called 'chained volume measures', in a similar fashion to the fixed-based chain-linking described in the above paragraph.

These chained volume measure series are shown in $\mathrm{f}$ million and referenced onto the 'latest base year' which is 2008 in this edition of the Blue Book. Current price data therefore equals chained volume measures annually in 2008. The process of annually chain-linking 'previous years prices' data onto a continuous time series referenced onto the latest base year results in a loss of additivity in the annual data prior to the latest base year. Thus chained volume measures prior to 2008 are non-additive in this edition of the Blue Book. Usually the 'latest base year' and therefore the 'reference year' will move forward by one year.

In the expenditure measure of GDP all of the components are annually chain-linked, as described above, and the chained volume measure of total GDP is aggregated from these. The output approach involves weighting together the detailed components using the contribution to current price GVA (or weight) in the immediately preceding year and annually chain-linking to produce a continuous time series. The application of annual chain-linking to the output measure of GDP is described in detail in an article published in the October 2001 edition of Economic Trends. ${ }^{2}$

Annual chain-linking provides more accurate measures of growth in the economy than that provided by the old method of fixed-base chain-linking because more up to date, and therefore more appropriate, price structures are used. The move to annual chain-linking is also consistent with international guidelines laid down in the System of National Accounts 1993 (SNA93).

\section{Index numbers and price indices}

Some chained volume measure series are expressed as index numbers in which the series are simply scaled proportionately to a value of 100 in the reference year. These index numbers are volume indices of the 'base weighted' or 'Laspeyres' form 
(see chapter 2 of National Accounts Concepts, Sources and Methods ${ }^{1}$ ). Aggregate price indices are of the 'Paasche' or 'current-weighted' form. They are generally calculated indirectly by dividing the current price value by the corresponding chained volume measure and multiplying by 100 . Examples are the GDP deflator and the households' consumption deflator.

Value indices are calculated by scaling current price values proportionately to a value of 100 in the reference year. By definition such a value index, if divided by the corresponding volume index and multiplied by 100 , will give the corresponding price index.

\section{Population, employment and GDP per head (Table 1.5)}

Population and employment data are supplementary to the system of accounts. The estimated population of the UK is as at 30 June and includes all those resident in the UK, whatever their nationality. They include members of both UK and non-UK armed forces and their dependants stationed in the UK and exclude members of H.M. armed forces stationed in the rest of the world. This is recognised as not being in strict accord with ESA95 requirements, which are for all UK armed forces and dependants, wherever stationed, to be included and all non-UK ones to be excluded. At present, this is the most appropriate estimate available; it is used to calculate GDP per head.

The total employment data are from the UK Labour Force Survey (LFS) which is recognised as the most appropriate source for coherent national aggregate labour market estimates. The LFS is a household survey which uses definitions which are consistent with the International Labour Organisation recommendations and have been adopted by all EU member countries. The coverage of the LFS is people living in private households and, from 1992, student halls of residence and NHS accommodation; it is not precisely consistent with either the home population data or the ESA95 requirements.

The employment data in the table are estimates of people according to their economic and employment status. They are not comparable with estimates of jobs, as shown in Table 2.5, as some people have more than one job. The total employment figures include people on government sponsored training and employment programmes and unpaid family workers.

\section{UK summary accounts (Tables 1.6 .0 - 1.6.9)}

The UK summary accounts show the full set of accounts for the UK total economy. The accounts comprise of the goods and services account, the production account, the distribution and use of income account and the accumulation accounts. The structure of the accounts is explained in the introduction.

\section{UK summary accounts by sector}

\section{(Tables 1.7.1 - 1.7.9)}

\section{The framework}

As can be seen in Table 1.7, the UK sector accounts can be used to show the economic accounting framework in considerable detail by elaborating the accounts in three different dimensions:

- the institutional sectors

- the types of transaction

- the national and sector balance sheets

The institutional sectors

The first dimension involves the breakdown of the current account into institutional sectors grouped broadly according to their roles in the economy. Examples of these roles are: income distribution, income redistribution, private consumption, collective consumption, investment, financial intermediation, etc. Most units have more than one role but a natural classification is to distinguish between corporations, government and households. The rest of the world sector is also identified as having a role although it is obviously not part of the domestic economy.

\section{The types of transaction}

The second dimension is that of the type of transaction which relates to the particular account within which the transaction appears. These can be grouped broadly according to purpose, whether current, capital or financial.

\section{Summary of the UK institutional sectors}

\begin{tabular}{|c|c|c|}
\hline Sectors and sub-sectors & ESA95 & code \\
\hline Non-financial corporations & S.11 & \\
\hline Public & & S.11001 \\
\hline National private and foreign controlled & & S.11002/3 \\
\hline Financial corporations & S.12 & \\
\hline Central bank & & S.121 \\
\hline Other monetary financial institutions & & S.122 \\
\hline Other financial intermediaries & & S.123 \\
\hline Financial auxiliaries & & S.124 \\
\hline $\begin{array}{l}\text { Insurance corporations and } \\
\text { pension funds }\end{array}$ & & S.125 \\
\hline General government: & S.13 & \\
\hline Central government & & S.1311 \\
\hline Local government & & S.1313 \\
\hline Households & S.14 & \\
\hline $\begin{array}{l}\text { Non-profit institutions serving } \\
\text { households (NPISH) }\end{array}$ & S.15 & \\
\hline Rest of the world & S.2 & \\
\hline
\end{tabular}




\section{The balance sheets}

To complete the full set of accounts the system includes balance sheets and a reconciliation of the changes that have brought about the change between the beginning and the end of the period. At present the UK does not compile the latter except for the general government sector which are available in the ONS public sector finances release.

In theory the net lending or borrowing from the capital account for each sector should equal the net borrowing or lending from the financial account. In practice, because of errors and omissions in the accounts, a balance is rarely achieved and the difference is known as the statistical discrepancy although, across all accounts, when an InputOutput balance is available, these sum to zero. Consolidating the current and accumulation accounts would provide a balanced account which would look like many of the presentations of commercial accounts.

\section{Spurious accuracy and rounding to the nearest f million}

One final point must also be made about the reliability of the statistics. In most of the published tables no attempt is made to round estimates beyond the nearest $f$ million. In some instances this shows figures which appear to have more precision than evidence warrants.

The reasons for this presentation are as follows:

- rounded figures can distort differences over time or between items

- some of the estimates in the tables are fairly precise and, if such an estimate is small, rounding would unnecessarily distort what it shows; yet if such series were not rounded to the nearest $f$ million the major aggregates of which they are components would appear precise even though other components were heavily rounded

- not rounding beyond the nearest $f$ million aids users who prepare derived statistics, by avoiding the accumulation of rounding errors which can occur when a number of rounded numbers are manipulated

- in presenting numbers to the nearest $f$ million, the rounding is usually such that the components add to the total at current prices, so that the accounts balance. In particular the quarterly estimates, both before and after seasonal adjustment, add up to the calendar year totals. However, there are some small differences between the sum of component series and the total shown, due to rounding

\section{Changes since last year's Blue Book}

An analysis of revisions in current prices since last year's Blue Book is shown in Table B.

The revisions to the aggregate GDP levels and growth from 1997 to 2009 are as a result of balancing of those years through the supply and use framework and the incorporation of annual benchmark survey estimates, especially the Annual Business Survey. In this year's Blue Book, 1997 to 2008 have been re-balanced and 2009 has been balanced for the first time. More details can be found in chapter 2 of this publication. Current price data have been revised from 1997.

Chained volume estimates and index numbers have been updated as the reference year has been advanced from 2006 to 2008. This change has an impact on the entire time series for affected series.

\section{References}

1 Office for National Statistics (1998) National Accounts Concepts, Sources and Methods. The Stationery Office; London.

http://www.ons.gov.uk/ons/rel/naa1-rd/national-accountsconcepts--sources-and-methods/1998-release/nationalaccounts-concepts--sources-and-methods.pdf

2 Tuke A and Reed G (2001) The Effects of Annual Chainlinking on the Output Measure of GDP. Economic Trends No. 575.

http://www.ons.gov.uk/ons/rel/elmr/economic-trends-discontinued-/no--575--october-2001/the-effects-of-annualchain-linking-on-the-output-measure-of-gdp.pdf

\section{Blue Book 2011 Articles}

Clancy G (2011) Improvements to Household Expenditure Estimates - Q2 2011 http://www.ons.gov.uk/ons/rel/consumer-trends/blue-book2011--improvements-to-household-expenditure-estimates/ q2-2011/art---blue-book-2011--improvements-to-householdexpenditure-estimates.html

Drew S and Dunn M (2011) Blue Book 2011: Reclassification of the UK Supply and Use tables http://www.ons.gov.uk/ons/rel/input-output/input-outputsupply-and-use-tables/reclassification-of-the-uk-supply-anduse-tables/index.html

Drew S (2011) Deflation Improvements in the UK National Accounts http://www.ons.gov.uk/ons/rel/naa1-rd/national-accountsconcepts--sources-and-methods/august-2011/deflationimprovements-in-the-uk-national-accounts.pdf 
Duff H (2011) Blue Book 2011: Improvements to GDP(O), loS and IOP

http://www.ons.gov.uk/ons/dcp171766_229044.pdf

Duff S (2011) Improving the coverage of derivatives in the National Accounts and Balance of Payments

http://www.ons.gov.uk/ons/rel/naa1-rd/united-kingdomeconomic-accounts/improving-the-coverage-of-derivatives-datain-the-national-accounts-and-balance-of-payments/artimproving-the-coverage-of-derivatives-data-in-na-and-bop.html

Economic Review: September 2011

http://www.ons.gov.uk/ons/dcp171766_237189.pdf

Everett G (2011) Content of Blue Book 2011

http://www.ons.gov.uk/ons/rel/naa1-rd/united-kingdomnational-accounts/2011-edition/content-of-blue-book.pdf

Everett G (2011) Historic National Accounts Data Proposals for Blue Book 2011

http://www.ons.gov.uk/ons/rel/naa1-rd/united-kingdomnational-accounts/historic-national-accounts-data-proposalsfor-blue-book-2011/ard-historic-national-accounts-dataproposals-fopr-blue-book-2011.pdf

Everett G (2011) Methods Changes in Blue Book 2011 http://www.ons.gov.uk/ons/rel/naa1-rd/united-kingdomnational-accounts/method-changes-in-blue-book-2011/ ard-method-changes-in-blue-book-2011.pdf
Lee P, Myers M (2011) Impact of changes in National Accounts and economic commentary for Q2 2011

http://www.ons.gov.uk/ons/rel/naa2/quarterly-nationalaccounts/impact-of-changes-in-national-accounts-andeconomic-commentary-for-q2-2011/ard-impact-of-changes-inthe-national-accounts-and-economic-commentary-for-2011quarter-2.pdf

McLaren C (2011) Blue Book 2011: Improvements to gross capital formation estimates http://www.ons.gov.uk/ons/dcp171766_229111.pdf

National Accounts Publication Timetable: September http://www.ons.gov.uk/ons/media-centre/statements/nationalaccounts-publication-timetable.html

\section{Other articles}

Simkins A (2010) Financial Crisis and Recession: How ONS has addressed the Statistical and Analytical Challenge. http://www.ons.gov.uk/ons/rel/elmr/economic-and-labourmarket-review/no--1--january-2010/financial-crisis-andrecession--how-ons-has-addressed-the-statistical-andanalytical-challenges.pdf 


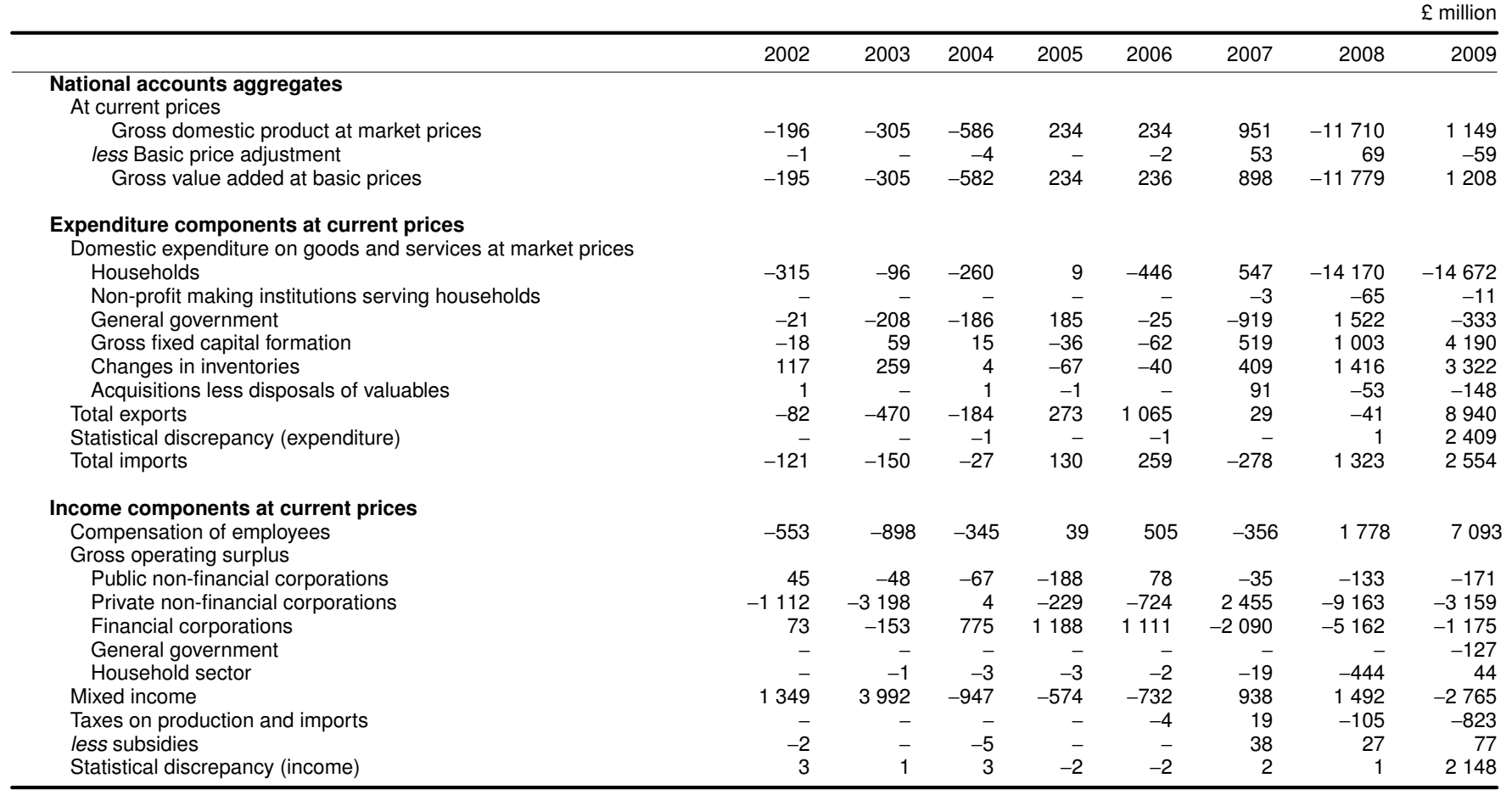




\begin{tabular}{|c|c|c|c|c|c|c|c|c|c|}
\hline & & & 1997 & 1998 & 1999 & 2000 & 2001 & 2002 & 2003 \\
\hline & \multicolumn{9}{|l|}{ INDICES $(2008=100)$} \\
\hline & \multicolumn{9}{|l|}{ VALUES AT CURRENT PRICES } \\
\hline B. $1^{*} \mathrm{~g}$ & \multicolumn{9}{|l|}{$\begin{array}{l}\text { Gross domestic product at current market prices } \\
\text { ("money GDP") }\end{array}$} \\
\hline \multirow[t]{2}{*}{ B. $1 \mathrm{~g}$} & Gross value added at current basic prices & YBEX & 57.6 & 60.9 & 64.1 & 67.3 & 70.7 & 74.5 & 79.0 \\
\hline & \multicolumn{9}{|l|}{ CHAINED VOLUME MEASURES } \\
\hline B. $1^{*} \mathrm{~g}$ & Gross domestic product at market prices & YBEZ & 73.5 & 76.3 & 79.1 & 82.7 & 85.3 & 87.5 & 90.6 \\
\hline B. $6^{*} \mathrm{~g}$ & Gross national disposable income at market prices & YBFP & 71.9 & 75.6 & 77.5 & 80.8 & 84.3 & 87.5 & 90.6 \\
\hline \multirow[t]{3}{*}{ B. $1 \mathrm{~g}$} & Gross value added at basic prices & CGCE & 73.3 & 76.3 & 79.2 & 82.9 & 85.3 & 87.3 & 90.5 \\
\hline & \multicolumn{9}{|l|}{ PRICES } \\
\hline & Implied deflator of GDP at market prices & YBGB & 78.7 & 80.3 & 81.9 & 82.4 & 83.6 & 85.7 & 87.7 \\
\hline
\end{tabular}

\section{VALUES AT CURRENT PRICES (£ million)}

Gross measures (before deduction of fixed capital consumption) at current market prices

B.1*g Gross domestic product ("money GDP") Employment, property and entrepreneurial income from the rest of the world (receipts less payments) Subsidies (receipts) less taxes (payments) on products from/to the rest of the world

B.5 ${ }^{\star} \mathrm{g}$ Gross national income (GNI)

D.5,6,7 Current transfers from the rest of the world

\section{B. ${ }^{\star} \mathrm{g}$ Gross national disposable income}

\section{Adjustment to current basic prices}

B.1* G Gross domestic product (at current market prices)

-D.21 Adjustment to current basic prices

+D.31 (less taxes plus subsidies on products)

B.1g Gross value added (at current basic prices)

Net measures (after deduction of fixed

-K.1 capital consumption) at current market prices

B. $1^{*} \mathrm{n} \quad$ Net domestic product

B. $5^{\star} \mathrm{n}$ Net national income

B.6*n Net national disposable income

\begin{tabular}{|c|c|c|c|c|c|c|c|}
\hline YBHA & 830013 & 879152 & 928871 & 976282 & 1021625 & 1075368 & 1139441 \\
\hline YBGG & 325 & 11799 & -1042 & 1963 & 9425 & 18503 & 17770 \\
\hline $\begin{array}{l}\text { QZOZ } \\
\text { IBJL }\end{array}$ & $\begin{array}{r}-2919 \\
208\end{array}$ & $\begin{array}{r}-3651 \\
241\end{array}$ & $\begin{array}{r}-3438 \\
338\end{array}$ & $\begin{array}{r}-4098 \\
335\end{array}$ & $\begin{array}{r}-3920 \\
582\end{array}$ & $\begin{array}{r}-2890 \\
519\end{array}$ & $\begin{array}{r}-2596 \\
592\end{array}$ \\
\hline ABMX & 827623 & 887543 & 924729 & 974484 & 1027712 & 1091500 & 1155207 \\
\hline YBGF & -2816 & -4764 & -4224 & -6016 & -3182 & -6500 & -7835 \\
\hline $\mathrm{NQCO}$ & 824807 & 882779 & 920505 & 968468 & 1024530 & 1085000 & 114 \\
\hline
\end{tabular}

\section{CHAINED VOLUME MEASURES}

(Reference year 2008, £ million)

Gross measures (before deduction of fixed capital consumption) at market prices

B.1* Gross domestic product

TGL Terms of trade effect ("Trading gain or loss")

GDI Real gross domestic income

Real employment, property and entrepreneurial income

D.1+D.4 from the rest of the world (receipts less payments)

Subsidies (receipts) less taxes (payments) on

$-\mathrm{D} .21+\mathrm{D} .31$

+ D. $29-$ D. 39 products from/to the rest of the world

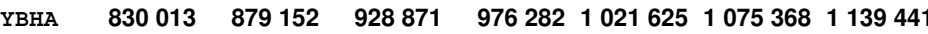

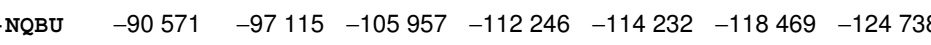

$\begin{array}{lllllllll}\text { ABML } & 739442 & 782037 & 822914 & 864036 & 907393 & 956899 & 1014703\end{array}$

-NQAE

$\cos$ $\begin{array}{lllllllll}\text { NSRX } & 732444 & 788583 & 819222 & 863233 & 911916 & 969586 & 1029604\end{array}$ $\begin{array}{lllllllll}\text { NQCP } & 729628 & 783819 & 814998 & 857217 & 908734 & 963086 & 1021769\end{array}$

B.5 ${ }^{\star} \mathrm{g}$ Gross national income (GNI) Real current transfers from the rest of the world
D.5,6,7 receipts less payments)

B.6*g Gross national disposable income

\begin{tabular}{|c|c|c|c|c|c|c|c|}
\hline $\begin{array}{l}\text { ABMI } \\
\text { YBGJ }\end{array}$ & $\begin{array}{r}1054232 \\
-3113\end{array}$ & $\begin{array}{r}1094704 \\
129\end{array}$ & $\begin{array}{r}1134723 \\
2257\end{array}$ & $\begin{array}{r}1185305 \\
-1803\end{array}$ & $\begin{array}{r}1222650 \\
-1764\end{array}$ & $\begin{array}{r}1255142 \\
5106\end{array}$ & 129 \\
\hline YBGL & 1051119 & 1094833 & 1136980 & 1183502 & 1220886 & 1260248 & 1307 \\
\hline BGI & 411 & 14665 & -1274 & 2376 & 11246 & 21691 & \\
\hline & $\begin{array}{r}-3690 \\
263\end{array}$ & $\begin{array}{r}-4541 \\
300\end{array}$ & $\begin{array}{r}-4202 \\
413\end{array}$ & $\begin{array}{r}-4961 \\
406\end{array}$ & $\begin{array}{r}-4678 \\
695\end{array}$ & $\begin{array}{r}-3388 \\
608\end{array}$ & \\
\hline
\end{tabular}

YBGM 1048092110527911319081181318122815812791841325782 $\begin{array}{llllllll}- \text { YBGP } & -3561 & -5926 & -5164 & -7283 & -3797 & -7621 & -8995\end{array}$ YBGO 1044533109935311267451174034122436412715621316786

\section{Adjustment to basic prices}

B.1*g Gross domestic product (at market prices)

-D.21 Adjustment to basic prices

+D.31 (less taxes plus subsidies on products)

\section{B.1g Gross value added (at basic prices)}

Net measures (after deduction of fixed

-K.1 capital consumption) at market prices ${ }^{1}$

B. $5^{\star} \mathrm{n} \quad$ Net national income at market prices

B.6*n Net national disposable income at market prices

\footnotetext{
1 Capital consumption CVM estimates are still referenced to 2006. Estimates

for these series with CVM reference year 2008 will be published in the

"Capital Stocks and Capital Consumption" statistical bulletin in 2012.
}

ABMI 1054232109470411347231185305122265012551421299381

$\begin{array}{lllllllll}\text {-NTAQ } & -113807 & -115557 & -117819 & -121738 & -127 & 762 & -133997 & -137875\end{array}$

ABMM $\quad 941159 \quad 97960210173001064074109518111211841161561$

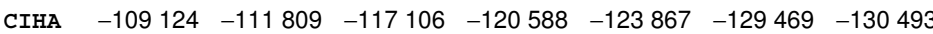

YBET $\quad 931298 \quad 98393810046861049287 \quad 109230311371851181327$ $\begin{array}{llllllllll}\text { BEY } & 927748 & 978 & 013 & 999526 & 1042001 & 1088519 & 1129564 & 1172330\end{array}$ 


\begin{tabular}{|c|c|c|c|c|c|c|c|c|c|}
\hline & & & 2004 & 2005 & 2006 & 2007 & 2008 & 2009 & 2010 \\
\hline & \multicolumn{9}{|l|}{ INDICES $(2008=100)$} \\
\hline & \multicolumn{9}{|l|}{ VALUES AT CURRENT PRICES } \\
\hline B. $1^{*} \mathrm{~g}$ & \multicolumn{9}{|l|}{ Gross domestic product at current market prices } \\
\hline \multirow[t]{2}{*}{ B. $1 \mathrm{~g}$} & Gross value added at current basic prices & YBEX & 83.4 & 87.0 & 92.2 & 97.6 & 100.0 & 97.9 & 101.3 \\
\hline & \multicolumn{9}{|l|}{ CHAINED VOLUME MEASURES } \\
\hline B. $1^{*} \mathrm{~g}$ & Gross domestic product at market prices & YBEZ & 93.3 & 95.2 & 97.7 & 101.1 & 100.0 & 95.6 & 97.3 \\
\hline B. $6^{*} \mathrm{~g}$ & Gross national disposable income at market prices & YBFP & 93.2 & 94.7 & 96.4 & 100.5 & 100.0 & 94.6 & 96.0 \\
\hline \multirow[t]{3}{*}{ B. $1 \mathrm{~g}$} & Gross value added at basic prices & $\mathrm{CGCE}$ & 93.0 & 95.1 & 97.5 & 101.0 & 100.0 & 95.4 & 97.1 \\
\hline & \multicolumn{9}{|l|}{ PRICES } \\
\hline & Implied deflator of GDP at market prices & YBGB & 89.9 & 91.8 & 94.8 & 97.0 & 100.0 & 101.7 & 104.5 \\
\hline
\end{tabular}

\section{VALUES AT CURRENT PRICES (£ million)}

Gross measures (before deduction of fixed capital consumption) at current market prices

B.1*g Gross domestic product ("money GDP") Employment, property and entrepreneurial income from the rest of the world (receipts less payr Subsidies (receipts) less taxes (payments) on products from/to the rest of the world

Other subsidies on production from/to the rest of the world

YBHA 1202370125429213285971405796143387013938541458452 $\begin{array}{llllllll}\text { YBGG } & 18029 & 21886 & 9512 & 21369 & 33136 & 20397 & 23039\end{array}$ $\begin{array}{llllllll}\text { QZOZ } & -1234 & -4260 & -4496 & -4731 & -4906 & -4238 & -5186\end{array}$ $\begin{array}{lllllll}592 & 3408 & 3221 & 2952 & 3051 & 3411 & 3032\end{array}$

B.5 ${ }^{\star} \mathrm{g}$ Gross national income (GNI)

D. Current transfers from the rest of the world (receipts less payments)

ABMX 1219759127532313368301425387146515214134171479337

B.6* Gross national disposable income

$\begin{array}{llllllll}- \text { YBGF } & -9645 & -11052 & -10610 & -11804 & -11912 & -14290 & -17937\end{array}$ NQCO 1210114126427113262201413583145324013991271461400

Adjustment to current basic prices

B.1* G Gross domestic product (at current market prices)

-D.21 Adjustment to current basic prices

+D.31 (less taxes plus subsidies on products)

B.1g Gross value added (at current basic prices)

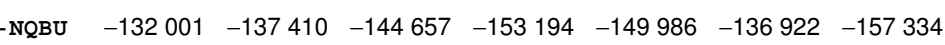
ABML $\quad 1070369111688211839401252602128388412569321301118$

Net measures (after deduction of fixed

-K.1 capital consumption) at current market prices

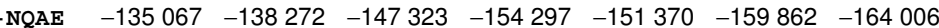

B. $1^{*} \mathrm{n} \quad$ Net domestic product

B. $5^{\star} \mathrm{n}$ Net national income

B. $6^{*} \mathrm{n} \quad$ Net national disposable income

\section{CHAINED VOLUME MEASURES}

(Reference year 2008, £ million)

Gross measures (before deduction of fixed capital consumption) at market prices

B.1*g Gross domestic product

TGL Terms of trade effect ("Trading gain or loss")

GDI Real gross domestic income

Real employment, property and entrepreneurial income

D.1+D.4 from the rest of the world (receipts less payments)

Subsidies (receipts) less taxes (payments) on products from/to the rest of the world

075047112599911788971259286130187012392651297394

$-\mathrm{D} .21+\mathrm{D} .31$

+ D.29-D.39

Other subsidies on production from/to the rest of the world $-\mathrm{QZPB}$

$\begin{array}{lrrrrrrr}\text { ABMI } & 1337782 & 1365685 & 1401290 & 1449861 & 1433871 & 1371163 & 1395312 \\ \text { YBGJ } & 8337 & 280 & 2495 & 2016 & -1 & -2023 & -2961\end{array}$

YBGL 1346119136596514037851451877143387013691401392351

$\begin{array}{llllllll}\text { YBGI } & 20210 & 23866 & 10066 & 22122 & 33136 & 20036 & 21984\end{array}$

$\begin{array}{llllllll}\text { QZPB } & -1383 & -4646 & -4758 & -4898 & -4906 & -4163 & -4948\end{array}$

$\begin{array}{lllllll}664 & 3716 & 3408 & 3056 & 3051 & 3351 & 2893\end{array}$

B. ${ }^{\star} \mathrm{g}$ Gross national income (GNI)

D.5,6,7 Real current transfers from the rest of the world (receipts less payments)

YBGM 1365637138892314125421472183146515113883641412280

$\begin{array}{llllllll}- \text { YBGP } & -10813 & -12054 & -11227 & -12220 & -11912 & -14037 & -17115\end{array}$

B. $6^{\star} \mathrm{g}$ Gross national disposable income

YBGO $13548241376871 \quad 14013151459964145323913743271395165$

\section{Adjustment to basic prices}

B.1* $\mathrm{g}$ Gross domestic product (at market prices)

-D.21 Adjustment to basic prices

+D.31 (less taxes plus subsidies on products)

B.1g Gross value added (at basic prices)

Net measures (after deduction of fixed

-K.1 capital consumption) at market prices

B. $5^{*} \mathrm{n} \quad$ Net national income at market prices

B.6*n Net national disposable income at market prices

ABMI $\quad 1337782136568514012901449861143387113711631395312$

$\begin{array}{lllllllll}\text {-NTAQ } & -144020 & -144884 & -149352 & -153669 & -149983 & -145769 & -149 & -142\end{array}$

ABMM 1193753122078712518741296131128386312253991246080

$\begin{array}{lllllllll}\text {-CIHA } & -138 & -187 & -139711 & -146702 & -149893 & -144846 & -150107 & -149928\end{array}$

YBET $\quad 1212271 \quad 1234697 \quad 12519391310714 \quad 1313781 \quad 1242226 \quad 1262352$ YBEY $1201458 \quad 1222647 \quad 12407101298495 \quad 130186912281891245237$

1 Capital consumption CVM estimates are still referenced to 2006. Estimates

for these series with CVM reference year 2008 will be published in the

"Capital Stocks and Capital Consumption" statistical bulletin in 2012. 
Gross domestic product: Output

B.1g Gross value added, at basic prices

P.1 Output of goods and services

-P.2 less intermediate consumption

B.1g Total Gross Value Added

D.211 Value added taxes (VAT) on products

D.212,4 Other taxes on products

-D.31 less subsidies on products

B. ${ }^{\star} \mathrm{g}$ Gross Domestic Product at market prices

\begin{tabular}{|c|c|c|c|c|c|c|c|}
\hline KN2 6 & 1509576 & 1596513 & 1684293 & 1777510 & 1860987 & 1939727 & $\begin{array}{r}204024 \\
-102554\end{array}$ \\
\hline$-\mathrm{KN} 25$ & -770134 & -814475 & -861379 & -913474 & -953594 & -982828 & -102554 \\
\hline ABML & 739442 & 782037 & 822914 & 864036 & 907393 & 956899 & 1014703 \\
\hline QYRC & 54964 & 56541 & 61512 & 64189 & 67097 & 71059 & \\
\hline NSUI & 43076 & 46999 & 50511 & 54084 & 52844 & 53946 & 548 \\
\hline -NZHC & -7469 & -6425 & -6066 & -6027 & -5709 & -6536 & -74 \\
\hline YBHA & 830013 & 879152 & 928871 & 976282 & 1021625 & 1075368 & 1139 \\
\hline
\end{tabular}

Gross domestic product: Expenditure

P.3 Final consumption expenditure

P.41 Actual individual consumption

P.3 Household final consumption expenditure

Final consumption expenditure of NPISH Individual govt. final consumption expenditure

ABPB

Total actual individual consumption

Collective govt. final consumption expenditure

Total final consumption expenditure

Households and NPISH

Central government

Local government

Gross capital formation

Gross fixed capital formation

Changes in inventories

Acquisitions less disposals of valuables

P.53

Total gross capital formation

P.6 Exports of goods and services

-P.7 less imports of goods and services

B.11 External balance of goods and services

de Statistical discrepancy between

expenditure components and GDP

B.1*g Gross Domestic Product at market prices

Gross domestic product: Income

B. $2 \mathrm{~g}$ Operating surplus, gross

Non-financial corporations

Public non-financial corporations

Private non-financial corporations

Financial corporations

General government

Households and non-profit institutions serving households

B.2g Total operating surplus, gross

B.3 Mixed income

D.1 Compensation of employees

D.2 Taxes on production and imports

-D.3 less subsidies

di Statistical discrepancy between

income components and GDP

B. ${ }^{*} \mathrm{~g}$ Gross domestic product at market prices

NQEO

NQEP

ABKW

NSSG

NMBJ

NMMT

$\begin{array}{rr}512020 & 546464 \\ 19600 & 21082\end{array}$

$90925 \quad 96432$

582295

616433

$\begin{array}{lllllll}622545 & 663978 & 708865 & 750710 & 792199 & 839074 & 885829\end{array}$

$\begin{array}{lllllll}682144 & 724274 & 774147 & 821912 & 867090 & 919627 & 974791\end{array}$

$\begin{array}{lllllll}531620 & 567546 & 604480 & 639964 & 672481 & 707071 & 742180\end{array}$

$\begin{array}{lllllll}93897 & 97156 & 103594 & 110829 & 118778 & 130348 & 142658\end{array}$

$\begin{array}{lllllll}56627 & 59572 & 66073 & 71119 & 75831 & 82208 & 89953\end{array}$

$\begin{array}{lrrrrrrr}\text { NPQX } & 138814 & 156369 & 161846 & 167063 & 171785 & 180533 & 186759\end{array}$

\begin{tabular}{|c|}
\hline NPJO \\
\hline
\end{tabular}

\begin{tabular}{lrrrrrrr} 
& & -28 & 430 & 229 & 4 & 396 & 215 \\
\cline { 2 - 7 } NQFM & 143479 & 161752 & 168121 & 172387 & 178506 & 183776 & 190965
\end{tabular}

$\begin{array}{llllllll}\text { KTMW } & 237364 & 233190 & 242614 & 269714 & 276775 & 280454 & 290207\end{array}$

\begin{tabular}{rrrrrrrr} 
KTMX & -232976 & -240062 & -256009 & -287731 & -300747 & -308488 & -316522 \\
\cline { 2 - 8 } KTMY & 4388 & -6872 & -13395 & -18017 & -23972 & -28034 & -26315
\end{tabular}

KTMY

RVFD

YBHA

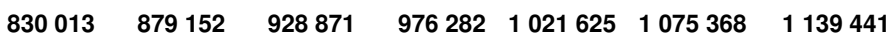

\begin{tabular}{|c|c|c|c|c|c|c|c|}
\hline NRJT & 7230 & 7751 & 7766 & 7103 & 6754 & 6631 & 7152 \\
\hline NRJK & 171667 & 174309 & 176805 & 180909 & 182932 & 187332 & 197893 \\
\hline NQNV & 21936 & 17714 & 18552 & 12016 & 13699 & 27198 & 33065 \\
\hline NMXV & 9003 & 8999 & 9262 & 9542 & 9796 & 10289 & 10807 \\
\hline QWLS & 38109 & 42229 & 45129 & 49170 & 53000 & 55647 & 6098 \\
\hline ABNF & 247945 & 251002 & 257514 & 258740 & 266181 & 287097 & 30990 \\
\hline QWLT & 47502 & 50629 & 53801 & 56807 & 60638 & 66316 & 723 \\
\hline HAEA & 429761 & 465640 & 496097 & 532315 & 564253 & 586843 & 6159 \\
\hline NZGX & 113226 & 119355 & 128527 & 135358 & 137507 & 143117 & 15066 \\
\hline AAXJ & -8419 & -7476 & -7067 & -6936 & -6953 & -8009 & -9436 \\
\hline VFC & -6 & 4 & - & 2 & - & 3 & \\
\hline 3 & 830013 & 879152 & 928871 & 976282 & 1021625 & 1075368 & 1139 \\
\hline
\end{tabular}

GROSS NATIONAL INCOME at market prices

B. $1^{\star} \mathrm{g} \quad$ Gross Domestic Product at market prices

Compensation of employees

receipts from the rest of the world (ROW)

less payments to the rest of the world (ROW)

YBHA

$\begin{array}{lllllllll}830 & 013 & 879152 & 928871 & 976282 & 1021625 & 1075368 & 1139441\end{array}$

KTMN

KTMO

D.1 Total

KTMP

\begin{tabular}{rrrrrrr}
1007 & 840 & 960 & 1032 & 1087 & 1121 & 1116 \\
-924 & -850 & -759 & -882 & -1021 & -1054 & -1057 \\
\hline 83 & -10 & 201 & 150 & 66 & 67 & 59
\end{tabular}

less Taxes on products paid to the ROW

Other subsidies on production

D.4 Property and entrepreneurial income receipts from the rest of the world less payments to the rest of the world

HMBN

$\begin{array}{rrrrrrr}93360 & 102548 & 100733 & 131902 & 137447 & 120543 & 122069\end{array}$

HMBO 
GROSS DOMESTIC PRODUCT

$2004 \quad 2005$

2006

2007

2008

2009

Gross domestic product: Output

B.1g Gross value added, at basic prices

P.1 Output of goods and services ${ }^{1}$

-P.2 less intermediate consumption ${ }^{1}$

B.1g Total Gross Value Added

D.211 Value added taxes (VAT) on products

D.212,4 Other taxes on products

-D.31 less subsidies on products

B. ${ }^{\star} \mathrm{g} \quad$ Gross Domestic Product at market prices

$\begin{array}{llllllll}\text { KN2 } 6 & 2140984 & 2258356 & 2398550 & 2537820 & 2630445 & 2590111\end{array}$

-KN25 -1070614 -1141474 -1214609 -1285218 -1346562 -1333179

$\begin{array}{llllllll}A B M L & 1070369 & 1116882 & 1183940 & 1252602 & 1283884 & 1256932 & 1301118\end{array}$

$\begin{array}{lrrrrrrr}\text { QYRC } & 81544 & 83425 & 87758 & 92017 & 91952 & 79900 & 95964\end{array}$

$\begin{array}{llllllll}\text { NSUI } & 58308 & 59167 & 62865 & 66786 & 63186 & 62729 & 67934\end{array}$

$\begin{array}{llllllll}- \text { NZHC } & -7851 & -5182 & -5966 & -5609 & -5152 & -5707 & -6564\end{array}$

$\begin{array}{lllllllll}\text { YBHA } & 1202370 & 1254292 & 1328597 & 1405796 & 1433870 & 1393854 & 1458452\end{array}$

Gross domestic product: Expenditure

P.3 Final consumption expenditure

P.41 Actual individual consumption

P.3 Household final consumption expenc

Final consumption expenditure of NPISH

Individual govt. final consumption expenditure

Total actual individual consumption

Collective govt. final consumption expenditure

Total final consumption expenditure

Households and NPISH

Central government

Local government

Gross capital formation

Gross fixed capital formation

Changes in inventories

Acquisitions less disposals of valuables

P.5 Total gross capital formation

P.6 Exports of goods and services

-P.7 less imports of goods and services

B.11 External balance of goods and services

de Statistical discrepancy between

expenditure components and GDP

B.1*g Gross Domestic Product at market prices

\begin{tabular}{|c|c|c|c|c|c|c|c|}
\hline $\begin{array}{l}\text { ABPB } \\
\text { ABNV } \\
\text { NNAQ }\end{array}$ & $\begin{array}{r}749607 \\
29197 \\
147751\end{array}$ & $\begin{array}{r}784149 \\
30824 \\
159195\end{array}$ & $\begin{array}{r}819164 \\
32408 \\
172489\end{array}$ & $\begin{array}{r}862242 \\
34324 \\
181762\end{array}$ & $\begin{array}{r}878024 \\
35767 \\
194621\end{array}$ & $\begin{array}{r}858242 \\
35863 \\
206913\end{array}$ & $\begin{array}{r}900204 \\
37702 \\
212764\end{array}$ \\
\hline NQEO & 926555 & 974168 & 1024061 & 1078328 & 1108412 & 1101018 & 1150670 \\
\hline NQEP & 103177 & 109078 & 112637 & 113392 & 120945 & 120436 & 125303 \\
\hline $\begin{array}{l}\text { ABKW } \\
\text { NSSG } \\
\text { NMBJ } \\
\text { NMMT }\end{array}$ & $\begin{array}{r}1029732 \\
778804 \\
152274 \\
98654\end{array}$ & $\begin{array}{r}1083246 \\
814973 \\
161329 \\
106944\end{array}$ & $\begin{array}{r}1136698 \\
851572 \\
173416 \\
111710\end{array}$ & $\begin{array}{r}1191720 \\
896566 \\
178058 \\
117096\end{array}$ & $\begin{array}{r}1229357 \\
913791 \\
191348 \\
124218\end{array}$ & $\begin{array}{r}1221454 \\
894105 \\
199649 \\
127700\end{array}$ & $\begin{array}{r}1275973 \\
937906 \\
207349 \\
130718\end{array}$ \\
\hline $\begin{array}{l}\text { NPQX } \\
\text { ABMP } \\
\text { NPJO }\end{array}$ & $\begin{array}{r}200430 \\
4890 \\
-36\end{array}$ & $\begin{array}{r}209722 \\
4405 \\
-377\end{array}$ & $\begin{array}{r}227172 \\
5172 \\
285\end{array}$ & $\begin{array}{r}250036 \\
6224 \\
465\end{array}$ & $\begin{array}{r}241364 \\
1711 \\
561\end{array}$ & $\begin{array}{r}209253 \\
-11651 \\
429\end{array}$ & $\begin{array}{r}217108 \\
6832 \\
638\end{array}$ \\
\hline NQFM & 205285 & 213750 & 232630 & 256725 & 243634 & 198035 & 224577 \\
\hline $\begin{array}{r}\text { KTMW } \\
\text { - KTMX }\end{array}$ & $\begin{array}{r}303612 \\
-336255\end{array}$ & $\begin{array}{r}331067 \\
-373771\end{array}$ & $\begin{array}{r}379091 \\
-419822\end{array}$ & $\begin{array}{r}374032 \\
-416681\end{array}$ & $\begin{array}{r}422864 \\
-461988\end{array}$ & $\begin{array}{r}395588 \\
-421225\end{array}$ & $\begin{array}{r}436796 \\
-476480\end{array}$ \\
\hline KTMY & -32643 & -42704 & -40731 & -42649 & -39124 & -25637 & -39684 \\
\hline RVFD & -1 & - & -1 & - & 1 & - & -2415 \\
\hline YBHA & 1202370 & 1254292 & 1328597 & 1405796 & 1433870 & 1393854 & 1458452 \\
\hline
\end{tabular}

Gross domestic product: Income

B. $2 \mathrm{~g}$

erating surplus, gros

Non-financial corporations

Public non-financial corporations

Private non-financial corporations

Financial corporations

General government

Households and non-profit institutions serving households

Total operating surplus, gross

B.2g Mixed income

D.1 Compensation of employees

D.2 Taxes on production and imports

-D.3 less subsidies

di Statistical discrepancy between

income components and GDP

B. ${ }^{\star} \mathrm{g}$ Gross domestic product at market prices

\begin{tabular}{|c|c|c|c|c|c|c|c|}
\hline NRJT & 6860 & 8473 & 9628 & 10114 & 8043 & 9496 & 8810 \\
\hline NRJK & 216750 & 224811 & 244309 & 257995 & 255260 & 233436 & 246074 \\
\hline NQNV & 33654 & 34323 & 39098 & 43643 & 57758 & 65816 & 52898 \\
\hline NMXV & 11312 & 11927 & 12634 & 13231 & 13963 & 14675 & 15500 \\
\hline QWLS & 65752 & 67494 & 69807 & 77768 & 74878 & 57744 & 7209 \\
\hline ABNF & 334328 & 347028 & 375476 & 402751 & 409902 & 381167 & 39538 \\
\hline QWLT & 73335 & 78487 & 80432 & 82898 & 86376 & 81424 & 8104 \\
\hline HAEA & 646006 & 677517 & 713513 & 751858 & 770969 & 776872 & 799974 \\
\hline NZGX & 158704 & 162298 & 171454 & 180335 & 178207 & 166823 & 191829 \\
\hline -AAXJ & -10005 & -11039 & -12280 & -12047 & -11584 & -12431 & -12819 \\
\hline RVFC & 3 & -2 & -2 & 2 & 1 & -8 & 3041 \\
\hline YBHA & 1202370 & 1254292 & | 328597 & 1405796 & 1433870 & 1393854 & 458 \\
\hline
\end{tabular}

GROSS NATIONAL INCOME at market prices

B. ${ }^{\star} \mathrm{g}$ Gross Domestic Product at market prices

D.1 Compensation of employees

receipts from the rest of the world (ROW) less payments to the rest of the world (ROW)

D.1 Total

\begin{tabular}{|c|c|c|c|c|c|c|c|}
\hline YBHA & 1202370 & 1254292 & 1328597 & 1405796 & 1433870 & 1393854 & 1458452 \\
\hline KTMN & 931 & 974 & 938 & 984 & 1046 & 1176 & 1097 \\
\hline -Ктмо & -1425 & -1584 & -1896 & -1718 & -1761 & -1435 & -1486 \\
\hline KTMP & -494 & -610 & -958 & -734 & -715 & -259 & -389 \\
\hline -QZOZ & -1234 & -4260 & -4496 & -4731 & -4906 & -4238 & -5186 \\
\hline - IBJL & 592 & 3408 & 3221 & 2952 & 3051 & 3411 & 3032 \\
\hline HMBN & 137380 & 185640 & 237505 & 291614 & 262 & 169313 & 162366 \\
\hline - HMBO & -118857 & -163144 & -227035 & -269511 & -228991 & -148657 & -138938 \\
\hline HMBM & 18523 & 22496 & 10470 & 22103 & 33851 & 20656 & 23428 \\
\hline ABMX & 1219759 & 1275323 & 1336830 & 1425387 & 1465152 & 1413417 & 1479337 \\
\hline
\end{tabular}

B.5 ${ }^{\star} \mathrm{g}$ Gross National Income at market prices

less Taxes on products paid to the ROW plus Subsidies received from the ROW

+D.29-D.39 Other subsidies on production

D.4 Property and entrepreneurial income receipts from the rest of the world less payments to the rest of the world

D.4 Total

1 These series are not available for the latest year 
GROSS DOMESTIC PRODUCT

1997

1998

1999

2000

2001

2002

2003

Gross domestic product: expenditure approach

Final consumption expenditure

Actual individual consumption

Household final consumption expenditure

Final consumption expenditure of non-profit institutions serving households

Individual government final consumption expenditure

Total actual individual consumption

Collective government final consumption expenditure

P.3 Total final consumption expenditure

Gross capital formation

Gross fixed capital formation

Changes in inventories

Acquisitions less disposals of valuables

Total gross capital formation

Gross domestic final expenditure

P.6 Exports of goods and services

Gross final expenditure

-P.7 less imports of goods and services

de Statistical discrepancy between

expenditure components and GDP

B.1* $\mathrm{g}$ Gross domestic product at market prices

\begin{tabular}{|c|c|c|c|c|c|c|c|}
\hline ABPF & 616065 & 645651 & 680852 & 718644 & 748122 & 781860 & 807653 \\
\hline ABNU & 33851 & 35635 & 35944 & 36479 & 37037 & 36615 & 36266 \\
\hline NSZK & 1534 & 156 & 159 & 162 & 166 & 172 & 176681 \\
\hline YBIO & 798100 & 832937 & 8731 & & 950375 & & 1020298 \\
\hline NSZL & & & 90 & & & 10 & 111027 \\
\hline$A B K X$ & 882203 & 918691 & 964196 & 1012225 & 1049625 & 1093862 & 1131451 \\
\hline NPQR & 159082 & 180 & 185 & 190 & 195 & 202 & 204883 \\
\hline ABMQ & 7281 & 980 & 6393 & 6047 & 7989 & 1862 & 5044 \\
\hline NPJP & -148 & 550 & 274 & 4 & 457 & 236 & -42 \\
\hline NPQU & 162445 & 182925 & 188018 & 192052 & 199328 & 200670 & 208248 \\
\hline YBIK & 1043490 & 1102010 & 1152231 & 1203797 & 1248470 & 1293540 & 1338724 \\
\hline KTMZ & 265377 & 275661 & 285142 & 312442 & 322079 & 328327 & 334505 \\
\hline ABME & 1310420 & 1378839 & 1438466 & 1517859 & 1572158 & 1623417 & 1674822 \\
\hline -KTNB & -257876 & -283985 & -303243 & -331623 & -348298 & -366783 & -373815 \\
\hline GIXS & - & -1 & - & - & - & - & - \\
\hline ABMI & 1054232 & 1094704 & 1134723 & 1185305 & 1222650 & 1255142 & 1299381 \\
\hline
\end{tabular}

B.11 of which External balance of goods and services

KTNC

7501

$\begin{array}{lllll}-8324 & -18101 & -19181 & -26219 & -38456\end{array}$

$-39310$ 


\section{UK gross domestic product}

Chained volume measures (reference year 2008) continued

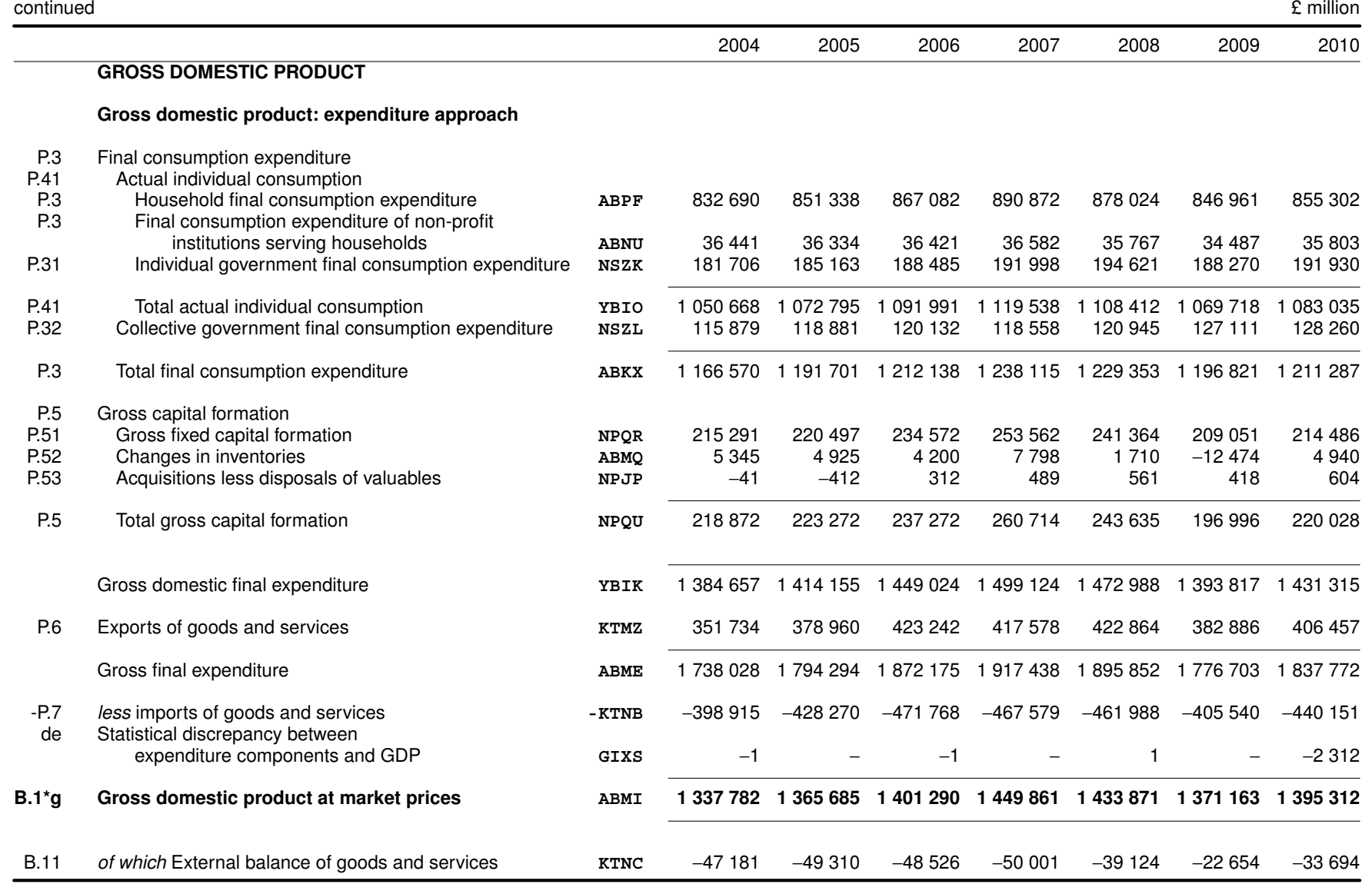


Indices $2008=100$

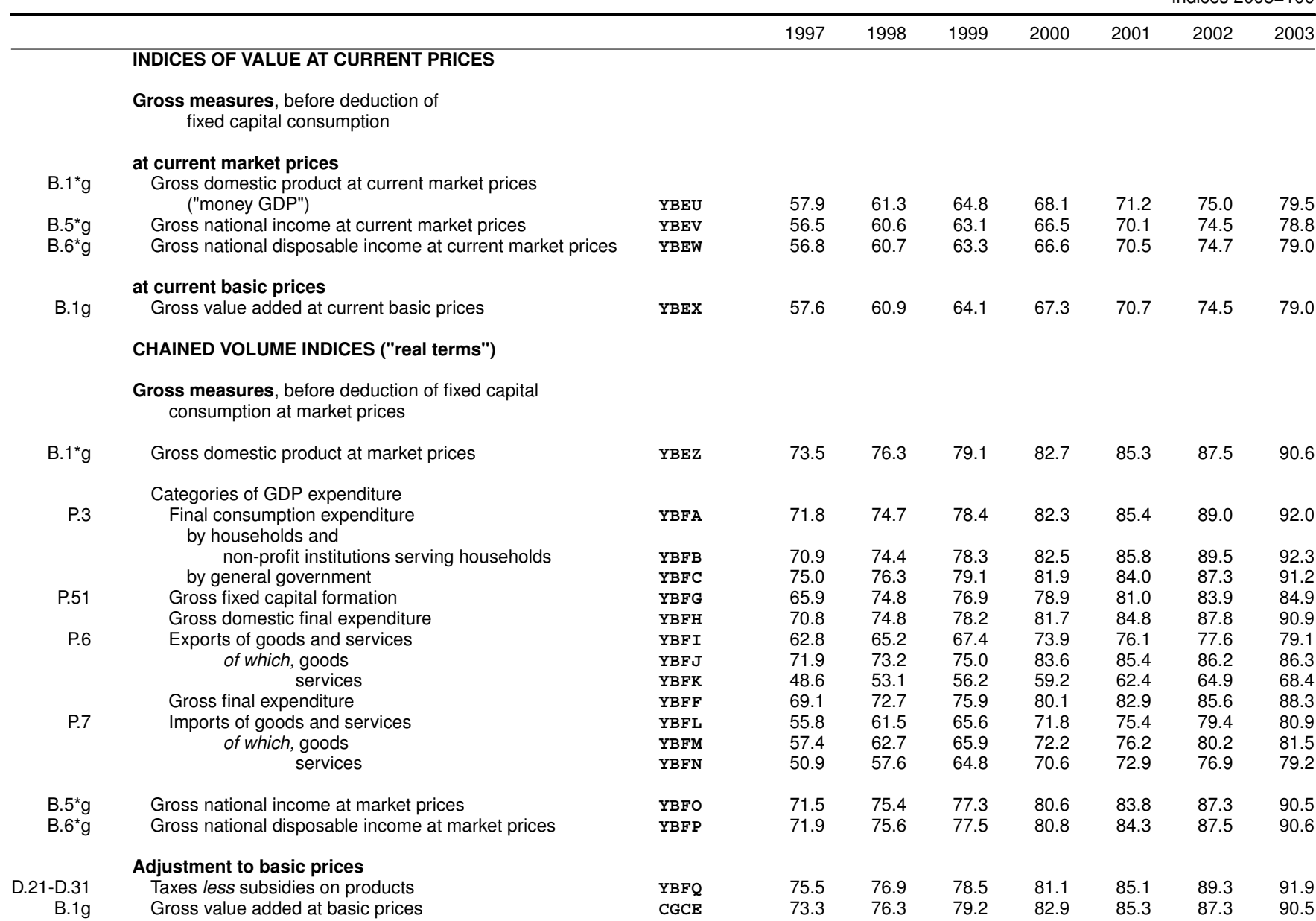

PRICE INDICES (IMPLIED DEFLATORS) ${ }^{1}$

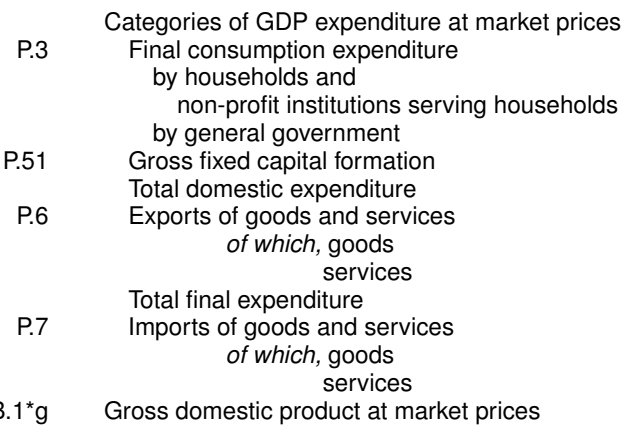

\begin{tabular}{llllllll} 
YBGA & 77.3 & 78.8 & 80.3 & 81.2 & 82.6 & 84.1 & 86.2 \\
& & & & & & & \\
YBFS & 82.0 & 83.5 & 84.5 & 84.9 & 85.8 & 86.4 & 88.0 \\
YBFT & 63.6 & 65.1 & 67.9 & 70.4 & 73.4 & 77.1 & 80.8 \\
YBFU & 87.3 & 86.6 & 87.2 & 87.7 & 87.9 & 89.1 & 91.2 \\
YBFV & 79.1 & 80.4 & 81.8 & 82.6 & 83.8 & 85.3 & 87.1 \\
YBFW & 89.4 & 84.6 & 85.1 & 86.3 & 86.0 & 85.4 & 86.8 \\
BQNK & 94.8 & 88.8 & 87.9 & 89.1 & 87.8 & 85.8 & 86.5 \\
FKNW & 78.8 & 76.3 & 79.6 & 80.8 & 82.3 & 84.7 & 87.2 \\
YBFY & 81.1 & 81.2 & 82.4 & 83.3 & 84.1 & 85.2 & 86.9 \\
YBFZ & 90.3 & 84.5 & 84.4 & 86.8 & 86.4 & 84.1 & 84.7 \\
BQNL & 92.7 & 85.6 & 85.5 & 88.4 & 87.2 & 84.3 & 83.9 \\
FHMA & 82.8 & 81.3 & 81.2 & 81.6 & 83.5 & 83.4 & 86.8 \\
YBGB & 78.7 & 80.3 & 81.9 & 82.4 & 83.6 & 85.7 & 87.7 \\
\hline
\end{tabular}

\section{HOME COSTS PER UNIT OF OUTPUT ${ }^{2}$}

\begin{tabular}{|c|c|c|c|c|c|c|c|c|c|}
\hline B. $1^{*} \mathrm{~g}$ & $\begin{array}{l}\text { Total home costs (based on expenditure } \\
\text { components of GDP) }\end{array}$ & YBGC & 78.0 & 79.3 & 80.4 & 80.7 & 82.4 & 84.9 & 87.0 \\
\hline & Compensation of employees & YBGD & 75.8 & 79.1 & 81.3 & 83.5 & 85.8 & 87.0 & 88.2 \\
\hline B. $2 g, B .3 g$ & Gross operating surplus and mixed income & YBGE & 81.0 & 79.6 & 79.3 & 76.9 & 77.2 & 81.4 & 85.0 \\
\hline
\end{tabular}

\footnotetext{
1 Implied deflators are derived by dividing the estimates for each component

at current market prices by the corresponding chained volume estimate.

2 These index numbers show how employment and operating incomes relate

to the implied deflator of GDP at market prices.
} 


$\begin{array}{lllllll}2004 & 2005 & 2006 & 2007 & 2008 & 2009 & 2010\end{array}$

INDICES OF VALUE AT CURRENT PRICES

Gross measures, before deduction of

fixed capital consumption

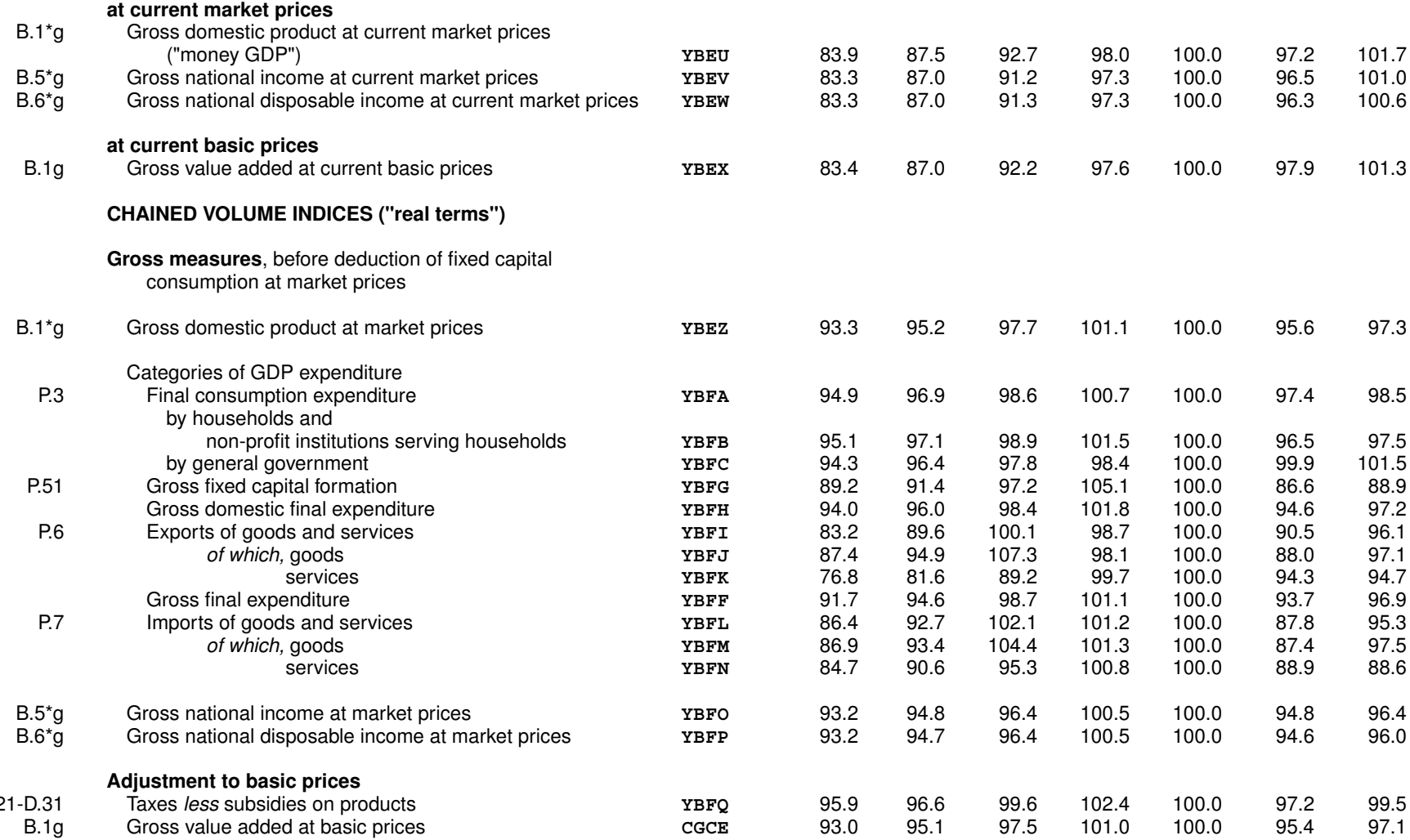

\section{PRICE INDICES (IMPLIED DEFLATORS) ${ }^{1}$}

Categories of GDP expenditure at market prices

Final consumption expenditure

by households and

non-profit institutions serving households

by general government

P.51 Gross fixed capital formation

Total domestic expenditure

P.6 Exports of goods and services of which, goods

Total final expenditure

$$
\text { services }
$$

P.7 Imports of goods and services

$$
\text { of which, goods }
$$

B. $1^{*} \mathrm{~g} \quad$ Gross domestic product at market prices

\begin{tabular}{llllllll} 
YBGA & 88.3 & 90.9 & 93.8 & 96.3 & 100.0 & 102.1 & 105.3 \\
& & & & & & & \\
YBFS & 89.6 & 91.8 & 94.3 & 96.7 & 100.0 & 101.4 & 105.3 \\
YBFT & 84.3 & 88.2 & 92.4 & 95.0 & 100.0 & 103.8 & 105.6 \\
YBFU & 93.1 & 95.1 & 96.8 & 98.6 & 100.0 & 100.1 & 101.2 \\
YBFV & 89.2 & 91.7 & 94.5 & 96.6 & 100.0 & 101.8 & 104.8 \\
YBFW & 86.3 & 87.4 & 89.6 & 89.6 & 100.0 & 103.3 & 107.5 \\
BQNK & 86.6 & 88.4 & 90.0 & 89.1 & 100.0 & 102.9 & 108.7 \\
FKNW & 85.9 & 85.8 & 88.9 & 90.2 & 100.0 & 103.9 & 105.7 \\
YBFY & 88.5 & 90.7 & 93.4 & 95.0 & 100.0 & 102.2 & 105.4 \\
YBFZ & 84.3 & 87.3 & 89.0 & 89.1 & 100.0 & 103.9 & 108.3 \\
BQNL & 83.7 & 86.7 & 88.5 & 88.6 & 100.0 & 102.8 & 107.9 \\
FHMA & 86.1 & 89.0 & 90.2 & 90.5 & 100.0 & 107.0 & 109.3 \\
YBGB & 89.9 & 91.8 & 94.8 & 97.0 & 100.0 & 101.7 & 104.5 \\
\hline
\end{tabular}

\begin{tabular}{|c|c|c|c|c|c|c|c|c|c|}
\hline B. $1^{*} \mathrm{~g}$ & $\begin{array}{l}\text { Total home costs (based on expenditure } \\
\text { components of GDP) }\end{array}$ & YBGC & 89.4 & 91.5 & 94.6 & 96.7 & 100.0 & 102.6 & 104.0 \\
\hline D. 1 & Compensation of employees & YBGD & 89.8 & 92.3 & 94.7 & 96.4 & 100.0 & 105.4 & 106.6 \\
\hline B. $2 g, B .3 g$ & Gross operating surplus and mixed income & YBGE & 88.0 & 90.0 & 94.0 & 96.8 & 100.0 & 97.5 & 98.7 \\
\hline
\end{tabular}

\section{HOME COSTS PER UNIT OF OUTPUT ${ }^{2}$}

1 Implied deflators are derived by dividing the estimates for each component

at current market prices by the corresponding chained volume estimate.

2 These index numbers show how employment and operating incomes relate

to the implied deflator of GDP at market prices. 


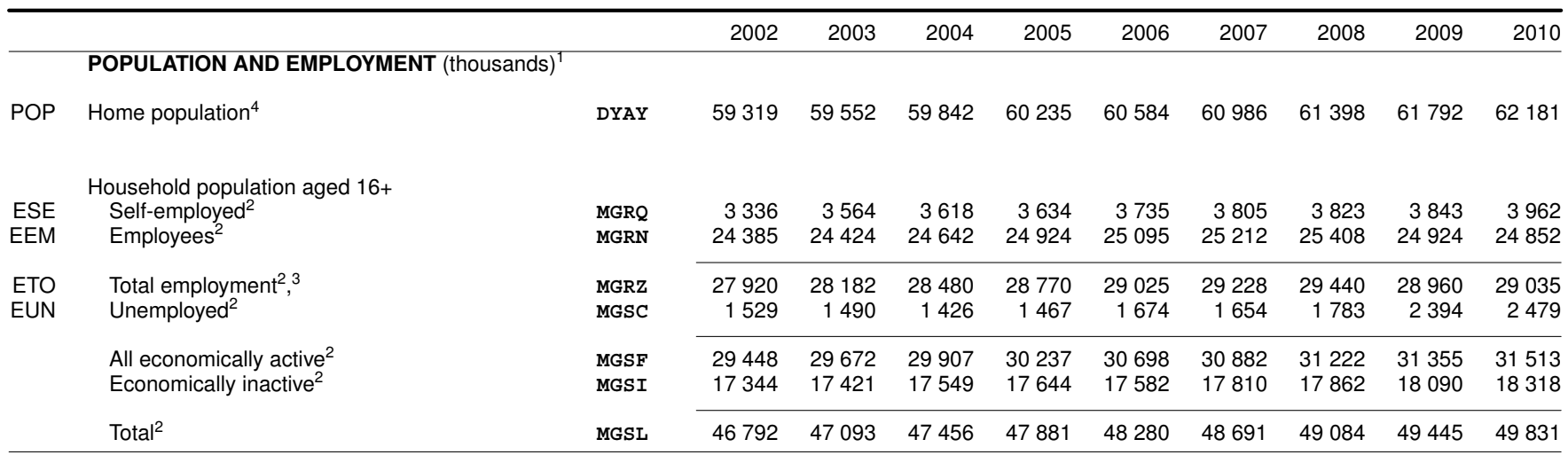

\section{GROSS DOMESTIC PRODUCT PER HEAD $£$}

At current prices

Gross domestic product at market prices ${ }^{4}$

Chained volume measures

market prices

IHXT

$18127 \quad 19131 \quad 20089$

20822

$21929 \quad 23050 \quad 23354$

22556

23527

Gross value added at basic prices

IHXW

$\begin{array}{lll}21 & 158 & 21817 \\ 18 & 354\end{array}$

22671

$23130 \quad 23774 \quad 23354$

$22190 \quad 22509$ YBGT

18900

9503

19947

$20663 \quad 21253$

1 Components may not sum to totals due to rounding.

2 These seasonally adjusted data are 4 quarter annual averages derived

from quarterly Labour Force Survey, which does not include those resident in communal establishments except for those in student halls of residence and NHS accommodation.

3 Includes people on Government-supported training and employment programmes and unpaid family workers.

4 These data are consistent with the population estimates published on 23 June 2011 


\begin{tabular}{|c|c|c|c|c|c|c|c|c|c|c|c|}
\hline & & & 2002 & 2003 & 2004 & 2005 & 2006 & 2007 & 2008 & 2009 & 2010 \\
\hline \multirow[t]{2}{*}{0} & GOODS AND SERVICES ACCOUNT & & & & & & & & & & \\
\hline & $\begin{array}{l}\text { Resources } \\
\text { Output }\end{array}$ & & & & & & & & & & \\
\hline P.11 & Market output ${ }^{1}$ & NQAG & 1620121 & 1691699 & 1768437 & 1861448 & 1977972 & 2098062 & 2162750 & 2107552 & .. \\
\hline P.12 & Output for own final use ${ }^{1}$ & $\mathrm{NQAH}$ & 80628 & 88271 & 92422 & 97811 & 103044 & 110280 & 116362 & 119347 & .. \\
\hline P.13 & Other non-market output ${ }^{1}$ & NQAI & 238978 & 260279 & 280125 & 299097 & 317534 & 329478 & 351333 & 363212 & .. \\
\hline P.1 & Total output $^{1}$ & $\mathrm{KN} 26$ & 1939727 & 2040249 & 2140984 & 2258356 & 2398550 & 2537820 & 2630445 & 2590111 & .. \\
\hline D.21 & Taxes on products & NZGW & 125004 & 132148 & 139851 & 142592 & 150623 & 158804 & 155138 & 142629 & 163898 \\
\hline -D.31 & less Subsidies on products & $-\mathrm{NZHC}$ & -6536 & -7410 & -7851 & -5182 & -5966 & -5609 & -5152 & -5707 & -6564 \\
\hline P.7 & Imports of goods and services & KTMX & 308488 & 316522 & 336255 & 373771 & 419822 & 416681 & 461988 & 421225 & 476480 \\
\hline \multirow[t]{2}{*}{ Total } & Total resources ${ }^{1}$ & NQBM & 2366683 & 2481509 & 2609239 & 2769537 & 2963029 & 3107696 & 3242419 & 3148258 & .. \\
\hline & Uses & & & & & & & & & & \\
\hline P.2 & Intermediate consumption ${ }^{1}$ & KN25 & 982828 & 1025546 & 1070614 & 1141474 & . 1214609 & 1285218 & 1346562 & 1333179 & .. \\
\hline P.3 & Final consumption expenditure & & & & & & & & & & \\
\hline $\begin{array}{l}\text { P.31 } \\
\text { P.31 }\end{array}$ & $\begin{array}{l}\text { By households } \\
\text { By non-profit institutions }\end{array}$ & $\mathrm{ABPB}$ & 680649 & 714512 & 749607 & 784149 & 819164 & 862242 & 878024 & 858242 & 900204 \\
\hline & serving households & ABNV & 26422 & 27668 & 29197 & 30824 & 32408 & 34324 & 35767 & 35863 & 37702 \\
\hline P.3 & By government & & & & & & & & & & \\
\hline P.31 & For individual consumption & NNAQ & 132003 & 143649 & 147751 & 159195 & 172489 & 181762 & 194621 & 206913 & 212764 \\
\hline P.32 & For collective consumption & NQEP & 80553 & 88962 & 103177 & 109078 & 112637 & 113392 & 120945 & 120436 & 125303 \\
\hline P.3 & Total by government & NMRK & 212556 & 232611 & 250928 & 268273 & 285126 & 295154 & 315566 & 327349 & 338067 \\
\hline P.3 & Total final consumption expenditure ${ }^{2}$ & ABKW & 919627 & 974791 & 1029732 & 1083246 & 1136698 & 1191720 & 1229357 & 1221454 & 1275973 \\
\hline P.5 & Gross capital formation & & & & & & & & & & \\
\hline P.51 & Gross fixed capital formation & NPQX & 180533 & 186759 & 200430 & 209722 & 227172 & 250036 & 241364 & 209253 & 217108 \\
\hline P.52 & Changes in inventories & ABMP & 3026 & 4242 & 4890 & 4405 & 5172 & 6224 & 1711 & -11651 & 6832 \\
\hline P.53 & Acquisitions less disposals of valuables & NPJO & 215 & -37 & -36 & -377 & 285 & 465 & 561 & 429 & 638 \\
\hline P.5 & Total gross capital formation & NQFM & 183776 & 190965 & 205285 & 213750 & 232630 & 256725 & 243634 & 198035 & 224577 \\
\hline P.6 & Exports of goods and services & KTMW & 280454 & 290207 & 303612 & 331067 & 379091 & 374032 & 422864 & 395588 & 436796 \\
\hline \multirow[t]{2}{*}{ de } & Statistical discrepancy between & & & & & & & & & & \\
\hline & expenditure components and GDP & RVFD & - & - & -1 & - & -1 & - & 1 & - & -2415 \\
\hline Total & Total uses ${ }^{1}$ & NQBM & 2366683 & 2481509 & 2609239 & 2769537 & 2963029 & 3107696 & 3242419 & 3148258 & .. \\
\hline
\end{tabular}

1 These series are not available for the latest year

2 For the total economy, total final consumption expenditure $=$ P.4 actual final consumption 


\begin{tabular}{|c|c|c|c|c|c|c|c|c|c|c|}
\hline & & & 2002 & 2003 & 2004 & 2005 & 2006 & 2007 & 2008 & 2009 \\
\hline \multirow[t]{2}{*}{$I$} & PRODUCTION ACCOUNT & & & & & & & & & \\
\hline & Resources & & & & & & & & & \\
\hline P.1 & Output & & & & & & & & & \\
\hline P.11 & Market output & NQAG & 1620121 & 1691699 & 1768437 & 1861448 & 1977972 & 2098062 & 2162750 & 2107552 \\
\hline P.12 & Output for own final use & NQAH & 80628 & 88271 & 92422 & 97811 & 103044 & 110280 & 116362 & 119347 \\
\hline P.13 & Other non-market output & NQAI & 238978 & 260279 & 280125 & 299097 & 317534 & 329478 & 351333 & 363212 \\
\hline D.21 & Taxes on products & NZGW & 125004 & 132148 & 139851 & 142592 & 150623 & 158804 & 155138 & 142629 \\
\hline$-D .31$ & less Subsidies on products & $-\mathrm{NZHC}$ & -6536 & -7410 & -7851 & -5182 & -5966 & -5609 & -5152 & -5707 \\
\hline Total & Total resources & NQBP & 2058196 & 2164987 & 2272985 & 2395766 & 2543207 & 2691014 & 2780431 & 2727033 \\
\hline
\end{tabular}

\begin{tabular}{|c|c|c|c|c|c|c|c|c|c|c|}
\hline $\begin{array}{r}\text { P. } 2 \\
\text { B. } \mathbf{1}^{*} \mathbf{g}\end{array}$ & $\begin{array}{l}\text { Uses } \\
\text { Intermediate consumption } \\
\text { Gross domestic product }\end{array}$ & $\begin{array}{l}\text { KN25 } \\
\text { YBHA }\end{array}$ & $\begin{array}{r}982828 \\
1075368\end{array}$ & $\begin{array}{l}1025546 \\
1139441\end{array}$ & $\begin{array}{l}1070614 \\
1202370\end{array}$ & $\begin{array}{l}1141474 \\
1254292\end{array}$ & $\begin{array}{l}1214609 \\
1328597\end{array}$ & $\begin{array}{l}1285218 \\
1405796\end{array}$ & $\begin{array}{l}1346562 \\
1433870\end{array}$ & $\begin{array}{l}1333179 \\
1393854\end{array}$ \\
\hline Total & Total uses & NQBP & 2058196 & 2164987 & 2272985 & 2395766 & 2543207 & 2691014 & 2780431 & 2727033 \\
\hline $\begin{aligned} \text { B. } \mathbf{1}^{*} \mathbf{g} \\
-\mathrm{K} .1\end{aligned}$ & $\begin{array}{l}\text { Gross domestic product } \\
\text { less Fixed capital consumption }\end{array}$ & $\begin{array}{r}\text { YBHA } \\
\text {-NQAE }\end{array}$ & $\begin{array}{r}1075368 \\
-121914\end{array}$ & $\begin{array}{r}1139441 \\
-125603\end{array}$ & $\begin{array}{r}1202370 \\
-135067\end{array}$ & $\begin{array}{r}1254292 \\
-138272\end{array}$ & $\begin{array}{r}1328597 \\
-147323\end{array}$ & $\begin{array}{r}1405796 \\
-154297\end{array}$ & $\begin{array}{r}1433870 \\
-151370\end{array}$ & $\begin{array}{r}1393854 \\
-159862\end{array}$ \\
\hline
\end{tabular}

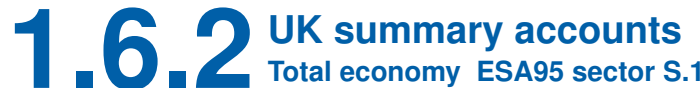

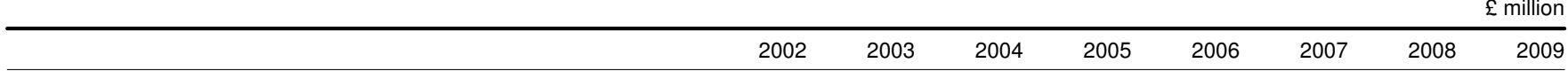

II DISTRIBUTION AND USE OF INCOME ACCOUNTS

II.1 PRIMARY DISTRIBUTION OF INCOME ACCOUNT

II.1.1 GENERATION OF INCOME ACCOUNT

Resources

B.1* $\mathrm{T}$ Total resources (gross domestic product)

Uses

D.1 Compensation of employees

D.11 Wages and salaries

D.12 Employers' social contributions

D.1 Total

D.2 Taxes on production and imports, paid

D.21 Taxes on products and imports

D.29 Production taxes other than on products

D.2 Total taxes on production and imports

-D.3 less Subsidies, received

-D.31 Subsidies on products

-D.39 Production subsidies other than on products

-D.3 Total subsidies on production

B.2g Operating surplus, gross

B.3g Mixed income, gross

di Statistical discrepancy between

income components and GDP

B.1 ${ }^{\star} \mathrm{g}$ Total uses (gross domestic product)

-K.1 After deduction of fixed capital consumption:

B.2n Operating surplus, net

B.3n Mixed income, net
YBHA 10753681139441120237012542921328597140579614338701393854

\begin{tabular}{|c|c|c|c|c|c|c|c|c|}
\hline $\begin{array}{l}\text { NQAU } \\
\text { NQAV }\end{array}$ & $\begin{array}{r}508704 \\
78139\end{array}$ & $\begin{array}{r}526755 \\
89240\end{array}$ & $\begin{array}{r}549369 \\
96637\end{array}$ & $\begin{array}{l}570242 \\
107275\end{array}$ & $\begin{array}{l}598506 \\
115007\end{array}$ & $\begin{array}{l}632856 \\
119002\end{array}$ & $\begin{array}{l}650573 \\
120396\end{array}$ & $\begin{array}{l}64916 \\
12770\end{array}$ \\
\hline HAEA & 586843 & 615995 & 646006 & 677517 & 713513 & 751858 & 770969 & 776 \\
\hline $\begin{array}{l}\text { QZPQ } \\
\text { NMYD }\end{array}$ & $\begin{array}{r}125004 \\
18113\end{array}$ & $\begin{array}{r}132148 \\
18517\end{array}$ & $\begin{array}{r}139851 \\
18853\end{array}$ & $\begin{array}{r}142592 \\
19706\end{array}$ & $\begin{array}{r}150627 \\
20831\end{array}$ & $\begin{array}{r}158804 \\
21532\end{array}$ & $\begin{array}{r}155140 \\
23069\end{array}$ & \\
\hline NZGX & 143117 & 150665 & 158704 & 162298 & 171454 & 180335 & 178207 & 166 \\
\hline $\begin{array}{l}\text {-NZHC } \\
\text {-LIUB }\end{array}$ & $\begin{array}{l}-6536 \\
-1473\end{array}$ & $\begin{array}{l}-7410 \\
-2026\end{array}$ & $\begin{array}{l}-7851 \\
-2154\end{array}$ & $\begin{array}{l}-5182 \\
-5857\end{array}$ & $\begin{array}{l}-5966 \\
-6312\end{array}$ & $\begin{array}{l}-5609 \\
-6438\end{array}$ & $\begin{array}{l}-5152 \\
-6432\end{array}$ & $\begin{array}{l}-5 \\
-6\end{array}$ \\
\hline -AAXJ & -8009 & -9436 & -10005 & -11039 & -12280 & -12047 & -11584 & -12 \\
\hline $\begin{array}{l}\text { ABNF } \\
\text { QWLT }\end{array}$ & $\begin{array}{r}287097 \\
66316\end{array}$ & $\begin{array}{r}309900 \\
72316\end{array}$ & $\begin{array}{r}334328 \\
73335\end{array}$ & $\begin{array}{r}347028 \\
78487\end{array}$ & $\begin{array}{r}375476 \\
80432\end{array}$ & $\begin{array}{r}402751 \\
82898\end{array}$ & $\begin{array}{r}409902 \\
86376\end{array}$ & $\begin{array}{r}381 \\
81\end{array}$ \\
\hline RVFC & 3 & 1 & 3 & -2 & -2 & 2 & 1 & \\
\hline
\end{tabular}

YBHA 10753681139441120237012542921328597140579614338701393854

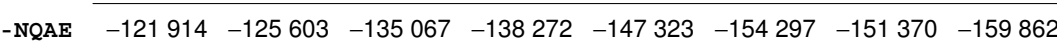

$\begin{array}{lllllllll}\text { NOAR } & 180754 & 199848 & 219429 & 228575 & 251926 & 274400 & 276148 & 240674\end{array}$

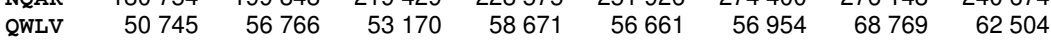




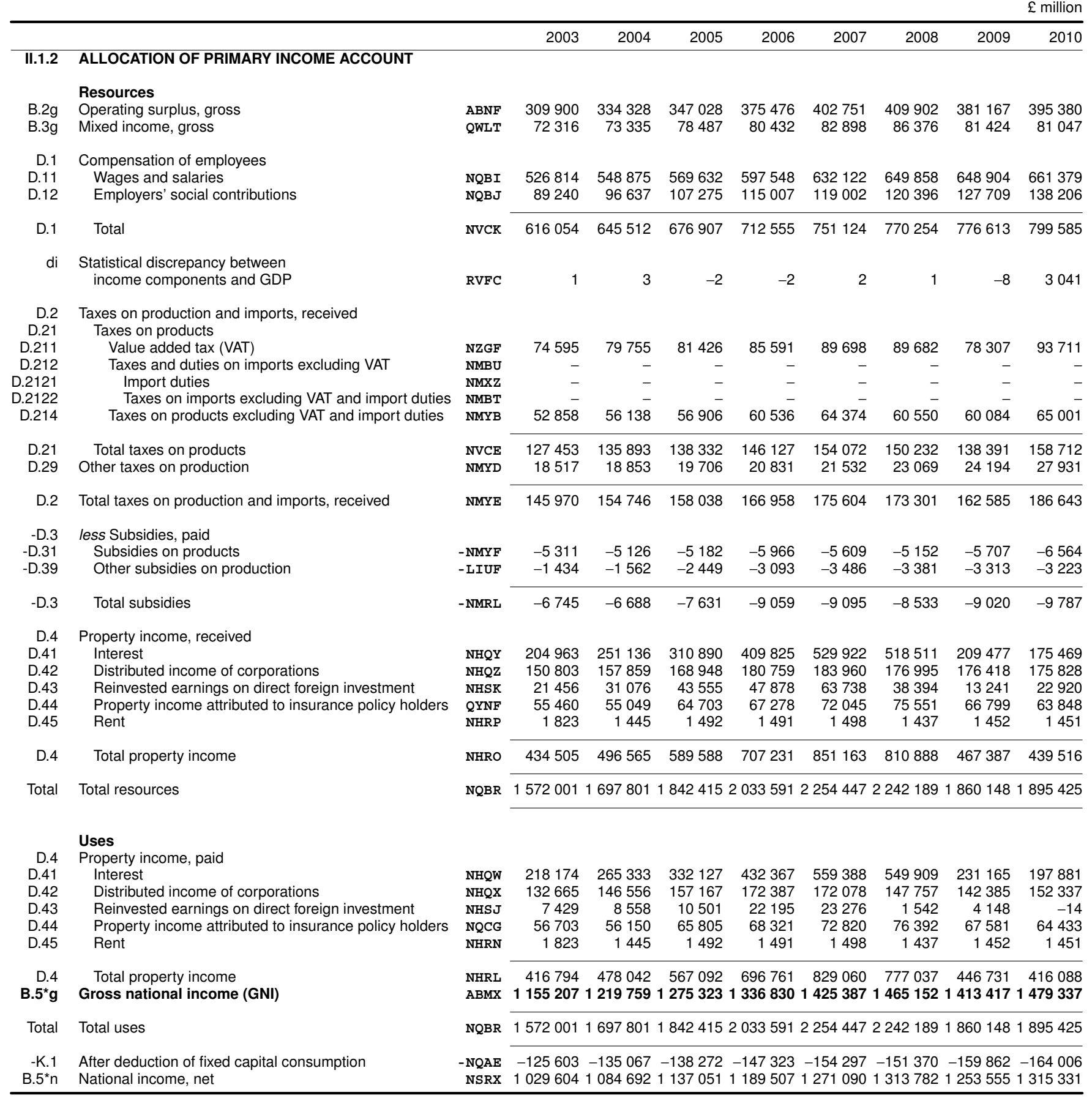




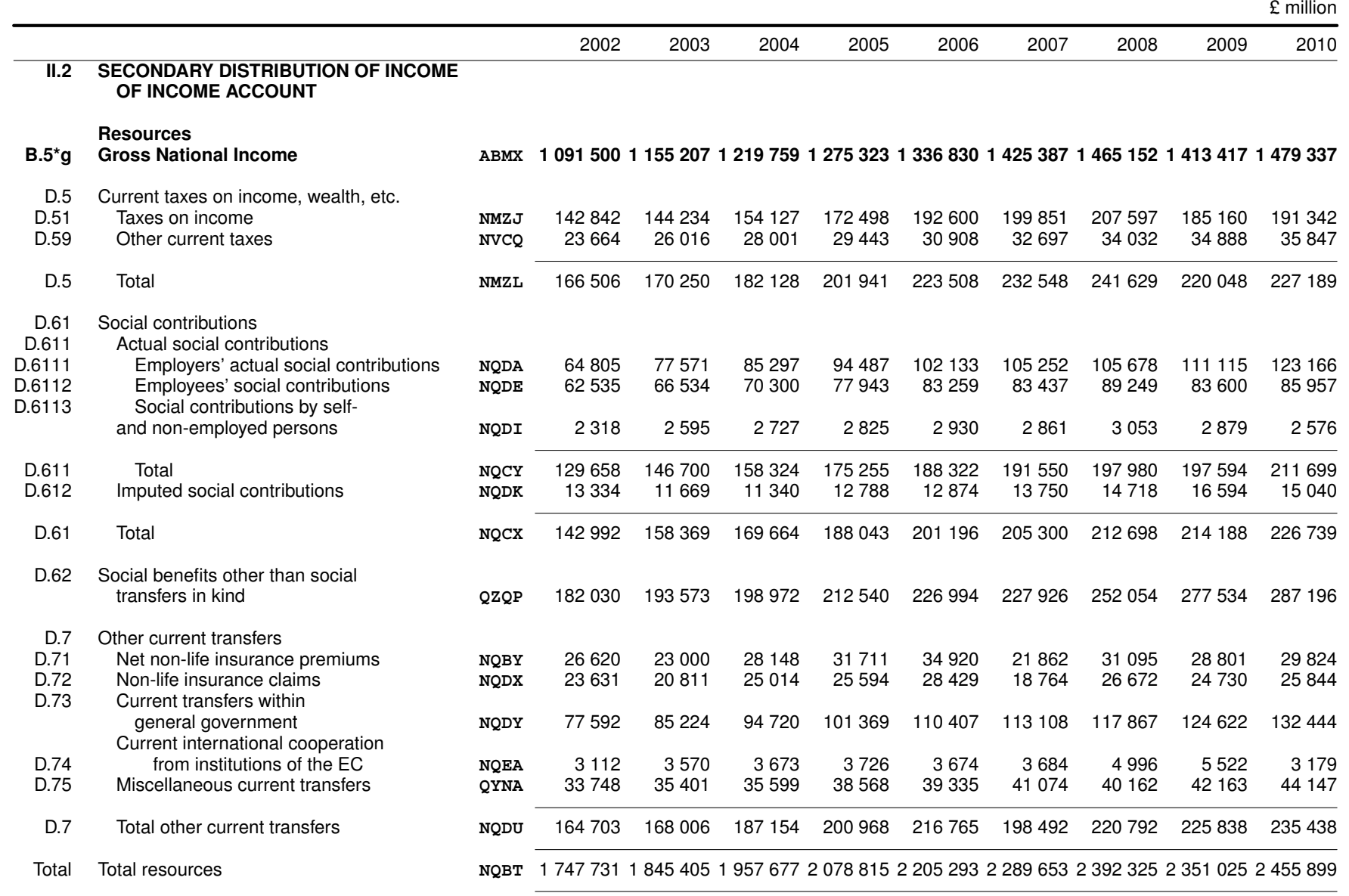

Uses

D.5 Current taxes on income, wealth etc.

D.51 Taxes on income

D.59 Other current taxes

D.5 Total

D.61 Social contributions

D.611 Actual social contributions

D.6111 Employers' actual social contributions

D.6112 Employees' actual social contributions

D.6113 Social contributions by self- and nonemployed persons

D.611 Total actual social contributions

D.612 Imputed social contributions

D.61 Total

D.62 Social benefits other than social transfers in kind

D.7 Other current transfers

D.71 Net non-life insurance premiums

D.72 Non-life insurance claims

D.73 Current transfers within general government Current international cooperation

D.74 to institutions of the EC

D.75 Miscellaneous current transfers Of which: GNP based fourth own resource

D.7 Total other current transfers

$\begin{array}{lllllllllll}\text { NQCR } & 142959 & 144303 & 154180 & 172541 & 192347 & 199797 & 207774 & 185053 & 191413\end{array}$

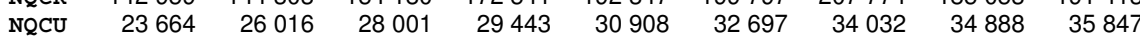

\begin{tabular}{llllllllll}
\cline { 2 - 4 } & 166623 & 170319 & 182181 & 201984 & 223255 & 232494 & 241806 & 219941 & 227260
\end{tabular}

\begin{tabular}{|c|c|c|c|c|c|c|c|c|c|}
\hline NQDB & 64805 & 77571 & 85297 & 94487 & 102133 & 105252 & 105678 & 111115 & 123166 \\
\hline NQDF & 62458 & 66490 & 70264 & 77929 & 83203 & 83411 & 89181 & 83428 & 85874 \\
\hline NQDJ & 2318 & 2595 & 2727 & 2825 & 2930 & 2861 & 3053 & 2879 & 2576 \\
\hline NQCZ & 129581 & 146656 & 158288 & 175241 & 188266 & 191524 & 197912 & 197422 & 211616 \\
\hline QZQQ & 13334 & 11669 & 11340 & 12788 & 12874 & 13750 & 14718 & 16594 & 15040 \\
\hline NQBS & 142915 & 158325 & 169628 & 188029 & 201140 & 205274 & 212630 & 214016 & 226656 \\
\hline NQDN & 183472 & 195050 & 200593 & 214237 & 228752 & 229787 & 254083 & 279785 & 289420 \\
\hline
\end{tabular}

B.6*g Gross national disposable income

Total Total uses

$\begin{array}{llllllllll}\text { NQDW } & 23631 & 20811 & 25014 & 25594 & 28429 & 18764 & 26672 & 24730 & 25844\end{array}$ $\begin{array}{llllllllll}\text { NQBZ } & 26620 & 23000 & 28148 & 31711 & 34920 & 21862 & 31095 & 28801 & 29824\end{array}$

$\begin{array}{llllllllll}\text { NNAF } & 77592 & 85224 & 94720 & 101369 & 110407 & 113108 & 117867 & 124622 & 132444\end{array}$

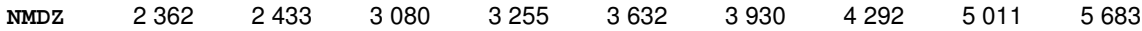

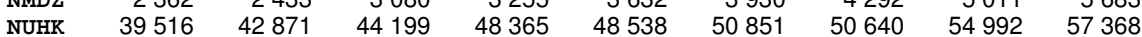
$\begin{array}{rrrrrrrrrr}\text { NUHK } & 39516 & 42871 & 44199 & 48365 & 48538 & 50851 & 50640 & 54992 & 57368 \\ \text { NMFH } & 5335 & 6772 & 7549 & 8732 & 8521 & 8323 & 8423 & 10555 & 10819\end{array}$ \begin{tabular}{llllllllll} 
NQDV & 169721 & 174339 & 195161 & 210294 & 225926 & 208515 & 230566 & 238156 & 251 \\
\hline
\end{tabular} NQCO 108500011473721210114126427113262201413583145324013991271461400 NQBT 174773118454051957677207881522052932289653239232523510252455899

-K.1 After deduction of fixed capital consumption -NQAE $\quad-121914 \quad-125603-135067-138272 \quad-147323-154297 \quad-151370-159862 \quad-164006$ B.6*n Disposable income, net NQCP 96308610217691075047112599911788971259286130187012392651297394 


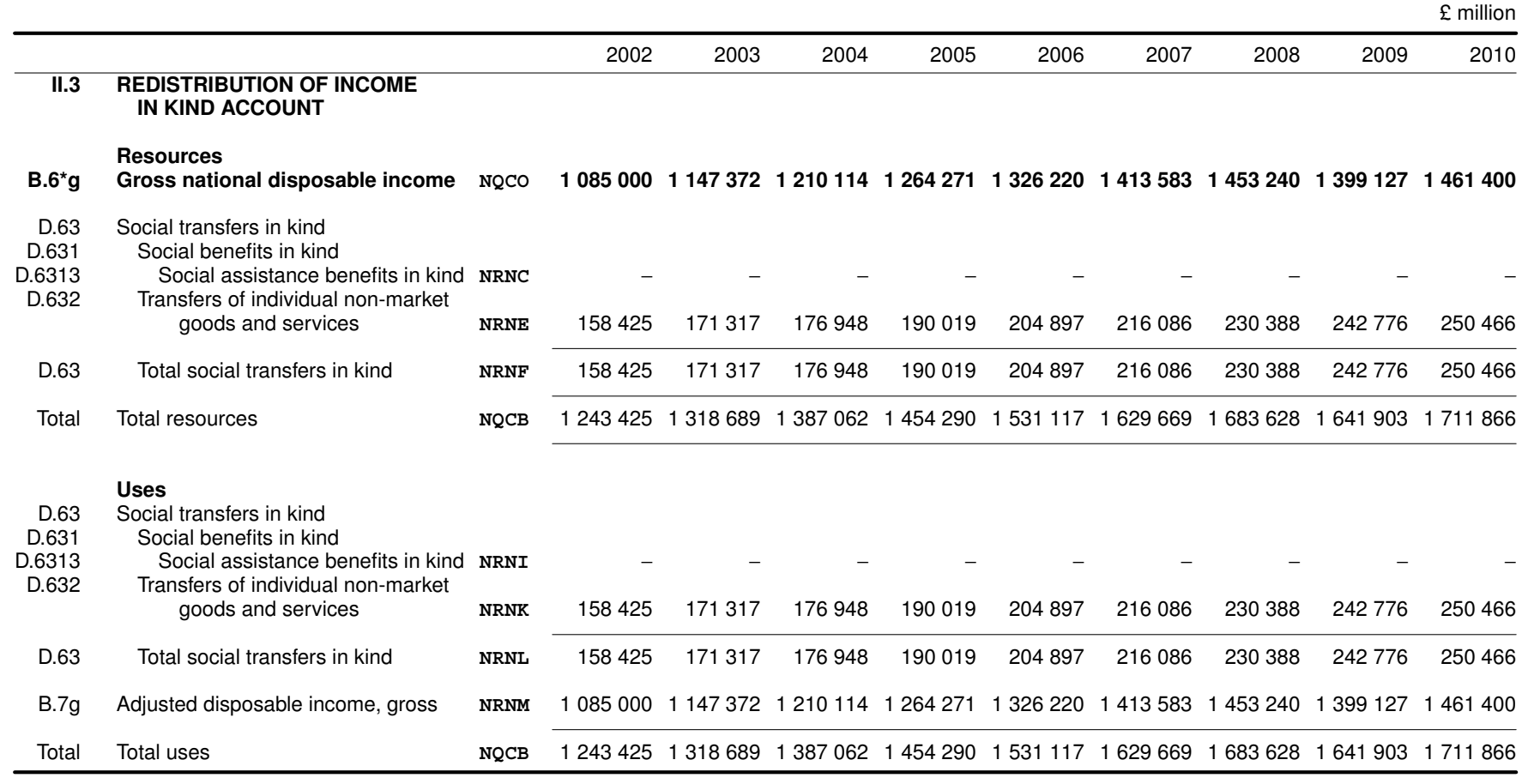




\begin{tabular}{lllllllllll}
\hline & £ million \\
\hline
\end{tabular}

II.4 USE OF INCOME ACCOUNT

II.4.1 USE OF DISPOSABLE INCOME ACCOUNT

\section{Resources}

B.6g Gross national disposable income

D.8 Adjustment for the change in net equity of households in pension funds

Total Total resources

Uses

P.3 Final consumption expenditure

P.31 Individual consumption expenditure

P.32 Collective consumption expenditure

P.3 Total

D.8 Adjustment for the change in net equity of households in pension funds

B.8g Gross saving

Total Total uses

-K.1 After deduction of fixed capital consumption

II.4.2 USE OF ADJUSTED DISPOSABLE INCOME ACCOUNT

\section{Resources}

B.7g Adjusted disposable income

D.8 Adjustment for the change in net equity of households in pension funds

Total Total resources

Uses

P.4 Actual final consumption

P.41 Actual individual consumption

P.42 Actual collective consumption

P.4 Total actual final consumption

D.8 Adjustment for the change in net equity of households in pension funds

B.8g Gross saving

Total Total uses
NQCO 108500011473721210114126427113262201413583145324013991271461400

$\begin{array}{llllllllll}\text { NVCI } & 17784 & 21377 & 26386 & 30881 & 29343 & 38871 & 27842 & 26547 & 35105\end{array}$

NVCW 110278411687491236500129515213555631452454148108214256741496505

NQEO $\quad 839074 \quad 885829 \quad 926555 \quad 97416810240611078328110841211010181150670$

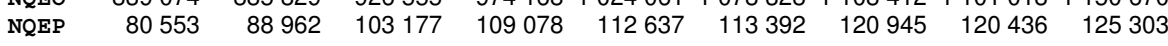

ABKW $919627 \quad 9747911029732108324611366981191720122935712214541275973$

$\begin{array}{llllllllll}\text { NQEL } & 17783 & 21373 & 26375 & 30826 & 29334 & 38834 & 27840 & 26506 & 35095\end{array}$

$\begin{array}{lllllllllll}\text { NQET } & 165374 & 172585 & 180393 & 181080 & 189531 & 221900 & 223885 & 177714 & 185437\end{array}$

NVCW 110278411687491236500129515213555631452454148108214256741496505

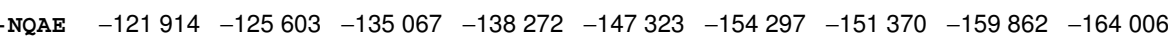

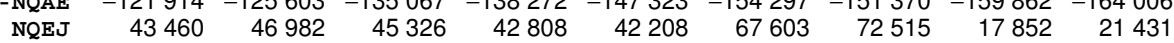

NRNM 108500011473721210114126427113262201413583145324013991271461400 $\begin{array}{llllllllll}\text { NVCI } & 17784 & 21377 & 26386 & 30881 & 29343 & 38871 & 27842 & 26547 & 35105\end{array}$ NVCW 110278411687491236500129515213555631452454148108214256741496505

\begin{tabular}{|c|c|c|c|c|c|c|c|c|c|}
\hline NQEO & 839074 & 885829 & 926555 & 974168 & 1024061 & 1078328 & 1108412 & 1101018 & 1150670 \\
\hline NRMZ & 80553 & 88962 & 103177 & 109078 & 112637 & 113392 & 120945 & 120436 & 125303 \\
\hline NRMX & 919627 & 974791 & 1029732 & 1083246 & 1136698 & 1191720 & 1229357 & 1221454 & 1275973 \\
\hline NQEL & 17783 & 21373 & 26375 & 30826 & 29334 & 38834 & 27840 & 26506 & 35095 \\
\hline NQET & 165374 & 172585 & 180393 & 181080 & 189531 & 221900 & 223885 & 177714 & 185437 \\
\hline VC & 102 & 749 & 500 & & & & 1 & 601 & \\
\hline
\end{tabular}




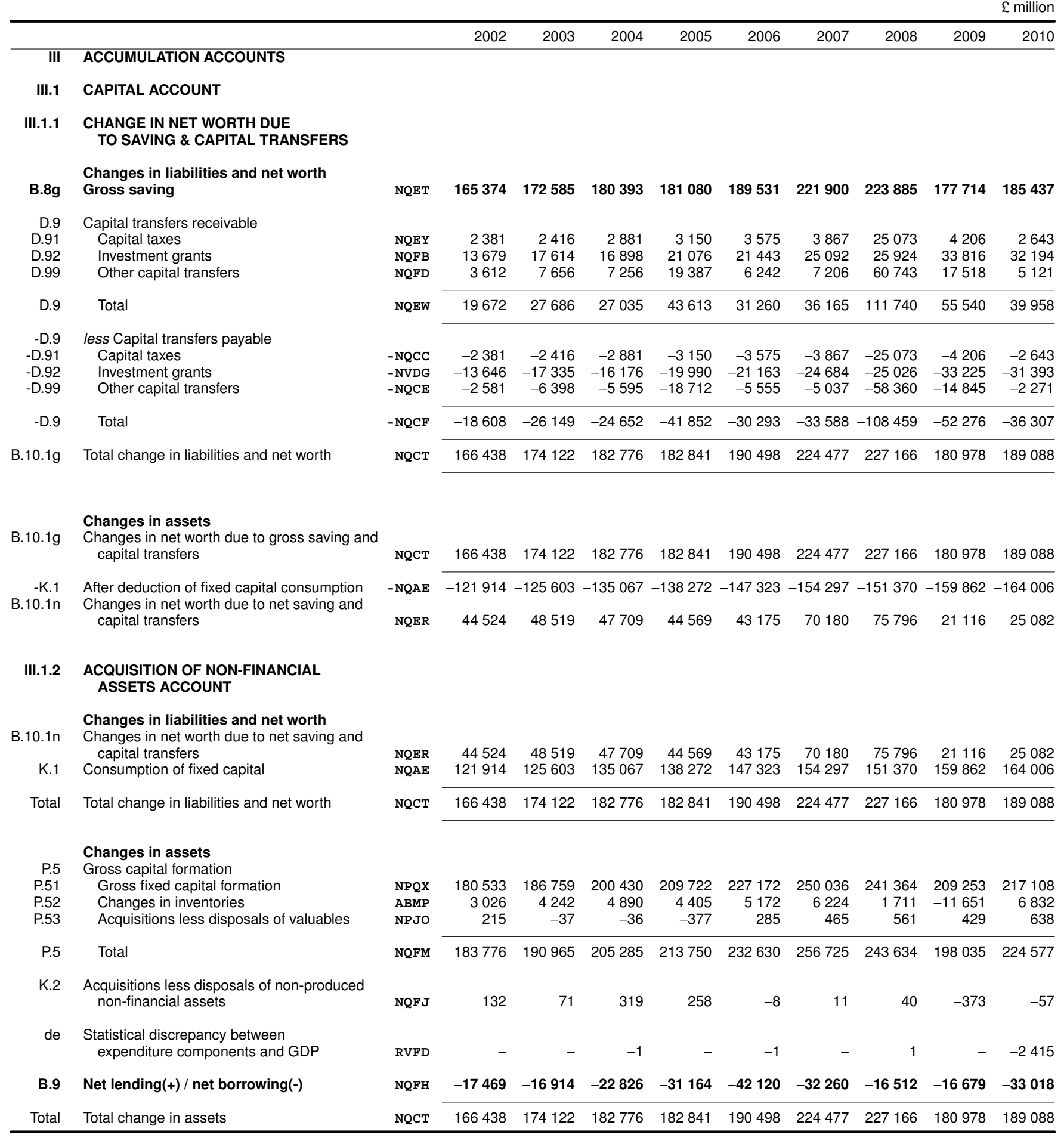




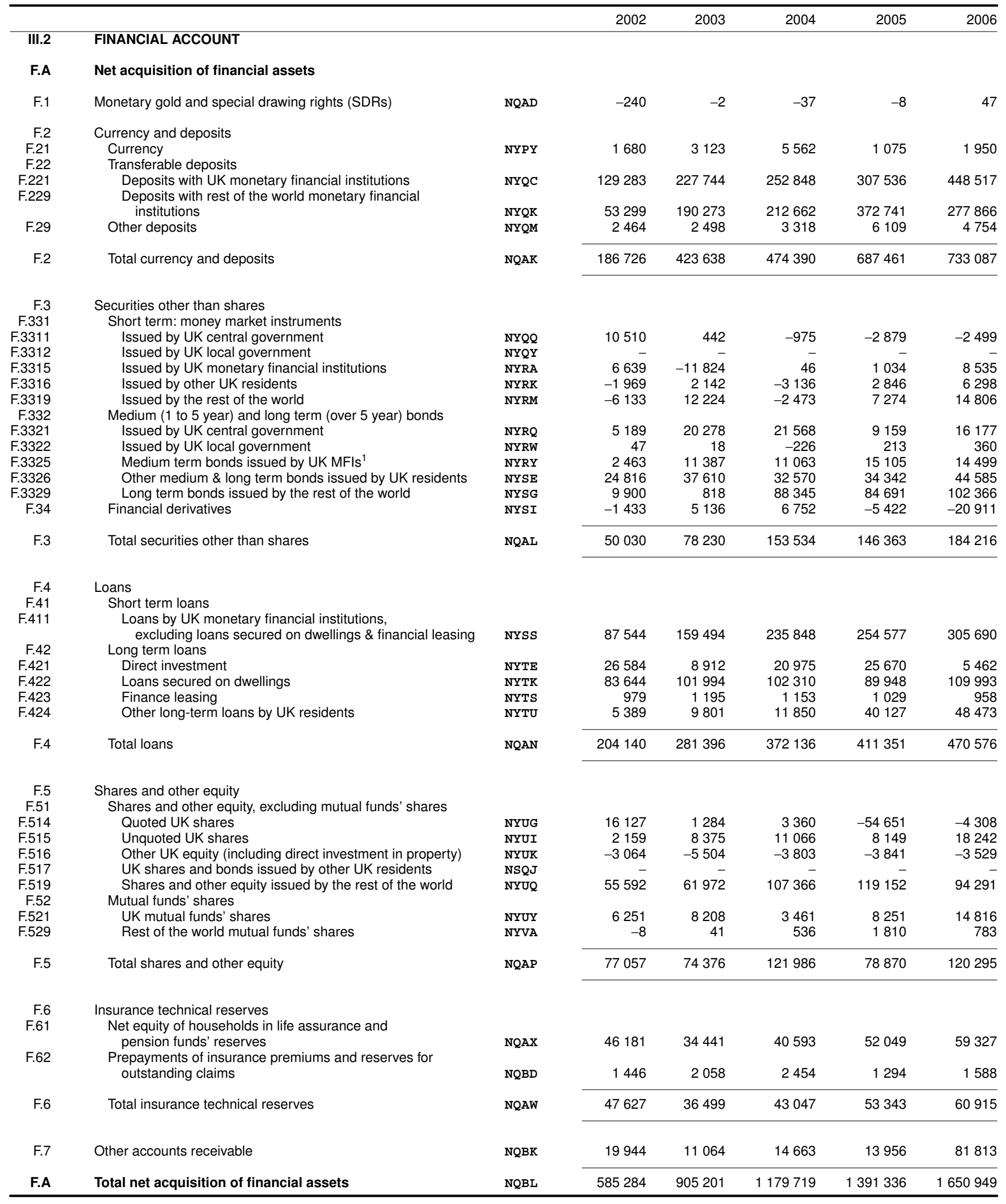

\footnotetext{
1 UK monetary financial institutions
} 


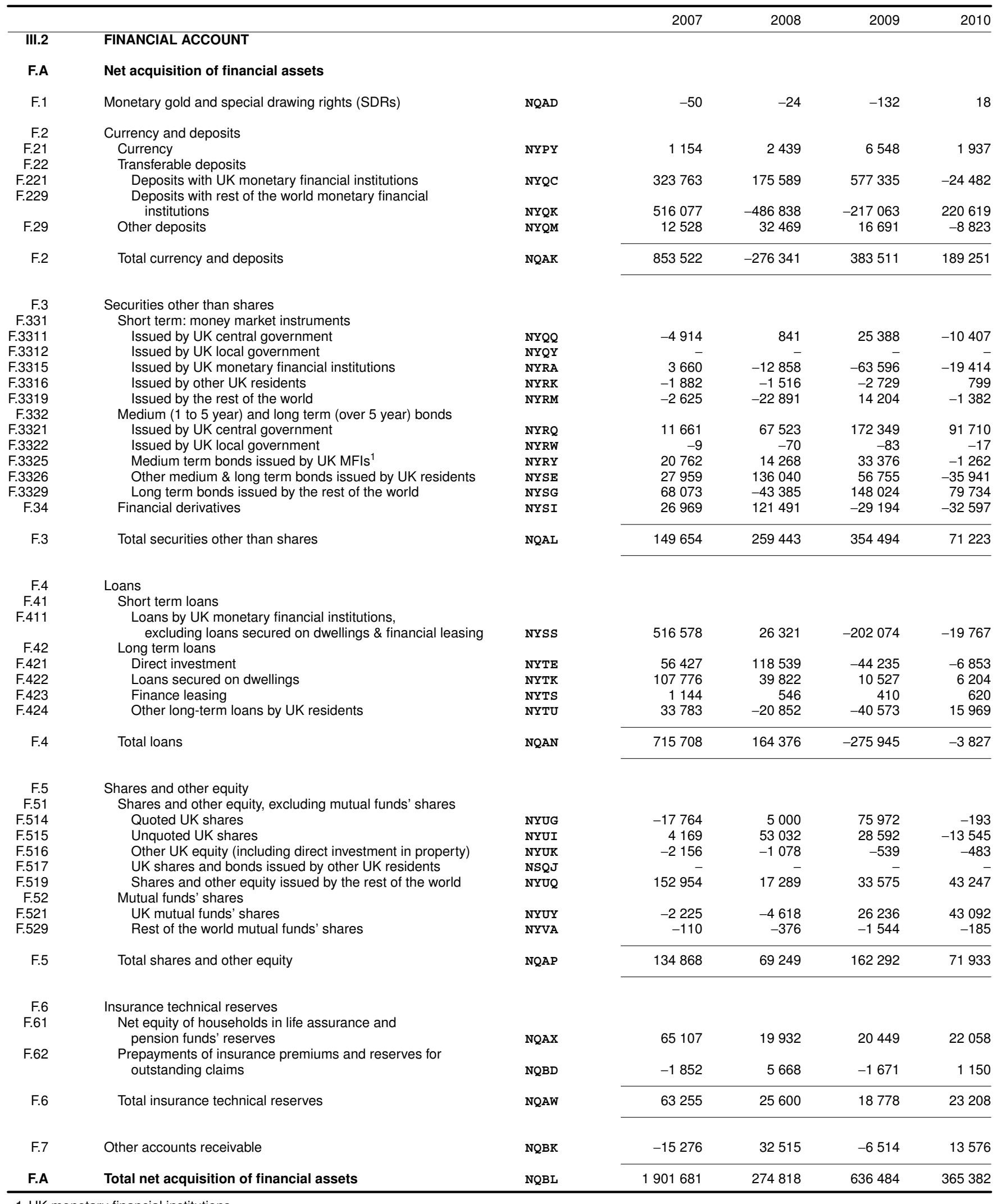

1 UK monetary financial institutions 
III.2 FINANCIAL ACCOUNT continued

F.L Net acquisition of financial liabilities

F.2 Currency and deposits

Currency

F.221 Deposits with UK monetary financial institutions

F.2 Total currency and deposits

F.3 Securities other than shares

F.331

F.3311

F.3312

F.3315

F.3316

F.332

F.3321

F.3322

F.3325

F.3326

F.34

F.3

F.4

F.41

F.411

F.419

F.42

F.421

F.422

F.423

F.424

F.429

F.4

Short term: money market instruments

Issued by UK central government

Issued by UK local government

ssued by UK monetary financial institution

Issued by other UK residents

Medium (1 to 5 year) and long term (over 5 year) bonds

Issued by UK central government

Medium term bonds issued by UK MFIs ${ }^{1}$

Other medium \& long term bonds issued by UK residents

Financial derivatives

Total securities other than shares

Loans

Short term loans

Loans by UK monetary financial institutions,

excl loans secured on dwellings \& financial leasing

Loans by rest of the world monetary financial institutions

Long term loans

Direct investment

Loans secured on dwellings

Finance leasing

Other long-term loans by UK residents

Other long-term loans by the rest of the world

Total loans

F.5

F.51

F.514

F.515

F.516

F.517

F.52

F.521

F.5

Shares and other equity

Shares and other equity, excluding mutual funds' shares Quoted UK shares

Unquoted UK shares

Other UK equity (including direct investment in property)

UK shares and bonds issued by other UK residents

Mutual funds' shares

UK mutual funds' shares

Total shares and other equity

F.6 Insurance technical reserves

F.61 Net equity of households in life assurance and

Net equity of households in
pension funds' reserves

Prepayments of insurance premiums and reserves for outstanding claims

F.6 Total insurance technical reserves

F.7 Other accounts payable

F.L Total net acquisition of financial liabilities

\section{B.9 Net lending / borrowing}

F.A Total net acquisition of financial assets

-F.L less Total net acquisition of financial liabilities

B.9f Net lending (+) / net borrowing (-), from financial account

dB.9f Statistical discrepancy between financial and non-financial accounts

B.9

Net lending (+) / net borrowing (-), from capital account
F.22 Transferable deposits

F.29 Other deposits

ssued by UK local government

$\begin{array}{lrrrrr}\text { NYPZ } & 1712 & 3174 & 5631 & 1125 & 1899 \\ \text { NYQD } & 218055 & 399447 & 541402 & 587184 & 782432 \\ \text { NYQN } & 2440 & 2730 & 2441 & 6052 & 5228 \\ \text { NQCK } & 222207 & 405351 & 549474 & 594361 & 789559\end{array}$

\begin{tabular}{lrrrrr} 
NYQR & 10330 & 2592 & 999 & -3902 & -1752 \\
NYQZ & - & - & - & - & - \\
NYRB & 25599 & -11489 & 8023 & -3490 & 53189 \\
NYRL & 8850 & -2181 & -3043 & 217 & 2800 \\
& & & & & \\
NYRR & 1555 & 31474 & 34176 & 39820 & 41100 \\
NYRX & 47 & 18 & -226 & 213 & 360 \\
NYRZ & 4238 & 25258 & 29810 & 37843 & 40534 \\
NYSF & 45132 & 101297 & 88869 & 113814 & 119195 \\
NYSJ & -274 & -75 & - & - & - \\
NQCM & 95477 & 146894 & 158608 & 184515 & 255426 \\
\cline { 2 - 7 } & & & & &
\end{tabular}

$\begin{array}{lrrrrr} & 70027 & 88503 & 123812 & 119769 & 185854 \\ \text { NYST } & -33874 & 70716 & 135773 & 221923 & 16120 \\ \text { NYTB } & & & & & \\ \text { NYTF } & 50445 & 12927 & 18354 & 44408 & 37337 \\ \text { NYTL } & 83644 & 101994 & 102310 & 89948 & 109993 \\ \text { NYTT } & 979 & 1195 & 1153 & 1029 & 958 \\ \text { NYTV } & 6847 & 10093 & 11919 & 41750 & 51644 \\ \text { NYTX } & -30 & 124 & 904 & 94 & 228 \\ & 178038 & 285552 & 394225 & 518921 & 402134\end{array}$

\begin{tabular}{lrrrrr} 
NYUH & 18881 & 14175 & 16625 & 3055 & 21070 \\
NYUU & 16008 & 27066 & 31381 & 49626 & 59371 \\
NYUL & -2316 & -5109 & -3180 & -3244 & -3062 \\
NSQK & - & - & - & - & - \\
NYUZ & 6259 & 8212 & 3489 & 8300 & 14866 \\
NQCS & 38832 & 44344 & 48315 & 57737 & 92245 \\
\cline { 2 - 6 }
\end{tabular}

NQCD

\begin{tabular}{lrrrrr} 
NQCD & 46180 & 34437 & 40582 & 51994 & 59318 \\
NQDD & 1781 & 687 & 3778 & 3969 & 6011 \\
\cline { 2 - 6 } NQCV & 47961 & 35124 & 44360 & 55963 & 65329 \\
\cline { 2 - 6 } NQDG & 18974 & 10497 & 14201 & 15074 & 80232 \\
\cline { 2 - 6 } NQDH & 601489 & 927762 & 1209183 & 1426571 & 1684925 \\
\cline { 2 - 6 }
\end{tabular}

1 UK monetary financial institutions 


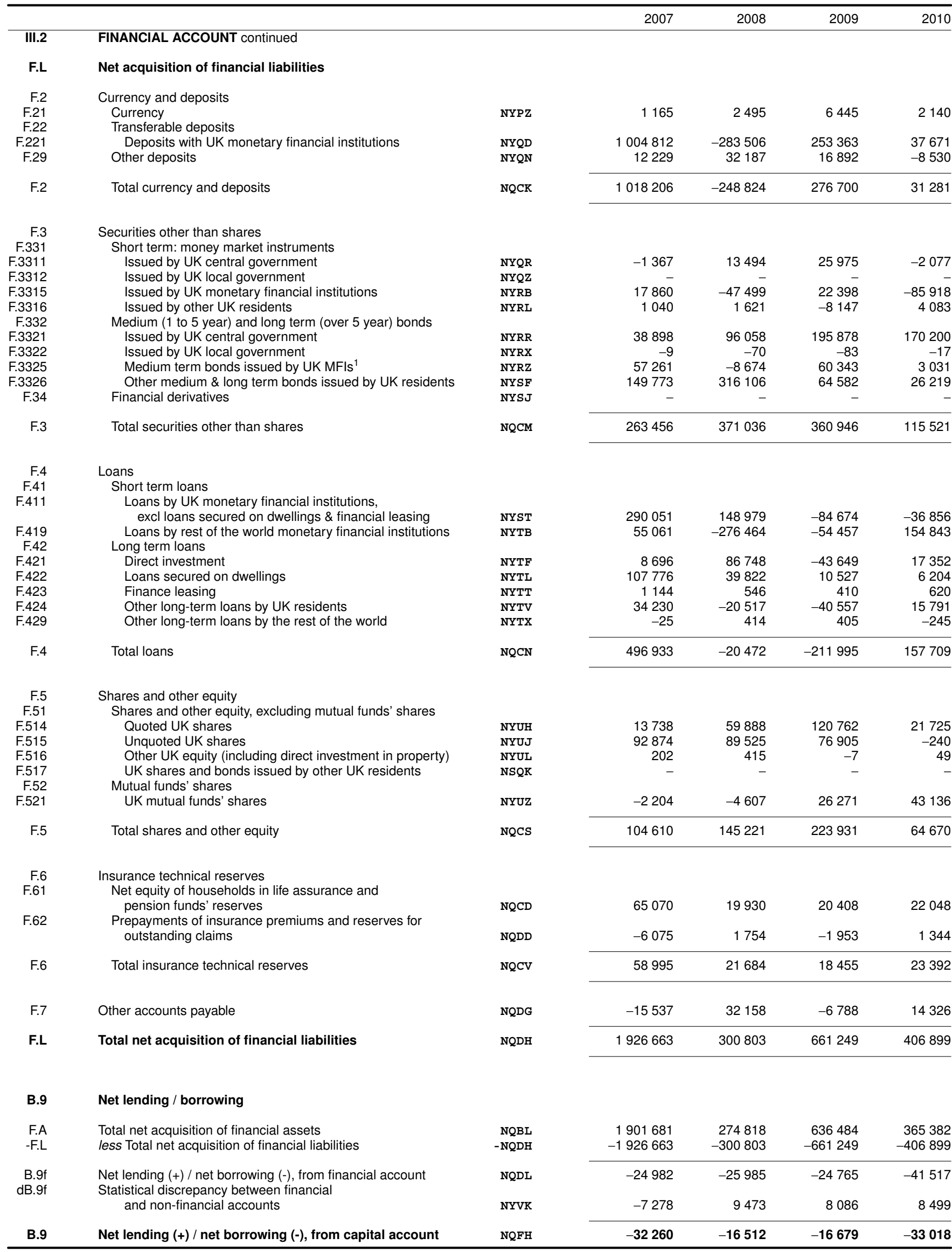

\footnotetext{
1 UK monetary financial institutions
} 


\begin{tabular}{|c|c|c|c|c|c|c|c|}
\hline & & & 2002 & 2003 & 2004 & 2005 & 2006 \\
\hline IV.3 & $\begin{array}{l}\text { FINANCIAL BALANCE SHEET } \\
\text { at end of period }\end{array}$ & & & & & & \\
\hline AN & Non-financial assets & CGJB & 5079.4 & 5523.5 & 6078.1 & 6299.0 & 6871.0 \\
\hline AF.A & Financial assets & & & & & & \\
\hline AF.1 & Monetary gold and special drawing rights (SDRs) & NYVN & 2.4 & 2.6 & 2.5 & 3.2 & 3.4 \\
\hline AF.2 & Currency and deposits & & & & & & \\
\hline $\begin{array}{l}\text { AF.21 } \\
\text { AF.22 }\end{array}$ & $\begin{array}{l}\text { Currency } \\
\text { Transferable deposits }\end{array}$ & NYVV & 40.5 & 43.6 & 49.1 & 50.1 & 52.0 \\
\hline $\begin{array}{l}\text { AF.221 } \\
\text { AF.229 }\end{array}$ & $\begin{array}{l}\text { Deposits with UK monetary financial institutions } \\
\text { institutions } \\
\text { Deposits with rest of the world monetary financial }\end{array}$ & NYVZ & 1595.3 & 1913.5 & 2125.9 & 2473.9 & 2957.8 \\
\hline AF.29 & $\begin{array}{l}\text { institutions } \\
\text { Other deposits }\end{array}$ & $\begin{array}{l}\text { NYWH } \\
\text { NYWJ }\end{array}$ & $\begin{array}{r}1203.3 \\
73.6\end{array}$ & $\begin{array}{r}1399.9 \\
75.1\end{array}$ & $\begin{array}{r}1605.3 \\
78.4\end{array}$ & $\begin{array}{r}2055.5 \\
85.5\end{array}$ & $\begin{array}{r}2189.5 \\
90.1\end{array}$ \\
\hline $\begin{array}{r}\text { AF.3 } \\
\text { AF.331 }\end{array}$ & $\begin{array}{l}\text { Securities other than shares } \\
\text { Short term: money market instruments }\end{array}$ & & & & & & \\
\hline AF.3311 & Issued by UK central government & NYWP & 21.2 & 22.1 & 21.2 & 18.3 & 15.8 \\
\hline AF.3312 & Issued by UK local government & NYWX & - & - & - & - & - \\
\hline AF.3315 & Issued by UK monetary financial institutions & NYWZ & 162.3 & 151.5 & 152.5 & 155.6 & 165.7 \\
\hline AF.3316 & Issued by other UK residents & NYXJ & 21.0 & 21.8 & 10.8 & 13.4 & 19.1 \\
\hline AF.3319 & Issued by the rest of the world & NYXL & 48.7 & 62.0 & 58.3 & 63.8 & 75.5 \\
\hline AF.332 & Medium ( 1 to 5 year) and long term (over 5 year) bonds & & & & & & \\
\hline AF.3321 & Issued by UK central government & NYXP & 254.8 & 265.8 & 289.1 & 313.5 & 315.8 \\
\hline AF.3322 & Issued by UK local government & NYXV & 0.8 & 0.8 & 0.6 & 0.8 & 1.2 \\
\hline AF.3325 & Medium term bonds issued by UK MFIs ${ }^{1}$ & NYXX & 37.4 & 53.3 & 63.8 & 80.0 & 91.0 \\
\hline AF.3326 & Other medium \& long term bonds issued by UK residents & NYYD & 288.7 & 319.8 & 355.0 & 417.4 & 472.0 \\
\hline AF.3329 & Long term bond issued by the rest of the world & NYYF & 538.2 & 550.1 & 611.3 & 717.2 & 798.3 \\
\hline AF.34 & Financial derivatives & NYYH & 0.2 & - & 1367.6 & 1650.4 & 1727.2 \\
\hline AF.3 & Total securities other than shares & NYWL & 1373.4 & 1447.4 & 2930.3 & 3430.4 & 3681.7 \\
\hline
\end{tabular}

AF.4 Loans

AF.41 Short term loans

AF.411 Loans by UK monetary financial institutions, excluding loans secured on dwellings \& financial leasing

Long term loans

NYYT

\begin{tabular}{rrrrr}
1142.5 & 1283.9 & 1487.7 & 1754.5 & 1969.0 \\
& & & & \\
176.2 & 175.3 & 205.5 & 222.6 & 224.1 \\
669.4 & 772.9 & 881.1 & 965.4 & 1077.2 \\
27.2 & 28.3 & 29.5 & 30.5 & 31.5 \\
164.8 & 177.0 & 196.6 & 207.0 & 247.8 \\
\hline 2180.0 & 2437.5 & 2800.3 & 3180.0 & 3549.6 \\
\hline
\end{tabular}

Direct investment

Loans secured on dwellings

Finance leasing

Other long-term loans by UK residents

AF.422

AF.423

AF.424

Total loans

Shares and other equity

Shares and other equity, excluding mutual funds' shares Quoted UK shares

Unquoted UK shares

Other UK equity (including direct investment in property)

UK shares and bonds issued by other UK residents

Shares and other equity issued by the rest of the world

Mutual funds' shares

UK mutual funds' shares

Rest of the world mutual fund share

AF.521

AF.529

Total shares and other equity

NYZF

NYZL

NYZT

NYZV

NYYP

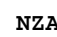

NZAL

NZAN

NSRC

NZAT

NZBB

NZBD

NYzZ

\begin{tabular}{rrrrr}
707.4 & 833.6 & 891.4 & 985.1 & 1065.3 \\
373.3 & 414.8 & 456.1 & 504.7 & 549.3 \\
97.2 & 105.8 & 114.6 & 130.8 & 122.1 \\
- & - & - & - & - \\
931.3 & 1049.0 & 1128.8 & 1348.3 & 1459.0 \\
& & & & \\
214.9 & 265.2 & 302.7 & 383.5 & 450.7 \\
1.4 & 1.4 & 1.7 & 4.1 & 6.0 \\
\hline 2325.5 & 2669.8 & 2895.4 & 3356.6 & 3652.4 \\
\hline
\end{tabular}

$\begin{array}{rr}\text { AF.6 } & \text { Insurance technical reserves } \\ \text { AF.61 } & \text { Net equity of households in life assurance and }\end{array}$

pension funds' reserves

Prepayments of insurance premiums and reserves for outstanding claims

AF.6 Total insurance technical reserves

NZBH

NZBN

NZBF

$\begin{array}{lllll}1384.1 & 1509.2 & 1603.2 & 1894.3 & 2071.7\end{array}$

$\begin{array}{lllll}50.1 & 53.2 & 55.7 & 57.0 & 58.6\end{array}$

\begin{tabular}{lllll}
1434.2 & 1562.4 & 1658.9 & 1951.3 & 2130.3 \\
\hline
\end{tabular}

AF.7 Other accounts receivable

AF.A Total financial assets

\begin{tabular}{rrrrr}
290.7 & 318.6 & 337.5 & 346.1 & 417.0 \\
\hline 10518.9 & 11870.3 & 14483.6 & 16932.6 & 18723.8
\end{tabular}

1 UK monetary financial institutions 


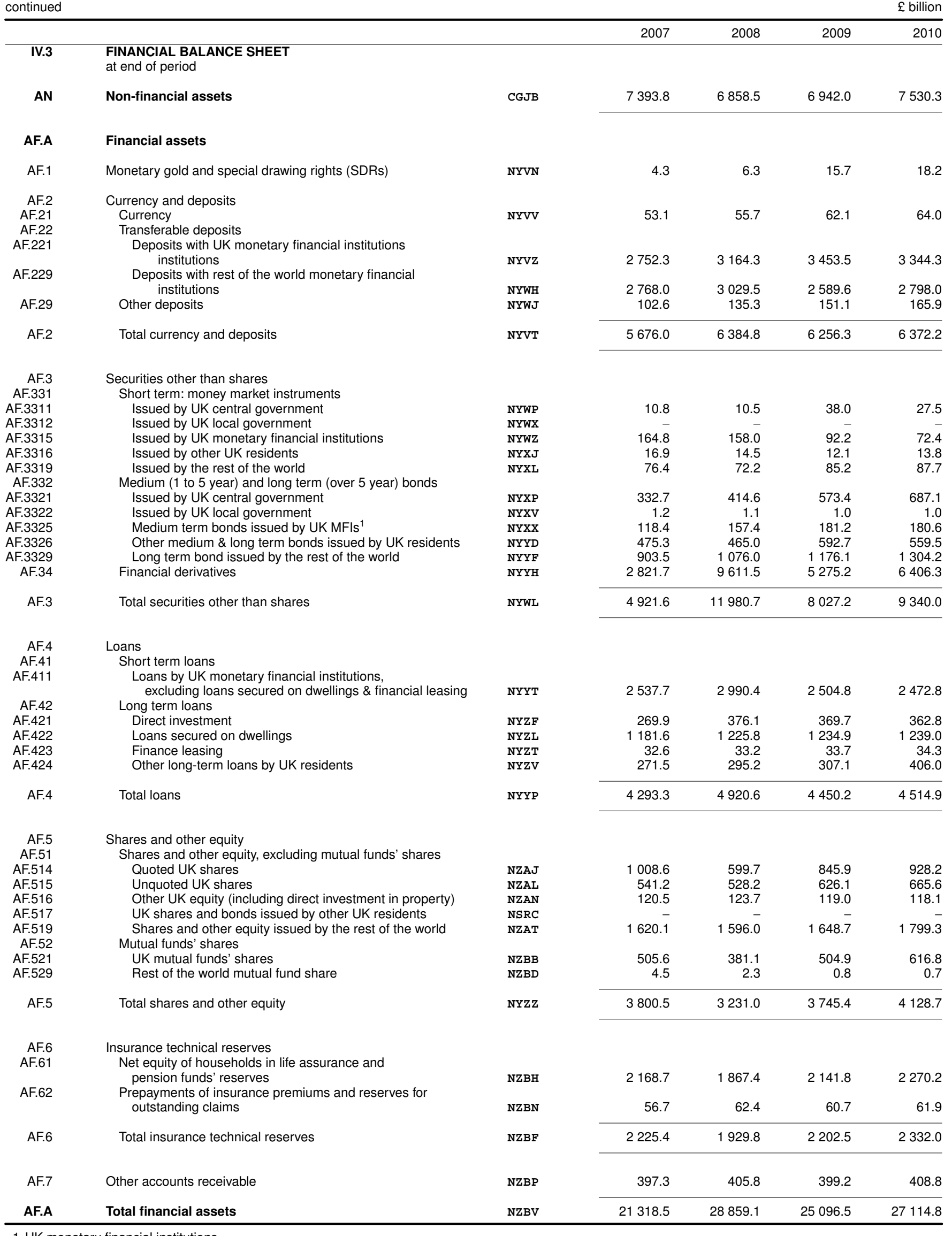

1 UK monetary financial institutions 


\begin{tabular}{|c|c|c|c|c|c|c|c|}
\hline & & & 2002 & 2003 & 2004 & 2005 & 2006 \\
\hline IV.3 & $\begin{array}{l}\text { FINANCIAL BALANCE SHEET continued } \\
\text { at end of period }\end{array}$ & & & & & & \\
\hline AF.L & Financial liabilities & & & & & & \\
\hline AF.2 & Currency and deposits & & & & & & \\
\hline AF.21 & Currency & NYVW & 41.1 & 44.2 & 49.8 & 50.9 & 52.8 \\
\hline AF.22 & Transferable deposits & & & & & & \\
\hline AF.221 & Deposits with UK monetary financial institutions & NYWA & 3034.7 & 3518.7 & 3981.1 & 4673.7 & 5328.5 \\
\hline AF.2 & Total currency and deposits & NYVU & 3151.1 & 3639.9 & 4110.4 & 4811.0 & 5472.7 \\
\hline $\begin{array}{r}\text { AF.3 } \\
\text { AF.331 }\end{array}$ & Securities other than shares & & & & & & \\
\hline $\begin{array}{r}\text { AF.331 } \\
\text { AF } 3311\end{array}$ & Short term: money market instruments & & & & & & \\
\hline AF.3311 & Issued by UK central government & NYWQ & 21.4 & 24.0 & 25.0 & 21.1 & 19.4 \\
\hline AF.3312 & Issued by UK local government & NYWY & - & - & - & - & - \\
\hline AF.3315 & Issued by UK monetary financial institutions & NYXA & 302.6 & 282.1 & 283.2 & 291.7 & 327.7 \\
\hline AF.3321 & Issued by UK central government & NYXQ & 311.1 & 331.9 & 372.9 & 424.2 & 451.3 \\
\hline AF.3322 & Issued by UK local government & NYXW & 0.8 & 0.8 & 0.6 & 0.8 & 1.2 \\
\hline AF.3325 & Medium term bonds issued by UK MFIs ${ }^{1}$ & NYXY & 81.0 & 107.0 & 134.7 & 175.4 & 205.8 \\
\hline AF.3326 & Other medium \& long term bonds issued by UK residents & NYYE & 517.6 & 615.5 & 716.8 & 880.9 & 1001.1 \\
\hline AF.34 & Financial derivatives & NYYI & - & - & 1373.2 & 1661.0 & 1764.0 \\
\hline AF.3 & Total securities other than shares & NYWM & 1286.2 & 1407.0 & 2939.8 & 3490.8 & 3805.9 \\
\hline AF.4 & Loans & & & & & & \\
\hline AF.41 & Short term loans & & & & & & \\
\hline AF.411 & $\begin{array}{l}\text { Loans by UK monetary financial institutions, excluding } \\
\text { loans secured on dwellings \& financial leasing }\end{array}$ & NYYU & 814.1 & 885.5 & 991.3 & 1112.5 & 1259.9 \\
\hline AF.419 & Loans by rest of the world monetary financial institutions & NYZC & 438.9 & 512.2 & 637.6 & 887.3 & 872.3 \\
\hline AF.42 & Long term loans & & & & & & \\
\hline AF.421 & Direct investment & NYZG & 284.1 & 280.6 & 305.1 & 358.7 & 376.8 \\
\hline AF.422 & Loans secured on dwellings & NYZM & 669.4 & 772.9 & 881.1 & 965.4 & 1077.2 \\
\hline AF.423 & Finance leasing & NYZU & 27.2 & 28.3 & 29.5 & 30.5 & 31.5 \\
\hline AF.424 & Other long-term loans by UK residents & NYZW & 157.5 & 169.9 & 189.2 & 199.6 & 242.4 \\
\hline AF.5 & Shares and other equity & & & & & & \\
\hline AF.51 & Shares and other equity, excluding mutual funds' shares & & & & & & \\
\hline AF.514 & Quoted UK shares & NZAK & 1126.1 & 1334.0 & 1441.7 & 1644.5 & 1804.5 \\
\hline AF.515 & Unquoted UK shares & NZAM & 609.8 & 670.2 & 729.5 & 853.4 & 1002.2 \\
\hline AF.516 & Other UK equity (including direct investment in property) & NZAO & 113.1 & 121.7 & 132.4 & 149.2 & 142.3 \\
\hline AF.517 & UK shares and bonds issued by other UK residents & NSRD & - & - & - & - & - \\
\hline AF.52 & Mutual funds' shares & & & & & & \\
\hline AF.521 & UK mutual funds' shares & NZBC & 215.8 & 266.3 & 303.9 & 385.0 & 452.4 \\
\hline AF.5 & Total shares and other equity & NZAA & 2064.7 & 2392.2 & 2607.5 & 3032.1 & 3401.4 \\
\hline AF.6 & Insurance technical reserves & & & & & & \\
\hline AF.61 & $\begin{array}{l}\text { Net equity of households in life assurance and } \\
\text { pension funds' reserves }\end{array}$ & NZBI & 1384.3 & 1509.4 & 1603.4 & 1894.5 & 2071.9 \\
\hline AF.62 & Prepayments of insurance premiums and reserves for & & & & & & \\
\hline & outstanding claims & NZBO & 62.8 & 63.5 & 67.2 & 71.2 & 77.2 \\
\hline AF.6 & Total insurance technical reserves & NZBG & 1447.1 & 1572.9 & 1670.6 & 1965.7 & 2149.1 \\
\hline AF.7 & Other accounts payable & NZBQ & 288.6 & 315.8 & 335.6 & 345.3 & 415.1 \\
\hline AF.L & Total financial liabilities & NZBW & 10630.9 & 11979.4 & 14700.9 & 17202.2 & 19107.6 \\
\hline
\end{tabular}

BF.90 Net financial assets / liabilities

AF.A Total financial assets

-AF.L less Total financial liabilities

NZBV

\begin{tabular}{rrrrr}
10518.9 & 11870.3 & 14483.6 & 16932.6 & 18723.8 \\
-10630.9 & -11979.4 & -14700.9 & -17202.2 & -19107.6 \\
\hline-112.0 & -109.2 & -217.3 & -269.6 & -383.8
\end{tabular}

Net worth

$\begin{aligned} \text { AN } & \text { Non-financial assets } \\ \text { BF.90 } & \text { Net financial assets (+) / liabilities (-) } \\ \text { B.90 } & \text { Net worth }\end{aligned}$

\begin{tabular}{|c|c|c|c|c|c|}
\hline CGJB & 5079.4 & 5523.5 & 6078.1 & 6299.0 & $\begin{array}{r}6871.0 \\
-383.8\end{array}$ \\
\hline NQFT & -112.0 & -109.2 & -217.3 & -269.6 & \\
\hline$c$ & 4967.4 & 5414.3 & 5860.8 & 6029.4 & 6487 \\
\hline
\end{tabular}

1 UK monetary financial institutions 


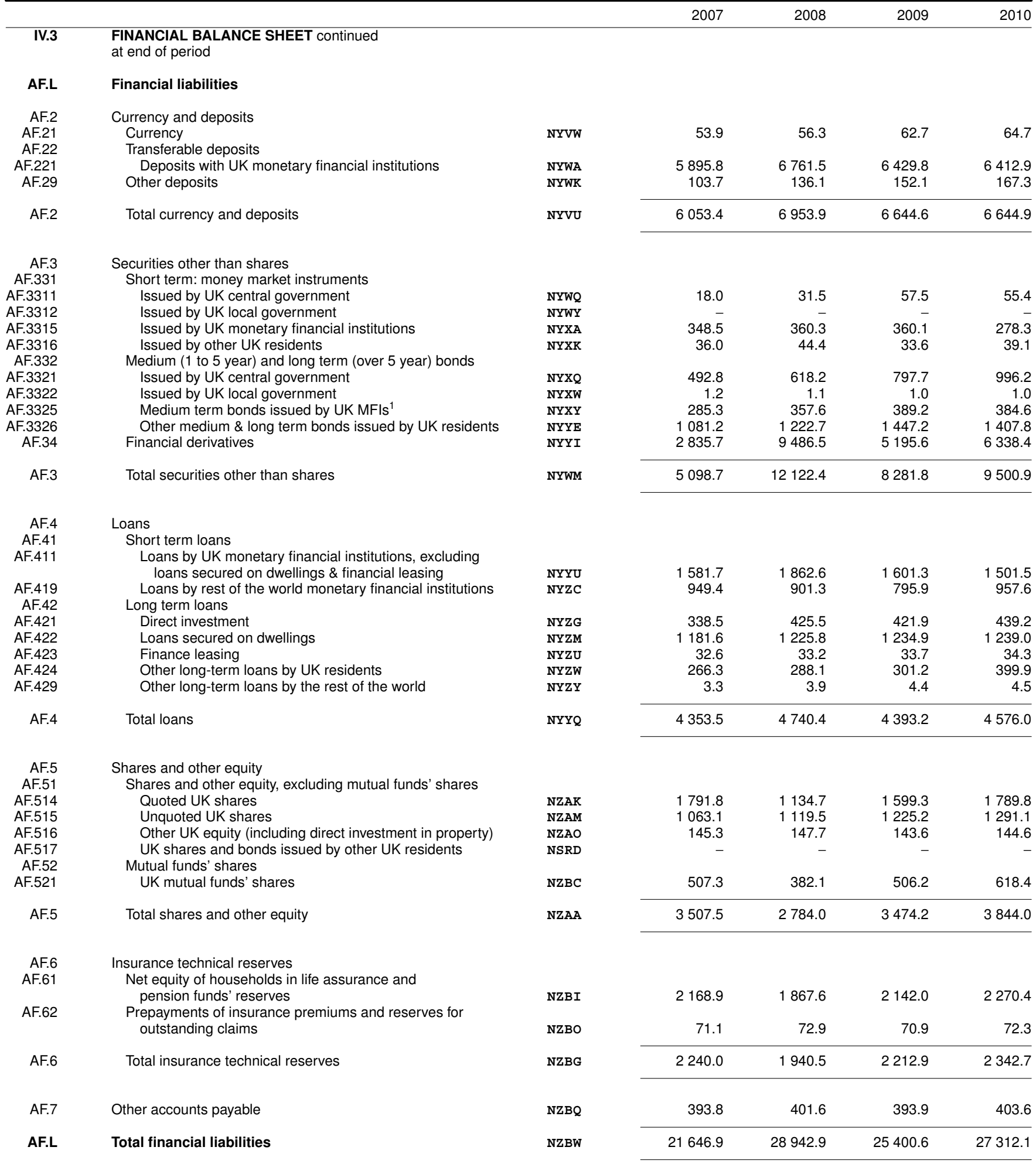

BF.90 Net financial assets / liabilities

AF.A Total financial assets

-AF.L less Total financial liabilities

\begin{tabular}{rrrr}
21318.5 & 28859.1 & 25096.5 & 27114.8 \\
-21646.9 & -28942.9 & -25400.6 & -27312.1 \\
\hline-328.4 & -83.8 & -304.1 & -197.3
\end{tabular}

Net worth

$\begin{aligned} \text { AN } & \text { Non-financial assets } \\ \text { BF.90 } & \text { Net financial assets (+) / liabilities (-) } \\ \text { B.90 } & \text { Net worth }\end{aligned}$

\begin{tabular}{rrrr}
7393.8 & 6858.5 & 6942.0 & 7530.3 \\
-328.4 & -83.8 & -304.1 & -197.3 \\
\hline 7065.4 & 6774.7 & 6637.9 & 7333.0 \\
\hline
\end{tabular}

1 UK monetary financial institutions 
total Non-financial Financial General Households Not the world services economy corporations corporations government \& NPISH sector

$\begin{array}{lllllrl}\text { S.1 } & \text { S.11 } & \text { S.12 } & \text { S.13 } & \text { S.14+S.15 } & \text { S.N } & \text { S.2 }\end{array}$

\section{Current accounts}

I PRODUCTION / EXTERNAL

0 ACCOUNT OF GOODS AND SERVICES

P.7 Imports of goods and services

P.6 Exports of goods and services

P.1 Output at basic prices

P.2 Intermediate consumption

D.21-D.31 Taxes less subsidies on products

II.1.1 GENERATION OF INCOME

B.1g Gross domestic product, value added at market prices

B.11 External balance of goods and services

II.1.2 ALLOCATION OF PRIMARY INCOME

D.1 Compensation of employees

D.21-D.31 Taxes less subsidies on products

D.29-D.39 Other taxes less subsidies on production

B.2g Operating surplus, gross

B.3g Mixed income, gross

Statistical discrepancy between

di income components and GDP

D.4 Property income

II.2 SECONDARY DISTRIBUTION OF INCOME

National income,

B.5g balance of primary incomes, gross

D.5 Current taxes on income, wealth etc

D.61 Social contributions

Social benefits

D.62 other than social transfers in kind

D.7 Other current transfers

II.3 REDISTRIBUTION OF INCOME IN KIND

B.6g Disposable income, gross

D.63 Social transfers in kind

II.4 USE OF INCOME

B.7g Adjusted disposable income, gross

B.6g Disposable income, gross

P.4 Actual final consumption

P.3 Final consumption expenditure

Adjustment for change in households

D.8 net equity in pension funds

\section{Accumulation accounts}

III.1.1 CHANGE IN NET WORTH DUE TO SAVING AND CAPITAL TRANSFERS

B.8g Saving, gross

B.12 Current external balance

D.9 Capital transfers receivable

D.9 Capital transfers payable

III.1.2 ACQUISITION ON NON-FINANCIAL ASSETS

Changes in liabilities and net worth Changes in net worth due to saving and

B.10.1.g capital transfers

P.51 Gross fixed capital formation

-K.1 (Consumption of fixed capital)

P.52 Changes in inventories

P.53 Acquisitions less disposals of valuables Acquisitions less disposals of

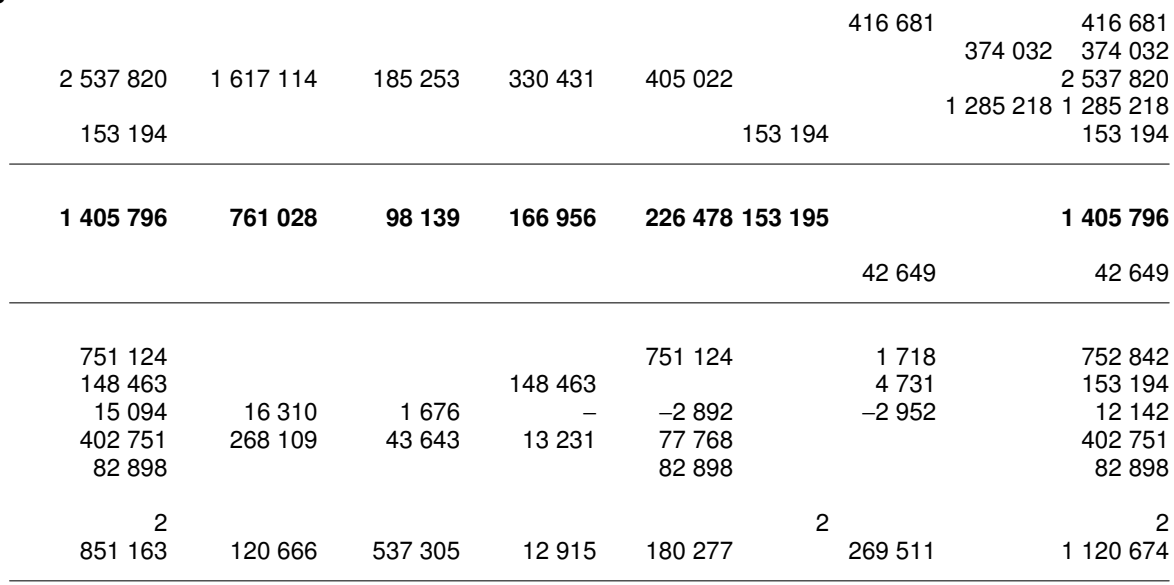

$\begin{array}{rrrrrrr}1425387 & 208266 & 42015 & 157863 & 1017241 & 2 & \\ 232548 & & & 232548 & & 549 & 1425387 \\ 205300 & 4824 & 84376 & 115582 & 518 & - & 233097 \\ 227926 & & & & 227926 & 1861 & 229787 \\ 198492 & 4007 & 22277 & 117610 & 54598 & 20319 & 218811\end{array}$

.2 non-produced non-financial assets Statistical discrepancy between

de expenditure components and GDP

III.2 FINANCIAL ACCOUNT

B.9 Net lending(+) / net borrowing(-) Changes in liabilities

F.2 Currency and deposits

F.3 Securities other than shares

F.4 Loans

F.5 Shares and other equity

F.6 Insurance technical reserves

F.7 Other accounts payable

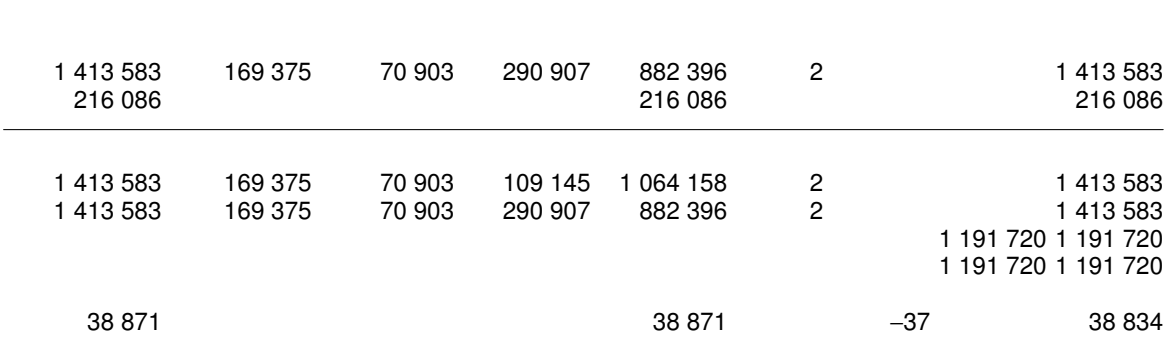

$\begin{array}{rrrrrrr}221900 & 169375 & 32069 & -4247 & 24701 & 2 & \\ 36165 & 8420 & 388 & 16918 & 10439 & 34826 & 1233 \\ -33588 & -880 & -388 & -27326 & -4994 & -3810 & 34826 \\ & & & & & & -37398\end{array}$

$\begin{array}{rrrrrrr}224477 \quad 32069 & -14655 & 30146 & 2 & 32249 & & 256726 \\ & & & & & 25036 & 250036 \\ & & & 6224 & 6224 \\ & & & 465 & 465\end{array}$

$\begin{array}{rrrrrrrr}-32260 & 38279 & 23611 & -38331 & -55821 & 2 & 32260 & - \\ 1018206 & & & 1010154 & 8052 & - & 516111 & 1534317 \\ 263456 & 24652 & 199702 & 37522 & 1580 & 92417 & 355873 \\ 496933 & 128830 & 244956 & 2015 & 121132 & 282507 & 779440 \\ 104610 & 60222 & 44388 & & - & 152844 & 257454 \\ 58995 & & 58995 & & -2059 & -6472 & 506 & -15031 \\ -15537 & 2947 & -9953 & -2059 & \end{array}$


UK

total Non-financial Financial General Households Not the world services economy corporations corporations government \& NPISH sector

S. 1 S.11 S.12 S.13 S.14+S.15 S.N S.2

\section{Current accounts}

I PRODUCTION / EXTERNAL

0 ACCOUNT OF GOODS AND SERVICES

P.7 Imports of goods and services

P.6 Exports of goods and services

P.1 Output at basic prices

P2 Intermediate consumption

D.21-D.31 Taxes less subsidies on products

B.1g Gross domestic product, value added at market prices

B.11 External balance of goods and services

II.1.1 GENERATION OF INCOME

D.1 Compensation of employees

D.21-D.31 Taxes less subsidies on products

D.29-D.39 Other taxes less subsidies on production

B.2g Operating surplus, gross

B.3g Mixed income, gross

Statistical discrepancy between

di income components and GDP

II.1.2 ALLOCATION OF PRIMARY INCOME

D.4 Property income

National income

B. $5 \mathrm{~g}$ balance of primary incomes, gross

II.2 SECONDARY DISTRIBUTION OF INCOME

D.5 Current taxes on income, wealth etc

D.61 Social contributions

Social benefits

D. other than social transfers in kind

D.7 Other current transfers

B.6g Disposable income, gross

II.3 REDISTRIBUTION OF INCOME IN KIND

B.7g Adjusted disposable income, gross

D.63 Social transfers in kind

II.4 USE OF INCOME

B.6g Disposable income, gross

P.4 Actual final consumption

P.3 Final consumption expenditure Adjustment for change in households

D.8 net equity in pension funds

B.8g Saving, gross

B.12 Current external balance

Accumulation accounts

CHANGE IN NET WORTH

III.1.1 DUE TO SAVING AND CAPITAL TRANSFERS

D.9 Capital transfers receivable

D.9 Capital transfers payable

Changes in net worth due to saving

B.10.1.g and capital transfers

III.1.2 ACQUISITION OF NON-FINANCIAL ASSETS

Changes in assets

P.51 Gross fixed capital formation

-K.1 (Consumption of fixed capital)

P.52 Changes in inventories

P.53 Acquisitions less disposals of valuables Acquisitions less disposals of

K.2 non-produced non-financial assets Statistical discrepancy between

de expenditure components and GDP

B.9 Net lending(+)/ net borrowing(-)

III.2 FINANCIAL ACCOUNT: changes in assets

F.1 Monetary gold and SDRs

F.2 Currency and deposits

F.3 Securities other than shares

F.4 Loans

F.5 Shares and other equity

F.6 Insurance technical reserves

F.7 Other accounts receivable

Statistical discrepancy between non-fin

dB.9f and financial transactions

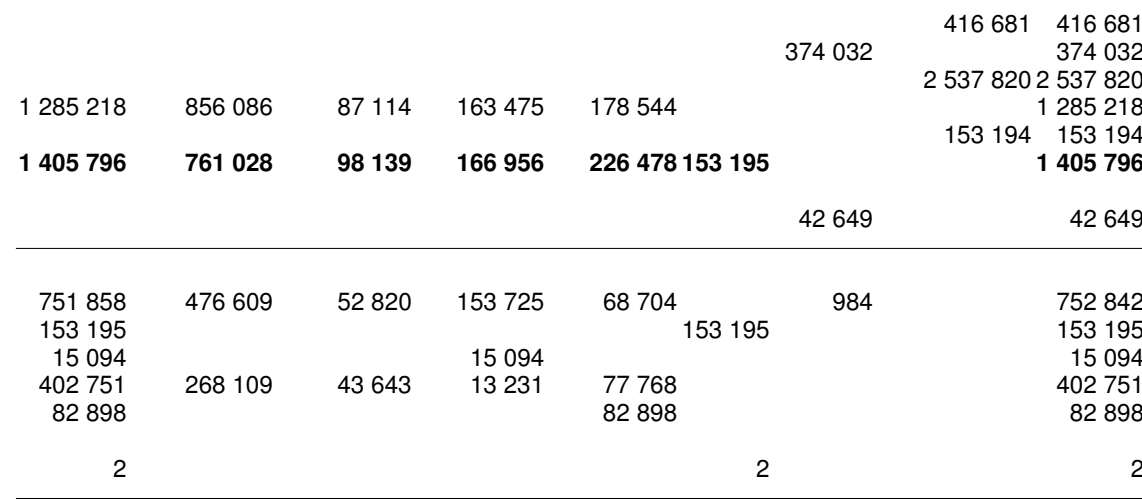

\begin{tabular}{rrrrrrr}
\hline 829060 & 180509 & 538933 & 34792 & 74826 & 291614 & 1120674 \\
1425387 & 208266 & 42015 & 157863 & 1017241 & 2 & 1425387
\end{tabular}

\begin{tabular}{|c|c|c|c|c|c|c|}
\hline $\begin{array}{l}232494 \\
205274\end{array}$ & 38403 & 9866 & 1111 & $\begin{array}{l}183114 \\
205274\end{array}$ & $\begin{array}{r}603 \\
26\end{array}$ & $\begin{array}{l}233097 \\
205300\end{array}$ \\
\hline $\begin{array}{r}229787 \\
208515 \\
1413583\end{array}$ & $\begin{array}{r}4824 \\
4495 \\
169375\end{array}$ & $\begin{array}{l}45542 \\
22357 \\
70903\end{array}$ & $\begin{array}{l}178407 \\
153178 \\
290907\end{array}$ & $\begin{array}{r}1014 \\
28485 \\
882396\end{array}$ & 10296 & $\begin{array}{r}229787 \\
218811 \\
1413583\end{array}$ \\
\hline $\begin{array}{r}1413583 \\
216086\end{array}$ & 169375 & 70903 & $\begin{array}{l}109145 \\
181762\end{array}$ & $\begin{array}{r}1064158 \\
34324\end{array}$ & 2 & $\begin{array}{r}1413583 \\
216086\end{array}$ \\
\hline
\end{tabular}

1191720

1191720

38834

221900
$113392 \quad 1078328$

$295154 \quad 896566$

38834
1191720

1191720

38834

221900

2 


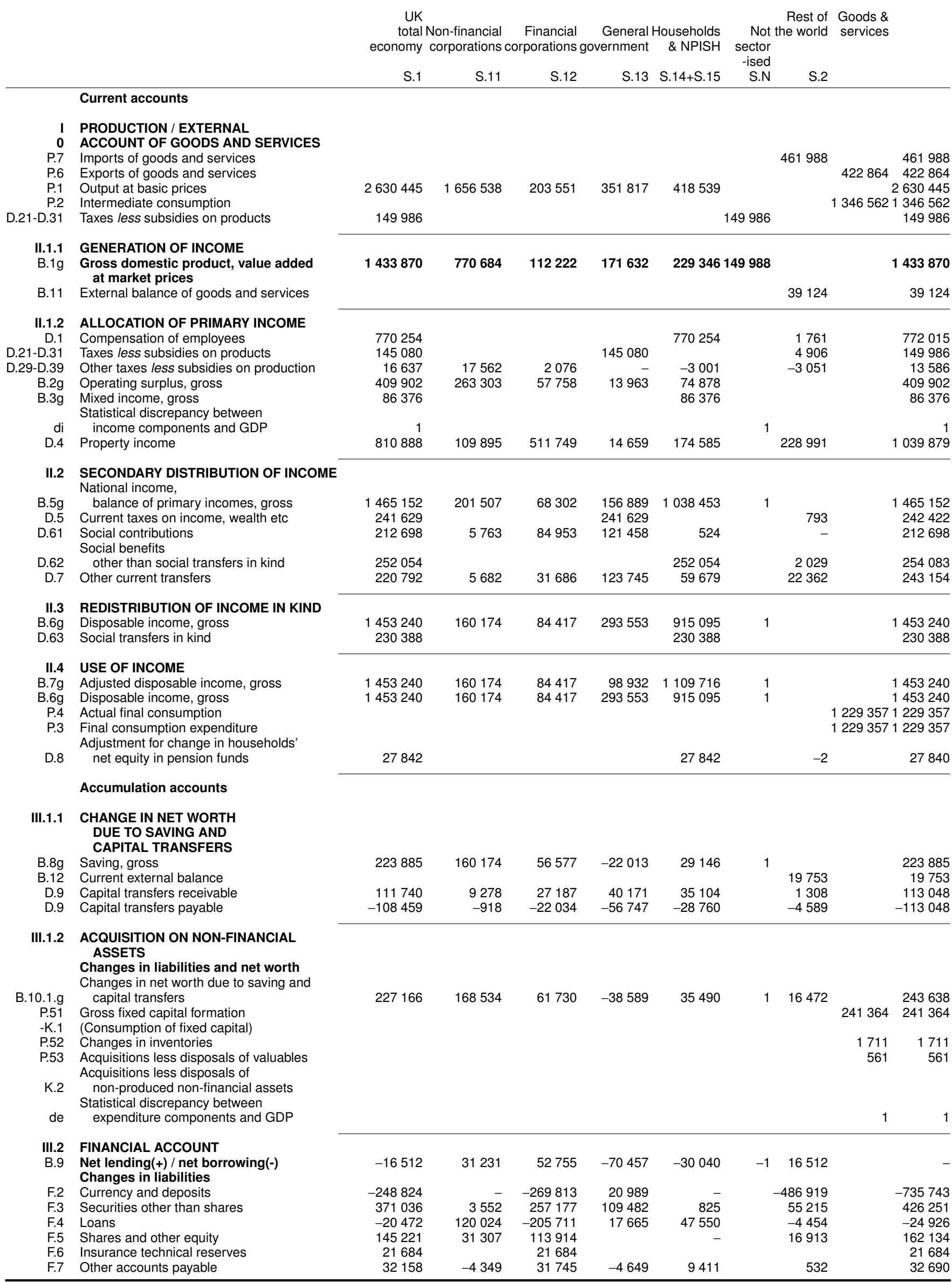


I PRODUCTION / EXTERNAL

0 ACCOUNT OF GOODS AND SERVICES

P.7 Imports of goods and services

P.6 Exports of goods and services

P.1 Output at basic prices

P.2 Intermediate consumption

D.21-D.31 Taxes less subsidies on products

B.1g Gross domestic product, value added at market prices

B.11 External balance of goods and services

II.1.1 GENERATION OF INCOME

D.1 Compensation of employees

D.21-D.31 Taxes less subsidies on products

D.29-D.39 Other taxes less subsidies on production

B.2g Operating surplus, gross

B.3g Mixed income, gross

Statistical discrepancy between

di income components and GDP

II.1.2 ALLOCATION OF PRIMARY INCOME

D.4 Property income

National income

B.5g balance of primary incomes, gross

II.2 SECONDARY DISTRIBUTION OF INCOME

D.5 Current taxes on income, wealth etc

D.61 Social contributions

Social benefits

D.62 other than social transfers in kind

D.7 Other current transfers

B.6g Disposable income, gross

II.3 REDISTRIBUTION OF INCOME IN KIND

B.7g Adjusted disposable income, gross

D.63 Social transfers in kind

II.4 USE OF INCOME

B.6g Disposable income, gross

P.4 Actual final consumption

P.3 Final consumption expenditure

Adjustment for change in households

D.8 net equity in pension funds

B.8g Saving, gross

B.12 Current external balance

$\begin{array}{lllll}1433870 & 770684 & 112222 & 171632 & 229346149988\end{array}$

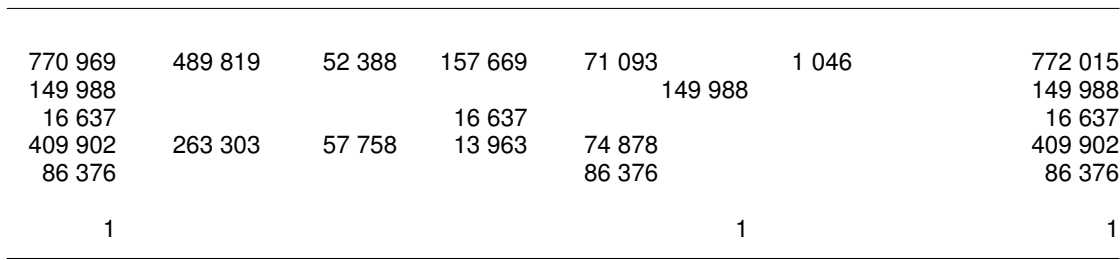

\begin{tabular}{|c|c|c|c|c|c|c|}
\hline 777037 & 171691 & 501205 & 36501 & 67640 & 262842 & 1039879 \\
\hline 1465152 & 201507 & 68302 & 156889 & 1038453 & 1 & 1465152 \\
\hline $\begin{array}{l}241806 \\
212630\end{array}$ & 40845 & 11645 & 1147 & $\begin{array}{l}188169 \\
212630\end{array}$ & $\begin{array}{r}616 \\
68\end{array}$ & $\begin{array}{l}242422 \\
212698\end{array}$ \\
\hline $\begin{array}{l}254083 \\
230566\end{array}$ & $\begin{array}{l}5763 \\
6170\end{array}$ & $\begin{array}{l}57113 \\
31766\end{array}$ & $\begin{array}{l}190187 \\
158834\end{array}$ & $\begin{array}{r}1020 \\
33796\end{array}$ & 12588 & $\begin{array}{l}254083 \\
243154\end{array}$ \\
\hline 1453240 & 160174 & 84417 & 293553 & 915095 & 1 & 145324 \\
\hline $\begin{array}{r}1453240 \\
230388\end{array}$ & 160174 & 84417 & $\begin{array}{r}98932 \\
194621\end{array}$ & $\begin{array}{r}1109716 \\
35767\end{array}$ & 1 & $\begin{array}{r}1453240 \\
230388\end{array}$ \\
\hline
\end{tabular}

\section{Accumulation accounts}

CHANGE IN NET WORTH

III.1.1 DUE TO SAVING AND CAPITAL TRANSFERS

D.9 Capital transfers receivable

D.9 Capital transfers payable
Changes in net worth d

B.10.1.g and capital transfers

\section{III.1.2 ACQUISITION OF NON-FINANCIAL ASSETS} Changes in assets

P.51 Gross fixed capital formation

-K.1 (Consumption of fixed capital)

P.52 Changes in inventories

P.53 Acquisitions less disposals of valuables Acquisitions less disposals of

K.2 non-produced non-financial assets Statistical discrepancy between

de expenditure components and GDP

B.9 Net lending(+) / net borrowing(-)

III.2 FINANCIAL ACCOUNT: changes in assets

F.1 Monetary gold and SDRs

F.2 Currency and deposits

F.3 Securities other than shares

F.4 Loans

F.5 Shares and other equity

F.6 Insurance technical reserves

F.7 Other accounts receivable

Statistical discrepancy between non-financial

\begin{tabular}{|c|c|c|c|c|c|c|c|}
\hline 227166 & 168534 & 61730 & -38589 & 35490 & 1 & 16472 & 243638 \\
\hline 241364 & 134071 & 8704 & 32860 & 65729 & & & 241364 \\
\hline-151370 & -86127 & -6366 & -13963 & -44914 & & & -151370 \\
\hline 1711 & 1744 & -17 & -41 & 25 & & & 1711 \\
\hline 561 & 150 & 284 & 11 & 116 & & & 561 \\
\hline 40 & 1338 & 4 & -962 & -340 & & -40 & - \\
\hline 1 & & & & & 1 & & 1 \\
\hline-16512 & 31231 & 52755 & -70457 & -30040 & -1 & 16512 & - \\
\hline-24 & & & -24 & & & 24 & \\
\hline-276341 & -30498 & -336784 & 31769 & 59172 & & -459402 & -735743 \\
\hline 259443 & 5093 & 251539 & 5659 & -2848 & & 166808 & 426251 \\
\hline 164376 & 100474 & 50246 & 7301 & 6355 & & -189302 & -24926 \\
\hline 69249 & 121151 & 21837 & 10866 & -84605 & & 92885 & 162134 \\
\hline 25600 & 318 & 33 & 21 & 25228 & & -3916 & 21684 \\
\hline 32515 & -5064 & 7345 & 16689 & 13545 & & 175 & 32690 \\
\hline 9473 & -9709 & 7535 & 749 & 10899 & -1 & -9473 & - \\
\hline
\end{tabular}


total Non-financial Financial General Households Not the world services economy corporations corporations government \& NPISH sector

$\begin{array}{lllllrl}\text { S.1 } & \text { S.11 } & \text { S.12 } & \text { S.13 } & \text { S.14+S.15 } & \text { S.N } & \text { S.2 }\end{array}$

I PRODUCTION / EXTERNAL

0 ACCOUNT OF GOODS AND SERVICES

P.7 Imports of goods and services

P.6 Exports of goods and services

P.1 Output at basic prices

P.2 Intermediate consumption

D.21-D.31 Taxes less subsidies on products

II.1.1 GENERATION OF INCOME

B.1g Gross domestic product, value added at market prices

B.11 External balance of goods and services

II.1.2 ALLOCATION OF PRIMARY INCOME

D. 1 Compensation of employees

D.21-D.31 Taxes less subsidies on products

D.29-D.39 Other taxes less subsidies on production

B.2g Operating surplus, gross

B.3g Mixed income, gross

Statistical discrepancy between

di income components and GDP

D.4 Property income

II.2 SECONDARY DISTRIBUTION OF INCOME National income

B.5g balance of primary incomes, gross

D.5 Current taxes on income, wealth etc

D.61 Social contributions

Social benefits

D.62 other than social transfers in kind

D.7 Other current transfers

II.3 REDISTRIBUTION OF INCOME IN KIND

B.6g Disposable income, gross

D.63 Social transfers in kind

II.4 USE OF INCOME

B.7g Adjusted disposable income, gross

B.6g Disposable income, gross

P.4 Actual final consumption

P.3 Final consumption expenditure

Adjustment for change in households

D.8 net equity in pension funds

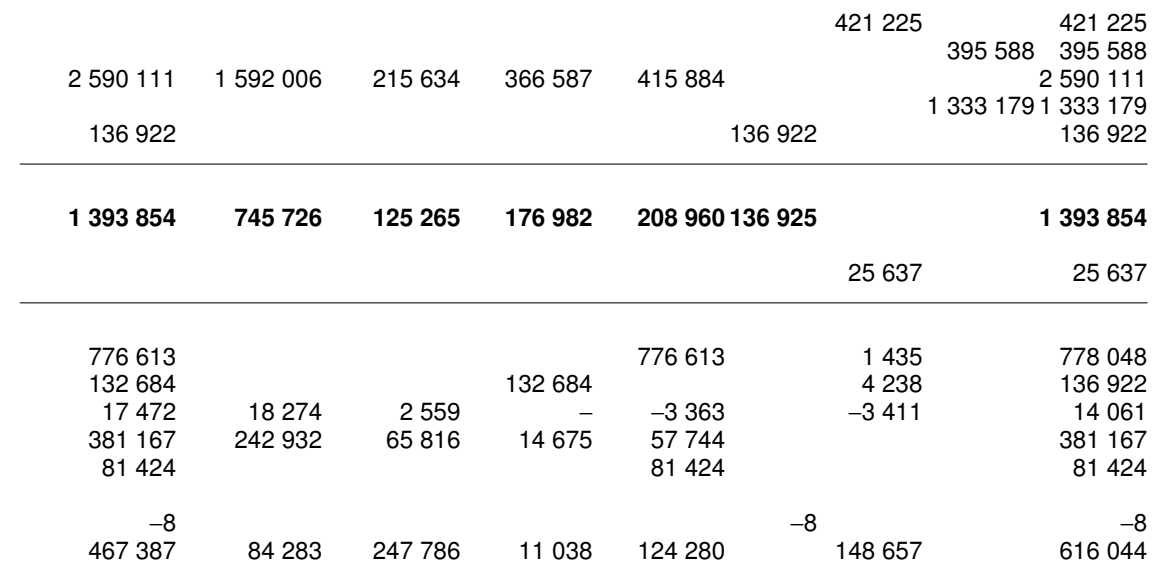

Accumulation accounts

III.1.1 CHANGE IN NET WORTH DUE TO SAVING

DUE TO SAVING AND CAPITAL TRANSFERS

B.8g Saving, gross

B.12 Current external balance

D.9 Capital transfers receivable

D.9 Capital transfers payable

III.1.2 ACQUISITION ON NON-FINANCIAL ASSETS

Changes in liabilities and net worth

Changes in net worth due to saving and

B.10.1.g capital transfers

P.51 Gross fixed capital formation

-K.1 (Consumption of fixed capital)

P.52 Changes in inventories

P.53 Acquisitions less disposals of valuables

Acquisitions less disposals of

K.2 non-produced non-financial assets

Statistical discrepancy between

de expenditure components and GDP

III.2 FINANCIAL ACCOUNT

B.9 Net lending(+) / net borrowing(-)

Changes in liabilities

F2 Currency and deposits

F.3 Securities other than shares

F.4 Loans

F.5 Shares and other equity

F.6 Insurance technical reserves

F.7 Other accounts payable

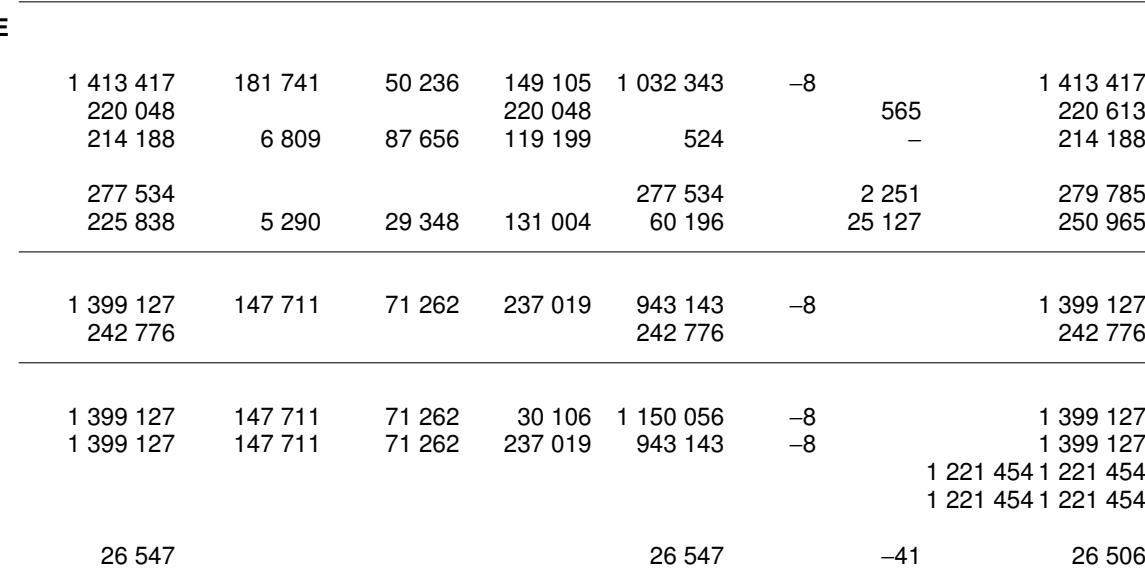


UK

total Non-financial Financial General Households Not the world services economy corporations corporations government \&NPISH sector

S. 1

S. 11 S.12

S.13 S.14+S.15 S.N S.2

\section{Current accounts}

I PRODUCTION / EXTERNAL

0 ACCOUNT OF GOODS AND SERVICES

P.7 Imports of goods and services

P.6 Exports of goods and services

P.1 Output at basic prices

P2 Intermediate consumption

D.21-D.31 Taxes less subsidies on products

B.1g Gross domestic product, value added at market prices

B.11 External balance of goods and services

II.1.1 GENERATION OF INCOME

D.1 Compensation of employees

D.21-D.31 Taxes less subsidies on products

D.29-D.39 Other taxes less subsidies on production

B.2g Operating surplus, gross

B.3g Mixed income, gross

Statistical discrepancy between

di income components and GDP

II.1.2 ALLOCATION OF PRIMARY INCOME

D.4 Property income

National income

B. $5 \mathrm{~g}$ balance of primary incomes, gross

II. SECONDARY DISTRIBUTION OF INCOME

D.5 Current taxes on income, wealth etc

D.61 Social contributions

Social benefits

D. other than social transfers in kind

D.7 Other current transfers

B.6g Disposable income, gross

II.3 REDISTRIBUTION OF INCOME IN KIND

B.7g Adjusted disposable income, gross

D.63 Social transfers in kind

II.4 USE OF INCOME

B.6g Disposable income, gross

P.4 Actual final consumption

P.3 Final consumption expenditure Adjustment for change in households

D.8 net equity in pension funds

B.8g Saving, gross

B.12 Current external balance

Accumulation accounts

CHANGE IN NET WORTH

III.1.1 DUE TO SAVING AND CAPITAL TRANSFERS

D.9 Capital transfers receivable

D.9 Capital transfers payable

Changes in net worth due to saving

B.10.1.g and capital transfers

III.1.2 ACQUISITION OF NON-FINANCIAL ASSETS

Changes in assets

P.51 Gross fixed capital formation

-K.1 (Consumption of fixed capital)

P.52 Changes in inventories

P.53 Acquisitions less disposals of valuables Acquisitions less disposals of

K.2 non-produced non-financial assets Statistical discrepancy between

de expenditure components and GDP

B.9 Net lending(+) / net borrowing(-)

III.2 FINANCIAL ACCOUNT: changes in assets

F.1 Monetary gold and SDRs

F.2 Currency and deposits

F.3 Securities other than shares

F.4 Loans

F.5 Shares and other equity

F.6 Insurance technical reserves

F.7 Other accounts receivable

Statistical discrepancy between non-financial

dB.9f and financial transactions

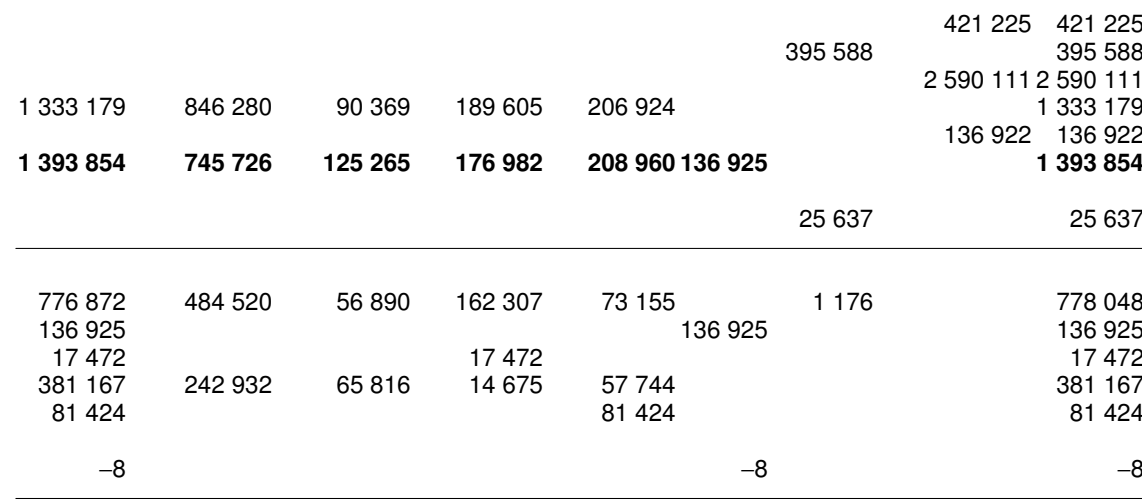

\begin{tabular}{rrrrrrrr}
\hline 446731 & 145474 & 263366 & 30173 & 7718 & 169313 & 616044 \\
1413417 & 181741 & 50236 & 149105 & 1032343 & -8 & 1413417
\end{tabular}

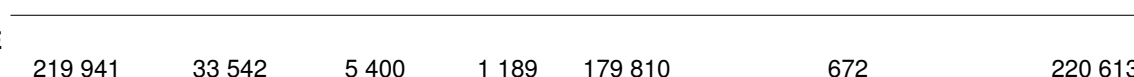

\begin{tabular}{|c|c|c|c|c|c|c|}
\hline $\begin{array}{l}219941 \\
214016\end{array}$ & 33542 & 5400 & 1189 & $\begin{array}{l}179810 \\
214016\end{array}$ & $\begin{array}{l}672 \\
172\end{array}$ & $\begin{array}{l}220613 \\
214188\end{array}$ \\
\hline $\begin{array}{r}279785 \\
238156 \\
1399127\end{array}$ & $\begin{array}{r}6809 \\
5778 \\
147711\end{array}$ & $\begin{array}{ll}61 & 150 \\
29 & 428 \\
71 & 262\end{array}$ & $\begin{array}{l}210806 \\
170342 \\
237019\end{array}$ & $\begin{array}{r}1020 \\
32608 \\
943143\end{array}$ & 12809 & $\begin{array}{r}279785 \\
250965 \\
1399127\end{array}$ \\
\hline $\begin{array}{r}1399127 \\
242776\end{array}$ & 147711 & 71262 & $\begin{array}{r}30106 \\
206913\end{array}$ & $\begin{array}{r}1150056 \\
35863\end{array}$ & -8 & $\begin{array}{r}1399127 \\
242776\end{array}$ \\
\hline
\end{tabular}

1221454

1221454

26506

26506
177714
$120436 \quad 1101018$

327349894105

26506
1221454

1221454

26506

177714
180978

$52895-115813$

83788

$-8 \quad 17052$

198030

\begin{tabular}{|c|c|c|c|c|c|c|c|}
\hline $\begin{array}{r}209253 \\
-159862 \\
-11651 \\
429\end{array}$ & $\begin{array}{r}119496 \\
-90888 \\
-11391 \\
153\end{array}$ & $\begin{array}{r}6230 \\
-6924 \\
53 \\
264\end{array}$ & $\begin{array}{r}37125 \\
-14675 \\
36 \\
12\end{array}$ & $\begin{array}{r}46401 \\
-47375 \\
-349 \\
-\end{array}$ & & & $\begin{array}{r}209253 \\
-159862 \\
-11651 \\
429\end{array}$ \\
\hline-373 & 978 & 16 & -1019 & -348 & & 373 & - \\
\hline & & & & & - & & - \\
\hline$-166 / 9$ & 50880 & 46332 & -151968 & 38084 & -8 & $166 / 9$ & - \\
\hline
\end{tabular}

\begin{tabular}{rrrrrrr}
-132 & & & & 132 & 132 \\
383511 & -33934 & 392824 & 9674 & 14947 & -323938 & 59573 \\
354494 & -6138 & 355515 & 1883 & 3234 & 139486 & -993980 \\
-275945 & -46465 & -232060 & 4472 & -1892 & -97701 & -373646 \\
162292 & 24132 & 87240 & 37352 & 13568 & 93670 & 255962 \\
18778 & -354 & -37 & -24 & 19193 & -323 & 18455 \\
-6514 & -5635 & 6176 & 403 & -7458 & 108 & -6406 \\
8086 & -1378 & 851 & -697 & 9318 & -8 & -8086 \\
\hline
\end{tabular}


totalNon-financial Financial GeneralHouseholds Notthe world services economy corporationscorporationsgovernment \& NPISH sector

$\begin{array}{lllllrl}\text { S.1 } & \text { S.11 } & \text { S.12 } & \text { S.13 } & \text { S.14+S.15 } & \text { S.N } & \text { S.2 }\end{array}$

Current accounts

I PRODUCTION / EXTERNAL

0 ACCOUNT OF GOODS AND SERVICES

P.7 Imports of goods and services

P.6 Exports of goods and services

P.1 Output at basic prices

P.2 Intermediate consumption

D.21-D.31 Taxes less subsidies on products

II.1.1 GENERATION OF INCOME

B.1g Gross domestic product, value added at market prices

B.11 External balance of goods and services

II.1.2 ALLOCATION OF PRIMARY INCOME

D.1 Compensation of employees

D.21-D.31 Taxes less subsidies on products

D.29-D.39 Other taxes less subsidies on production

B.2g Operating surplus, gross

B.3g Mixed income, gross

Statistical discrepancy between

di income components and GDP

D.4 Property income

II.2 SECONDARY DISTRIBUTION OF INCOME National income,

B.5g balance of primary incomes, gross

D.5 Current taxes on income, wealth etc

D.61 Social contributions

Social benefits

D.62 other than social transfers in kind

D.7 Other current transfers

II.3 REDISTRIBUTION OF INCOME IN KIND

B.6g Disposable income, gross

D.63 Social transfers in kind

II.4 USE OF INCOME

B.7g Adjusted disposable income, gross

B.6g Disposable income, gross

P.4 Actual final consumption

P.3 Final consumption expenditure Adjustment for change in households' net equity

D.8 in pension funds

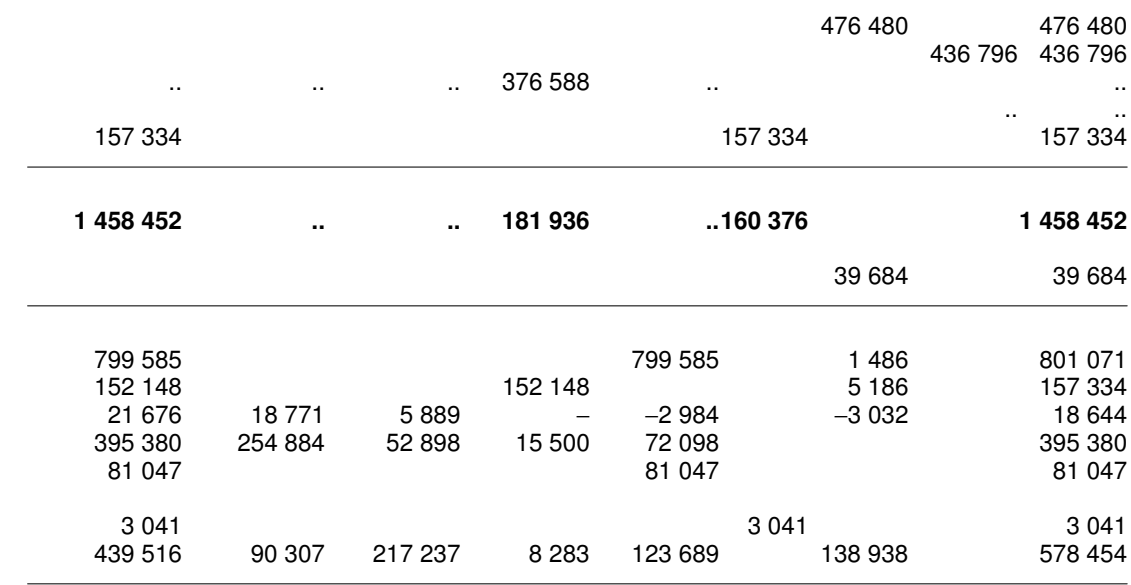

Accumulation accounts

\section{III.1.1 CHANGE IN NET WORTH DUE TO SAVING}

\section{AND CAPITAL TRANSFERS}

B.8g Saving, gross

B.12 Current external balance

D.9 Capital transfers receivable

D.9 Capital transfers payable

III.1.2 ACQUISITION ON NON-FINANCIAL ASSETS

Changes in liabilities and net worth

Changes in net worth due to saving and

B.10.1.g capital transfers

P.51 Gross fixed capital formation

-K.1 (Consumption of fixed capital)

P.52 Changes in inventories

P.53 Acquisitions less disposals of valuables

Acquisitions less disposals of

K.2 non-produced non-financial assets

Statistical discrepancy between

de expenditure components and GDP

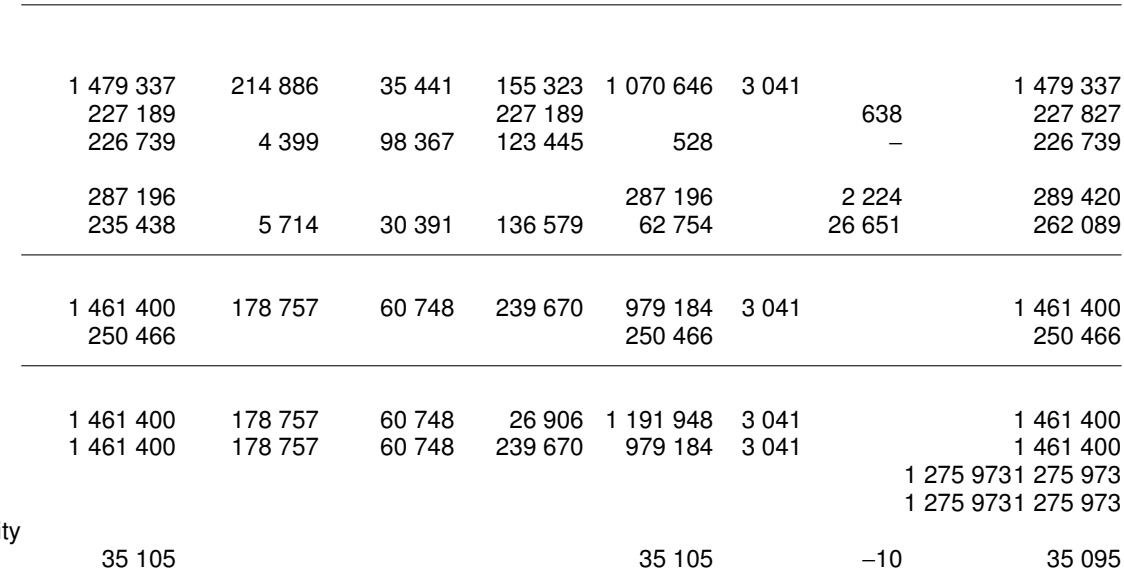

\section{III.2 FINANCIAL ACCOUNT}

B.9 Net lending(+)/ net borrowing(-) Changes in liabilities

F.2 Currency and deposits

F.3 Securities other than shares

F.4 Loans

F.5 Shares and other equity

F.6 Insurance technical reserves

F.7 Other accounts payable

\begin{tabular}{rrrrrrr}
185437 & 178757 & 25653 & -98397 & 76383 & 3041 & \\
& & & & & 36726 & 185437 \\
39958 & 10689 & 67 & 16152 & 13050 & 1077 & 41035 \\
-36307 & -456 & -67 & -32297 & -3487 & -4728 & -41035 \\
\hline
\end{tabular}

$\begin{array}{llllllll}189088 & 188990 & 25653 & -114542 & 85946 & 3041 & 33075 & 222163\end{array}$ $217108 \quad 217108$

64670
23392

-33018
31281
115521
157709
64670
23392
14326

61215

$-\quad 39272$

$-2618$

26368
18816

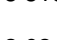

$-49995$

109768

45854

23392

2632
$32757 \quad 5455 \quad 33018$

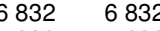

$638 \quad 638$

$-2415 \quad-2415$ 


\section{Current accounts}

I PRODUCTION / EXTERNAL

0 ACCOUNT OF GOODS AND SERVICES

P7 Imports of goods and services

P.6 Exports of goods and services

P.1 Output at basic prices

P.2 Intermediate consumption

D.21-D.31 Taxes less subsidies on products

B.1g Gross domestic product, value added at market prices

B.11 External balance of goods and services

II.1.1 GENERATION OF INCOME

D.1 Compensation of employees

D.21-D.31 Taxes less subsidies on products

D.29-D.39 Other taxes less subsidies on production

B.2g Operating surplus, gross

B.3g Mixed income, gross

Statistical discrepancy between

di income components and GDP

II.1.2 ALLOCATION OF PRIMARY INCOME

D.4 Property income

National income

B.5g balance of primary incomes, gross

II. SECONDARY DISTRIBUTION OF INCOME

D.5 Current taxes on income, wealth etc

D.61 Social contributions

Social benefits

D.62 other than social transfers in kind

D.7 Other current transfers

B.6g Disposable income, gross

II.3 REDISTRIBUTION OF INCOME IN KIND

B.7g Adjusted disposable income, gross

D.63 Social transfers in kind

II.4 USE OF INCOME

B.6g Disposable income, gross

P.4 Actual final consumption

P.3 Final consumption expenditur Adjustment for change in households

D.8 net equity in pension funds

B.8g Saving, gross

B.12 Current external balance

Accumulation accounts

CHANGE IN NET WORTH DUE TO SAVING

III.1.1 AND CAPITAL TRANSFERS

D.9 Capital transfers receivable

D.9 Capital transfers payable

Changes in net worth due to saving

B.10.1.g and capital transfers

\begin{tabular}{|c|c|c|c|c|c|c|c|c|}
\hline & & & & & & 436796 & 476480 & $\begin{array}{l}476480 \\
436796\end{array}$ \\
\hline .. & .. & .. & & .. & & & & \\
\hline 1458452 & .. & .. & 181936 & & 160376 & & 0004 & $\begin{array}{r}158 \\
14584\end{array}$ \\
\hline & & & & & & 39684 & & 39684 \\
\hline $\begin{array}{l}799974 \\
157334\end{array}$ & 499091 & 58805 & 166436 & 75642 & 157334 & 1097 & & $\begin{array}{l}801071 \\
157334\end{array}$ \\
\hline 21676 & & & 21676 & & & & & 21676 \\
\hline $\begin{array}{r}395380 \\
81047\end{array}$ & 254884 & 52898 & 15500 & $\begin{array}{l}72098 \\
81047\end{array}$ & & & & $\begin{array}{r}395380 \\
81047\end{array}$ \\
\hline 3041 & & & & & 3041 & & & 30 \\
\hline 416088 & 130305 & 234694 & 45316 & 5773 & & 162366 & & 578454 \\
\hline 1479337 & 214886 & 35441 & 155323 & 1070646 & 3041 & & & 1479337 \\
\hline $\begin{array}{l}227260 \\
226656\end{array}$ & 35641 & 9708 & 1236 & $\begin{array}{l}180675 \\
226656\end{array}$ & & $\begin{array}{r}567 \\
83\end{array}$ & & $\begin{array}{l}227827 \\
226739\end{array}$ \\
\hline 289420 & 4399 & 63272 & 220725 & 1024 & & & & 289420 \\
\hline 251163 & 6202 & 30471 & 180905 & 33585 & & 10926 & & $\begin{array}{r}262089 \\
1461400\end{array}$ \\
\hline 1461400 & 178757 & 60748 & 239670 & 979184 & 3041 & & & 1461400 \\
\hline $\begin{array}{r}1461400 \\
250466\end{array}$ & 178757 & 60748 & $\begin{array}{r}26906 \\
212764\end{array}$ & $\begin{array}{r}1191948 \\
37702\end{array}$ & 3041 & & & $\begin{array}{r}1461400 \\
250466\end{array}$ \\
\hline
\end{tabular}

1275973

1275973

$125303 \quad 1150670$

$338067 \quad 937906$

1275973

35095

185437

178757
1275973

35095

185437 36726

\section{III.1.2 ACQUISITION OF NON-FINANCIAL ASSETS}

Changes in assets

P.51 Gross fixed capital formation

-K.1 (Consumption of fixed capital)

P.52 Changes in inventories

P.53 Acquisitions less disposals of valuables

Acquisitions less disposals of

K.2 non-produced non-financial assets

Statistical discrepancy between

de expenditure components and GDP

B.9 Net lending(+) / net borrowing(-)

III.2 FINANCIAL ACCOUNT: changes in assets

F.1 Monetary gold and SDRs

F.2 Currency and deposits

F.3 Securities other than shares

F.4 Loans

F.5 Shares and other equity

F.6 Insurance technical reserves

F.7 Other accounts receivable

Statistical discrepancy between non-financial

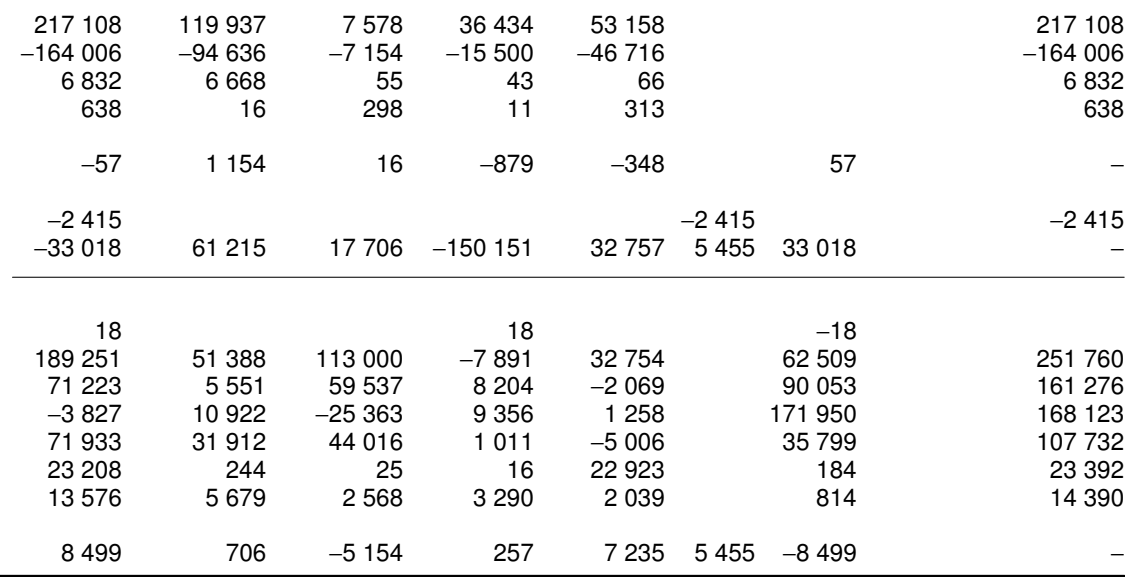




\begin{tabular}{|c|c|c|c|c|c|c|c|}
\hline & & $\begin{array}{r}\text { UK } \\
\text { total economy } \\
\text { S.1 }\end{array}$ & $\begin{array}{r}\text { Non-financial } \\
\text { corporations } \\
\text { S. } 11\end{array}$ & $\begin{array}{r}\text { Financial } \\
\text { corporations } \\
\text { S.12 }\end{array}$ & $\begin{array}{r}\text { Monetary } \\
\text { financial } \\
\text { institutions } \\
\text { S.121+S.122 }\end{array}$ & $\begin{array}{r}\text { Other financial } \\
\text { intermediaries } \\
\& \text { auxiliaries } \\
\text { S.123+S.124 }\end{array}$ & $\begin{array}{r}\text { Insurance } \\
\text { corporations } \\
\text { \& pension funds } \\
\mathrm{S} .125\end{array}$ \\
\hline $\mathbf{I}$ & PRODUCTION ACCOUNT & & & & & & \\
\hline & Resources & & & & & & \\
\hline P.1 & Output & & & & & & \\
\hline $\begin{array}{l}\text { P.12 } \\
\text { P.13 }\end{array}$ & $\begin{array}{l}\text { Output for own final use } \\
\text { Other non-market output }\end{array}$ & $\begin{array}{l}119347 \\
363212\end{array}$ & 17446 & 3011 & & & \\
\hline P.1 & Total output & 2590111 & 1592006 & 215634 & & & \\
\hline $\begin{array}{r}\text { D.21 } \\
-D .31\end{array}$ & $\begin{array}{l}\text { Taxes on products } \\
\text { less Subsidies on products }\end{array}$ & $\begin{array}{r}142629 \\
-5707\end{array}$ & & & & & \\
\hline Total & Total resources & 2727033 & 1592006 & 215634 & & & \\
\hline & Uses & & & & & & \\
\hline P.2 & Intermediate consumption & 1333179 & 846280 & 90369 & & & \\
\hline B. $1^{*} \mathrm{~g}$ & Gross domestic product & 1393854 & 745726 & 125265 & 73058 & 34289 & 17918 \\
\hline Total & Total uses & 2727033 & 1592006 & 215634 & & & \\
\hline $\begin{array}{r}\text { B. } 1^{*} \mathbf{g} \\
-K .1\end{array}$ & $\begin{array}{l}\text { Gross domestic product } \\
\text { less Fixed capital consumption }\end{array}$ & $\begin{array}{r}1393854 \\
-159862\end{array}$ & $\begin{array}{l}745726 \\
-90888\end{array}$ & $\begin{array}{r}125265 \\
-6924\end{array}$ & 73058 & 34289 & 17918 \\
\hline B. $1^{*} n$ & Net domestic product & 1233992 & 654838 & 118341 & & & \\
\hline
\end{tabular}

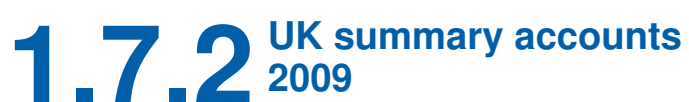

Total economy: all sectors and the rest of the world

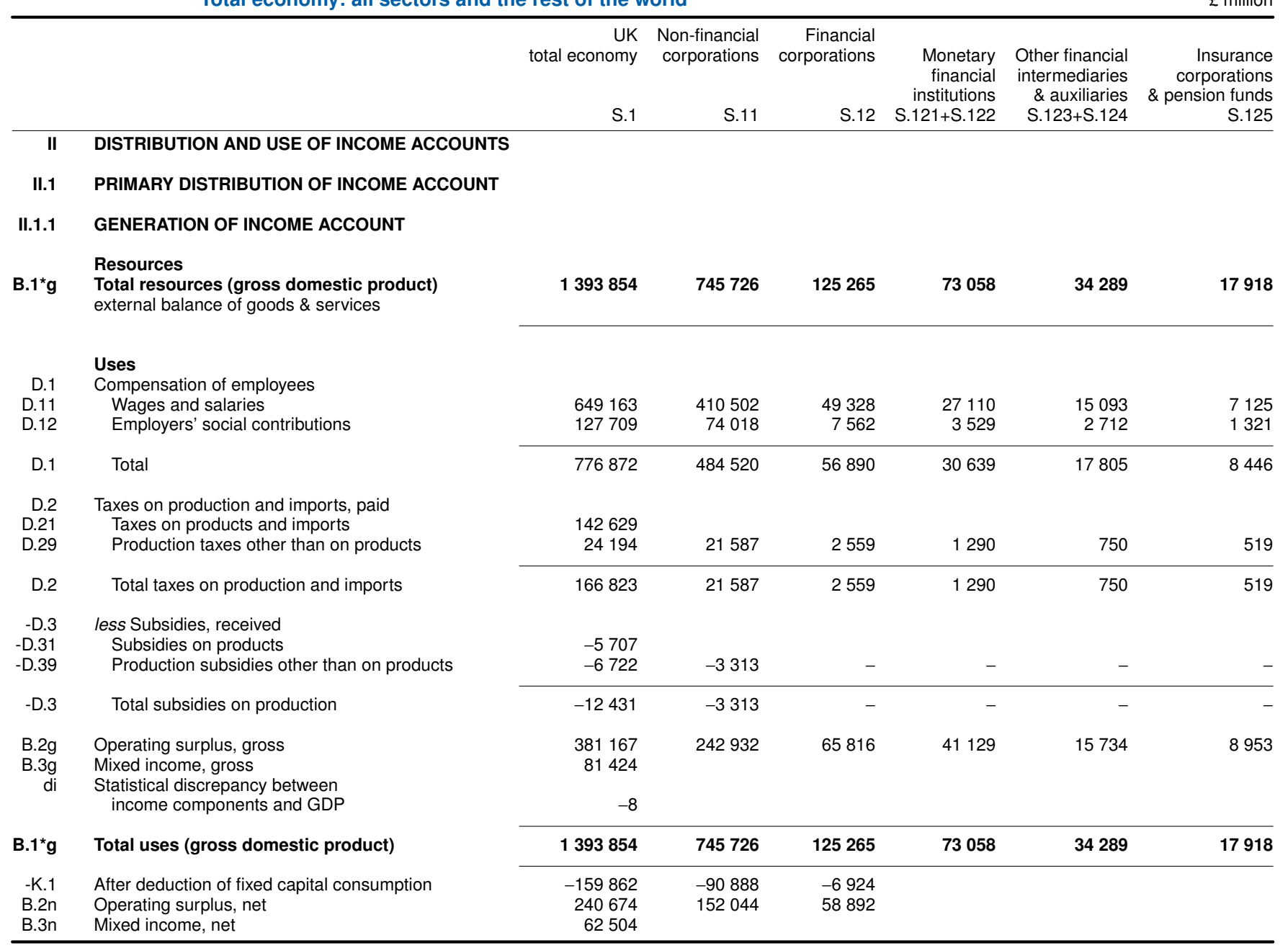




\begin{tabular}{|c|c|c|c|c|c|c|c|c|}
\hline continued & Total economy: al & ctors and tr & est of the w & & & & & $£$ million \\
\hline & & $\begin{array}{r}\text { General } \\
\text { government }\end{array}$ & $\begin{array}{r}\text { Central } \\
\text { government }\end{array}$ & $\begin{array}{r}\text { Local } \\
\text { government }\end{array}$ & $\begin{array}{l}\text { Households } \\
\text { \& NPISH }\end{array}$ & $\begin{array}{r}\text { Not } \\
\text { sector } \\
\text {-ised }\end{array}$ & & $\begin{array}{r}\text { Rest of } \\
\text { the world }\end{array}$ \\
\hline & & S.13 & S.1311 & S.1313 & S.14+S.15 & S.N & $\begin{array}{c}\text { Taxes less } \\
\text { subsidies }\end{array}$ & S.2 \\
\hline \multirow[t]{2}{*}{ I } & PRODUCTION ACCOUNT & & & & & & & \\
\hline & Resources & & & & & & & \\
\hline P.1 & Output & & & & & & & \\
\hline P.11 & Market output ${ }^{\star}$ & 38834 & 13420 & 25414 & 281535 & & & \\
\hline P.12 & Output for own final use & 404 & & 348 & 98486 & & & \\
\hline P.13 & Other non-market output & 327349 & 199649 & 127700 & 35863 & & & \\
\hline P.1 & Total output & 366587 & 213125 & 153462 & 415884 & & & \\
\hline $\begin{array}{r}\text { D.21 } \\
-D .31\end{array}$ & $\begin{array}{l}\text { Taxes on products } \\
\text { less Subsidies on products }\end{array}$ & & & & & $\begin{array}{r}142629 \\
-5707\end{array}$ & $\begin{array}{r}142629 \\
-5707\end{array}$ & \\
\hline \multirow[t]{2}{*}{ Total } & Total resources & 366587 & 213125 & 153462 & 415884 & 136922 & 136922 & \\
\hline & Uses & & & & & & & \\
\hline P.2 & \multirow{2}{*}{$\begin{array}{l}\text { Intermediate consumption } \\
\text { Gross domestic product }\end{array}$} & 189605 & 118188 & 71417 & 206924 & & & \\
\hline B. $1^{\star} \mathrm{g}$ & & 176982 & 94937 & 82045 & 208960 & 136925 & 136922 & \\
\hline Total & Total uses & 366587 & 213125 & 153462 & 415884 & 136922 & 136922 & \\
\hline $\begin{aligned} \text { B. } 1^{*} \mathbf{g} \\
-K .1\end{aligned}$ & $\begin{array}{l}\text { Gross domestic product } \\
\text { less Fixed capital consumption }\end{array}$ & $\begin{array}{r}176982 \\
-14675\end{array}$ & $\begin{array}{l}94937 \\
-7232\end{array}$ & $\begin{array}{l}82045 \\
-7443\end{array}$ & $\begin{array}{l}208960 \\
-47375\end{array}$ & 136925 & 136922 & \\
\hline B. $1^{*} n$ & Net domestic product & 162307 & 87705 & 74602 & 161585 & 136922 & 136922 & \\
\hline
\end{tabular}

\subsubsection{9 summary accounts

\begin{tabular}{|c|c|c|c|c|c|}
\hline $\begin{array}{r}\text { General } \\
\text { government }\end{array}$ & $\begin{array}{r}\text { Central } \\
\text { government }\end{array}$ & $\begin{array}{r}\text { Local } \\
\text { government }\end{array}$ & $\begin{array}{r}\text { Households } \\
\text { \& NPISH }\end{array}$ & $\begin{array}{r}\text { Not } \\
\text { sector } \\
\text {-ised }\end{array}$ & \\
\hline S.13 & S.1311 & S.1313 & S.14+S.15 & S.N & subsidies \\
\hline
\end{tabular}

\begin{tabular}{|c|c|c|c|c|c|c|c|c|}
\hline & & S.13 & S.1311 & S.1313 & S.14+S.15 & S.N & subsidies & S.2 \\
\hline II & DISTRIBUTION AND USE OF INCOME ACCOUNTS & & & & & & & \\
\hline II.1 & PRIMARY DISTRIBUTION OF INCOME ACCOUNT & & & & & & & \\
\hline B. $1^{*} \mathrm{~g}$ & $\begin{array}{l}\text { Resources } \\
\text { Total resources (gross domestic product) } \\
\text { external balance of goods \& services }\end{array}$ & 176982 & 94937 & 82045 & 208960 & 136925 & 136922 & 25637 \\
\hline $\begin{array}{r}\text { D.1 } \\
\text { D. } 11 \\
\text { D. } 12\end{array}$ & $\begin{array}{l}\text { Uses } \\
\text { Compensation of employees } \\
\text { Wages and salaries } \\
\text { Employers' social contributions }\end{array}$ & $\begin{array}{r}127294 \\
35013\end{array}$ & $\begin{array}{l}70165 \\
17540\end{array}$ & $\begin{array}{l}57129 \\
17473\end{array}$ & $\begin{array}{l}62039 \\
11116\end{array}$ & & & 1176 \\
\hline D.1 & Total & 162307 & 87705 & 74602 & 73155 & & & 1176 \\
\hline $\begin{array}{r}\text { D.2 } \\
\text { D. } 21 \\
\text { D.29 }\end{array}$ & $\begin{array}{l}\text { Taxes on production and imports, paid } \\
\text { Taxes on products and imports } \\
\text { Production taxes other than on products }\end{array}$ & - & - & - & 48 & 142629 & 142629 & - \\
\hline D.2 & Total taxes on production and imports & - & - & - & 48 & 142629 & 142629 & - \\
\hline $\begin{array}{r}-D .3 \\
-D .31 \\
-D .39\end{array}$ & $\begin{array}{l}\text { less Subsidies, received } \\
\text { Subsidies on products } \\
\text { Production subsidies other than on products }\end{array}$ & - & - & - & -3411 & -5707 & -5707 & \\
\hline$-\mathrm{D} .3$ & Total subsidies on production & - & - & - & -3411 & -5707 & -5707 & \\
\hline $\begin{array}{l}\text { B.2g } \\
\text { B. } 3 g \\
\text { di }\end{array}$ & $\begin{array}{l}\text { Operating surplus, gross } \\
\text { Mixed income, gross } \\
\text { Statistical discrepancy between } \\
\text { income components and GDP }\end{array}$ & 14675 & 7232 & 7443 & $\begin{array}{l}57744 \\
81424\end{array}$ & -8 & & \\
\hline B. $1^{*} \mathrm{~g}$ & Total uses (gross domestic product) & 176982 & 94937 & 82045 & 208960 & 136925 & 136922 & \\
\hline $\begin{array}{l}-K .1 \\
\text { B.2n } \\
\text { B.3n }\end{array}$ & $\begin{array}{l}\text { After deduction of fixed capital consumption } \\
\text { Operating surplus, net } \\
\text { Mixed income, net }\end{array}$ & $\begin{array}{r}-14675 \\
-\end{array}$ & $\begin{array}{r}-7232 \\
-\end{array}$ & $\begin{array}{r}-7443 \\
-\end{array}$ & $\begin{array}{r}-47375 \\
29738 \\
62504\end{array}$ & & & \\
\hline
\end{tabular}




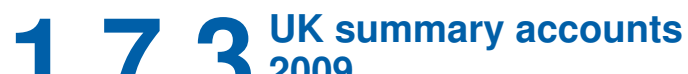

\begin{tabular}{|c|c|c|c|c|c|c|c|}
\hline & & $\begin{array}{r}\text { UK } \\
\text { total economy } \\
\text { S.1 }\end{array}$ & $\begin{array}{l}\text { Non-financial } \\
\text { corporations }\end{array}$ & $\begin{array}{r}\text { Financial } \\
\text { corporations } \\
\text { S. } 12\end{array}$ & $\begin{array}{r}\text { Monetary } \\
\text { financial } \\
\text { institutions } \\
\text { S.121+S.122 }\end{array}$ & $\begin{array}{r}\text { Other financial } \\
\text { intermediaries } \\
\& \text { auxiliaries } \\
\text { S.123+S.124 }\end{array}$ & $\begin{array}{r}\text { Insurance } \\
\text { corporations } \\
\text { \& pension funds } \\
\mathrm{S} .125\end{array}$ \\
\hline \multirow[t]{2}{*}{ II.1.2 } & ALLOCATION OF PRIMARY INCOME ACCOUNT & & & & & & \\
\hline & Resources & & & & & & \\
\hline B.2g & Operating surplus, gross & 381167 & 242932 & 65816 & 41129 & 15734 & 8953 \\
\hline D.11 & Wages and salaries & 648904 & & & & & \\
\hline D.12 & Employers' social contributions & 127709 & & & & & \\
\hline \multirow{2}{*}{$\begin{array}{r}\text { D. } 1 \\
\text { di }\end{array}$} & Total & 776613 & & & & & \\
\hline & $\begin{array}{l}\text { Statistical discrepancy between } \\
\text { income components and GDP }\end{array}$ & -8 & & & & & \\
\hline D.2 & Taxes on production and imports, received & & & & & & \\
\hline D.212 & Taxes and duties on imports excluding VAT & - & & & & & \\
\hline D.2121 & Import duties & - & & & & & \\
\hline D.2122 & Taxes on imports excluding VAT and import duties & - & & & & & \\
\hline D.214 & Taxes on products excluding VAT and import duties & 60084 & & & & & \\
\hline D.21 & Total taxes on products & 138391 & & & & & \\
\hline D.29 & Other taxes on production & 24194 & & & & & \\
\hline D.2 & Total taxes on production and imports, received & 162585 & & & & & \\
\hline -D.3 & less Subsidies, paid & & & & & & \\
\hline$-D .31$ & Subsidies on products & -5707 & & & & & \\
\hline$-\mathrm{D} .39$ & Other subsidies on production & -3313 & & & & & \\
\hline$-\mathrm{D} .3$ & Total subsidies & -9020 & & & & & \\
\hline D.4 & Property income, received & & & & & & \\
\hline D. 41 & Interest & 209477 & 7399 & 184680 & 122304 & 35846 & 26530 \\
\hline D.42 & Distributed income of corporations & 176418 & 62606 & 63579 & 6796 & 29502 & 27281 \\
\hline D.4 & Total property income & 467387 & 84283 & 247786 & 128278 & 66917 & 52591 \\
\hline \multirow[t]{2}{*}{ Total } & Total resources & 1860148 & 327215 & 313602 & 169407 & 82651 & 61544 \\
\hline & Uses & & & & & & \\
\hline D.4 & Property income, paid & & & & & & \\
\hline D. 41 & Interest & 231165 & 31877 & 161636 & 111305 & 48949 & 1382 \\
\hline D.42 & Distributed income of corporations & 142385 & 113829 & 28556 & 1990 & 22329 & 4237 \\
\hline D.43 & Reinvested earnings on direct foreign investment & 4148 & -1445 & 5593 & -1075 & 6535 & 133 \\
\hline D.44 & Property income attributed to insurance policy holders & 67581 & & 67581 & & & 67581 \\
\hline D.45 & Rent & 1452 & 1213 & - & - & - & - \\
\hline D.4 & Total property income & 446731 & 145474 & 263366 & 112220 & 77813 & 73333 \\
\hline B. $5^{\star} \mathrm{g}$ & Gross national income (GNI) & 1413417 & 181741 & 50236 & 57187 & 4838 & -11789 \\
\hline Total & Total uses & 1860148 & 327215 & 313602 & 169407 & 82651 & 61544 \\
\hline$-\mathrm{K} .1$ & After deduction of fixed capital consumption & -159862 & -90888 & -6924 & & & \\
\hline B. $5^{\star} n$ & National income, net & 1253555 & 90853 & 43312 & & & \\
\hline
\end{tabular}




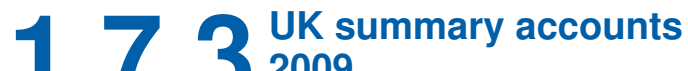

$\begin{array}{rr}\text { Central } & \text { Local } \\ \text { government } & \text { government }\end{array}$

\begin{tabular}{rrr} 
& £ million \\
\hline Households & $\begin{array}{r}\text { Not } \\
\text { sector } \\
\text {-ised }\end{array}$ & $\begin{array}{r}\text { Rest of } \\
\text { the world }\end{array}$
\end{tabular}

S.13

S.1311

S.1313

S.14+S.15

S.N

S.2

\section{II.1.2 ALLOCATION OF PRIMARY INCOME ACCOUNT}

\section{Resources}

Operating surplus, gross

Mixed income, gross

Compensation of employees

Wages and salaries

Employers' social contributions

Total

Statistical discrepancy between

income components and GDP

\begin{tabular}{rrrrr}
14675 & 7232 & 7443 & 57744 & \\
& & & 81424 & \\
& & & 648904 & 1435 \\
& & & 127709 & 1435 \\
\hline 78307 & 78307 & & & -8 \\
\\
\end{tabular}

Taxes on production and imports, received

Taxes on products

Value added tax (VAT)

Taxes and duties on imports excluding VAT Import duties

Taxes on imports excluding VAT and import duties

Taxes on products excluding VAT and import duties

Total taxes on products

Other taxes on production

Total taxes on production and imports, received

less Subsidies, paid

Subsidies on products

Other subsidies on production

Total subsidies

Property income, received

Interest

Distributed income of corporations

Reinvested earnings on direct foreign investment

Property income attributed to insurance policy holders

Rent

Total property income

Total resources

Uses

Property income, paid

Interest

Distributed income of corporations

Reinvested earnings on direct foreign investment

Property income attributed to insurance policy holders

Rent

30173

26948

3225

7479

84537

71535

13241

239

\begin{tabular}{rrrrrr}
\hline 30173 & 26948 & 3225 & 7718 & & 169313 \\
149105 & $\mathbf{1 4 6 6 1 2}$ & $\mathbf{2 4 9 3}$ & $\mathbf{1 0 3 2} \mathbf{3 4 3}$ & -8 & \\
\hline 179278 & 173560 & 5718 & 1040061 & -8 & \\
\hline-14675 & -7232 & -7443 & -47375 & & \\
134430 & 139380 & -4950 & 984968 & -8 &
\end{tabular}

Total property income

Gross national income (GNI)

Total uses

After deduction of fixed capital consumption

National income, net 


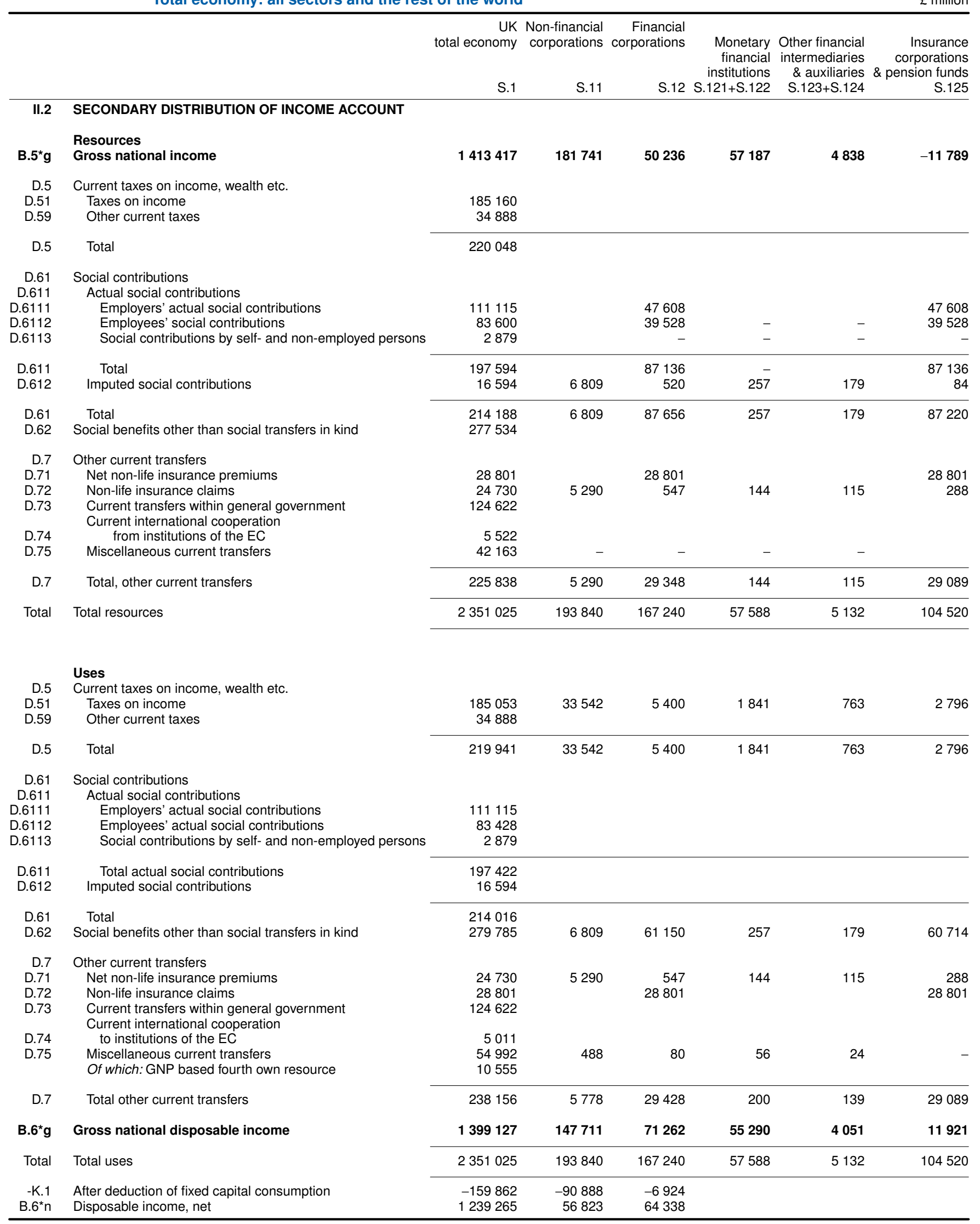




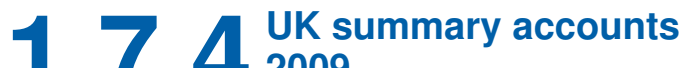

$\begin{array}{rrrrr}\text { Central } & \text { Local } & \begin{array}{r}\text { Households } \\ \text { \& NPISH }\end{array} & \begin{array}{r}\text { Not } \\ \text { sector } \\ \text {-ised }\end{array} & \begin{array}{r}\text { Rest of } \\ \text { the world }\end{array} \\ \text { government } & \text { government } & & \end{array}$

S.13

S.1311

S. 1313

S.14+S.15

S.N

\section{II.2 \\ SECONDARY DISTRIBUTION OF INCOME ACCOUNT}

B. $5^{\star} \mathrm{g}$

D.5

D.51

D.59

D.5

D.61

D.611

D.6111

D.6112

D.6113

D.611

D.612

D.61

D.62

D.7

D.71

D.72

D.73

D.74

D.75

D.7

Total

D.5

D.51

D.59

D.5

D.61

D.611

D.6111

D.6112

D. 6113

D.611

D.612

D.61

D.62

D.7

D.71

D.72

D.73

D.74

D.75

D.7

B. $6^{\star} \mathrm{g}$

Total

$-\mathrm{K} .1$
$\mathrm{~B} .6^{*} \mathrm{n}$
Resources

Gross national income

Current taxes on income, wealth etc.

Taxes on income

Other current taxes

Total

Social contributions

Actual social contributions

Employers' actual social contributions

Employees' social contributions

Social contributions by self- and non-employed persons

Total

Imputed social contributions

Total

Social benefits other than social transfers in kind

Other current transfers

Net non-life insurance premiums

Non-life insurance claims

Current transfers within general government

Current international cooperation

$$
\text { from institutions of the EC }
$$

Miscellaneous current transfers

Of which: GNP based fourth own resource

Total, other current transfers

Total resources

Uses

Current taxes on income, wealth etc.

Taxes on income

Other current taxes

Total

Social contributions

Actual social contributions

Employers' actual social contributions

Employees' actual social contributions

Social contributions by self- and non-employed persons

Total actual social contributions

Imputed social contributions

Total

Social benefits other than social transfers in kind

Other current transfers

Net non-life insurance premiums

Non-life insurance claims

Current transfers within general government

Current international cooperation

to institutions of the EC

Miscellaneous current transfers

Of which: GNP based fourth own resource

Total other current transfers

Gross national disposable income

Total uses

After deduction of fixed capital consumption

Disposable income, net

\begin{tabular}{rrrrrr}
149105 & 146612 & 2493 & $\mathbf{1 0 3 2 3 4 3}$ & -8 & \\
185160 & 185160 & & & 565 \\
34888 & 9814 & 25074 & & \\
\hline 220048 & 194974 & 25074 & & 565
\end{tabular}

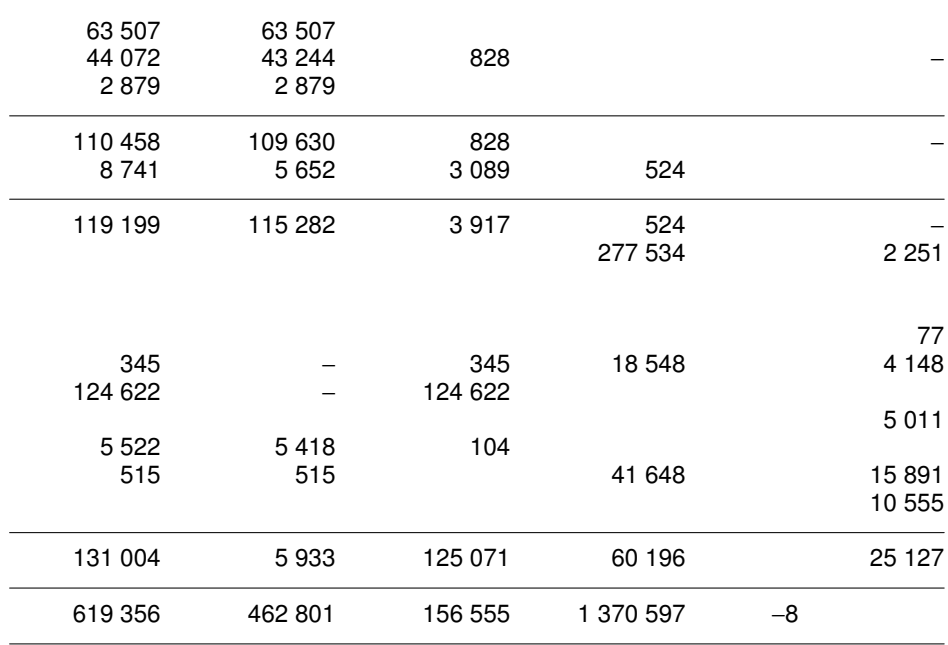

\begin{tabular}{rrrr} 
& & 146111 & 672 \\
1189 & 1189 & 33699 & \\
\hline 1189 & 1189 & 179810 & 672
\end{tabular}

\begin{tabular}{|c|c|c|c|c|c|}
\hline & & & $\begin{array}{r}111115 \\
83428 \\
2879\end{array}$ & & 172 \\
\hline & & & $\begin{array}{r}197422 \\
16594\end{array}$ & & 172 \\
\hline 210806 & 186129 & 24677 & $\begin{array}{r}214016 \\
1020\end{array}$ & & $\begin{array}{l}172 \\
609\end{array}$ \\
\hline 345 & 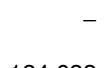 & 345 & 18548 & & $\begin{array}{r}4148 \\
77\end{array}$ \\
\hline 124622 & 124622 & - & & & 5522 \\
\hline $\begin{array}{r}5011 \\
40364 \\
10555\end{array}$ & $\begin{array}{r}5011 \\
40338 \\
10555\end{array}$ & 26 & 14060 & & 3062 \\
\hline 170342 & 169971 & 371 & 32608 & & 12809 \\
\hline 237019 & 106701 & 130318 & 943143 & -8 & \\
\hline 619356 & 462801 & 156555 & 1370597 & -8 & \\
\hline $\begin{array}{l}-14675 \\
222344\end{array}$ & $\begin{array}{l}-7232 \\
99469\end{array}$ & $\begin{array}{r}-7443 \\
122875\end{array}$ & $\begin{array}{l}-47375 \\
895768\end{array}$ & -8 & \\
\hline
\end{tabular}




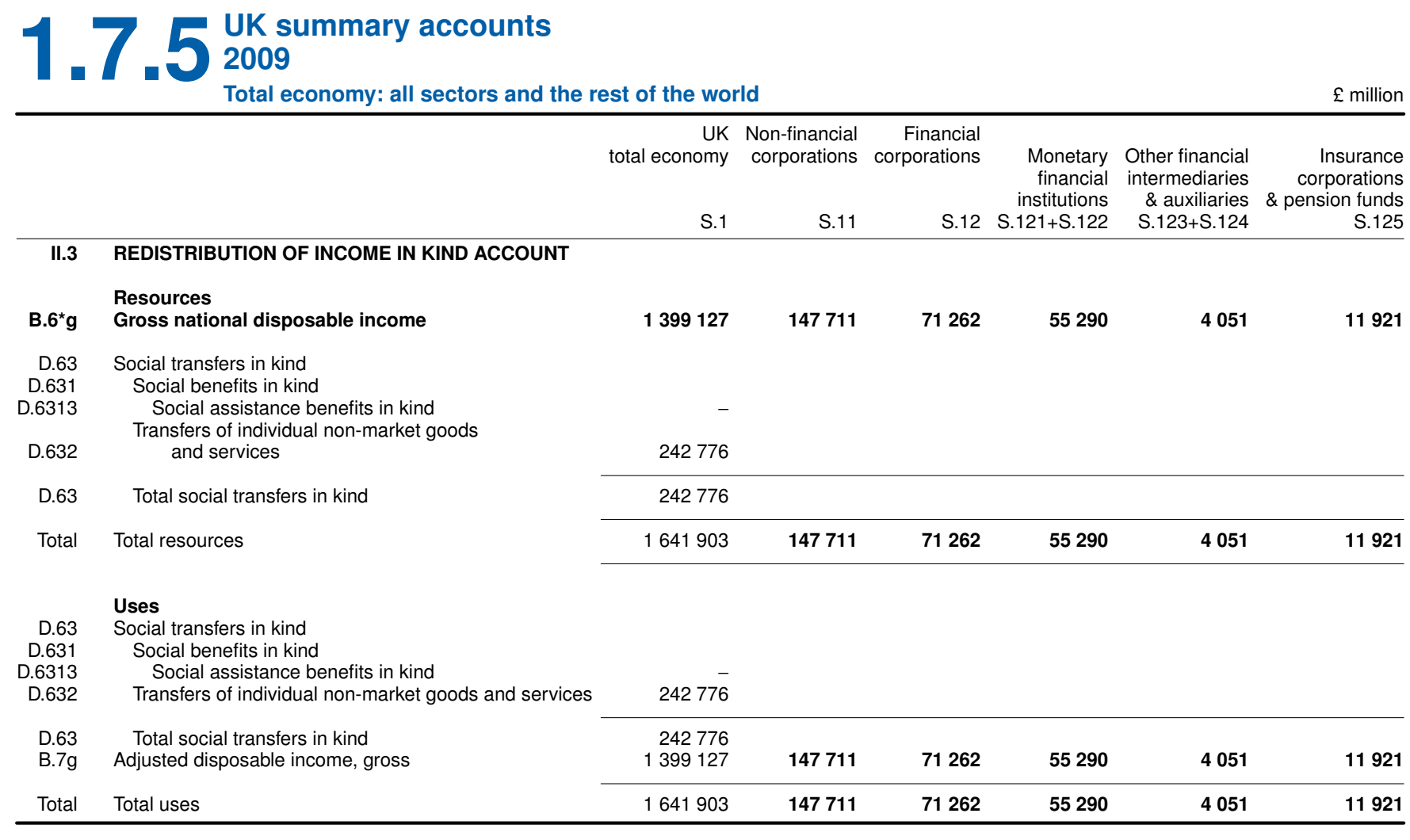




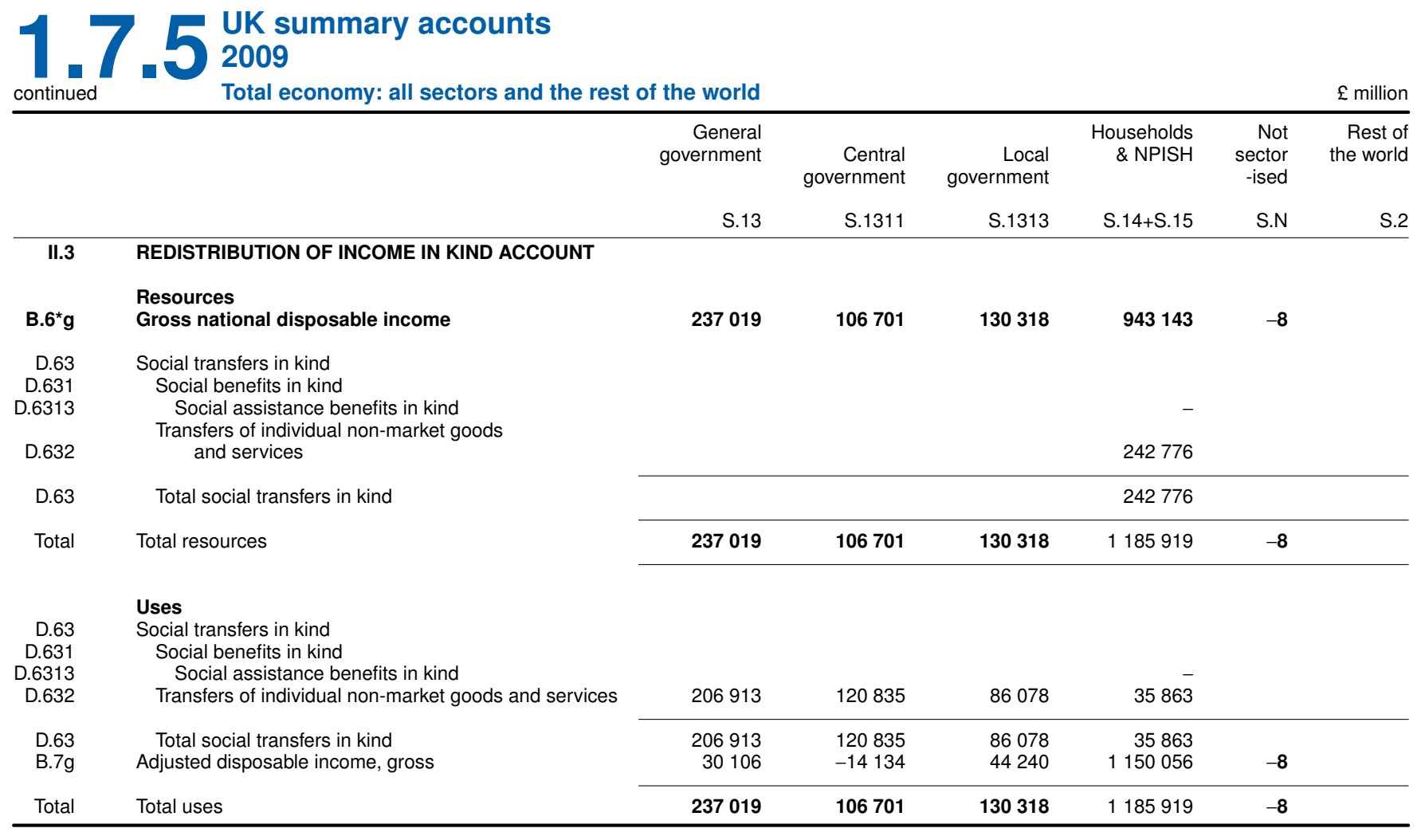




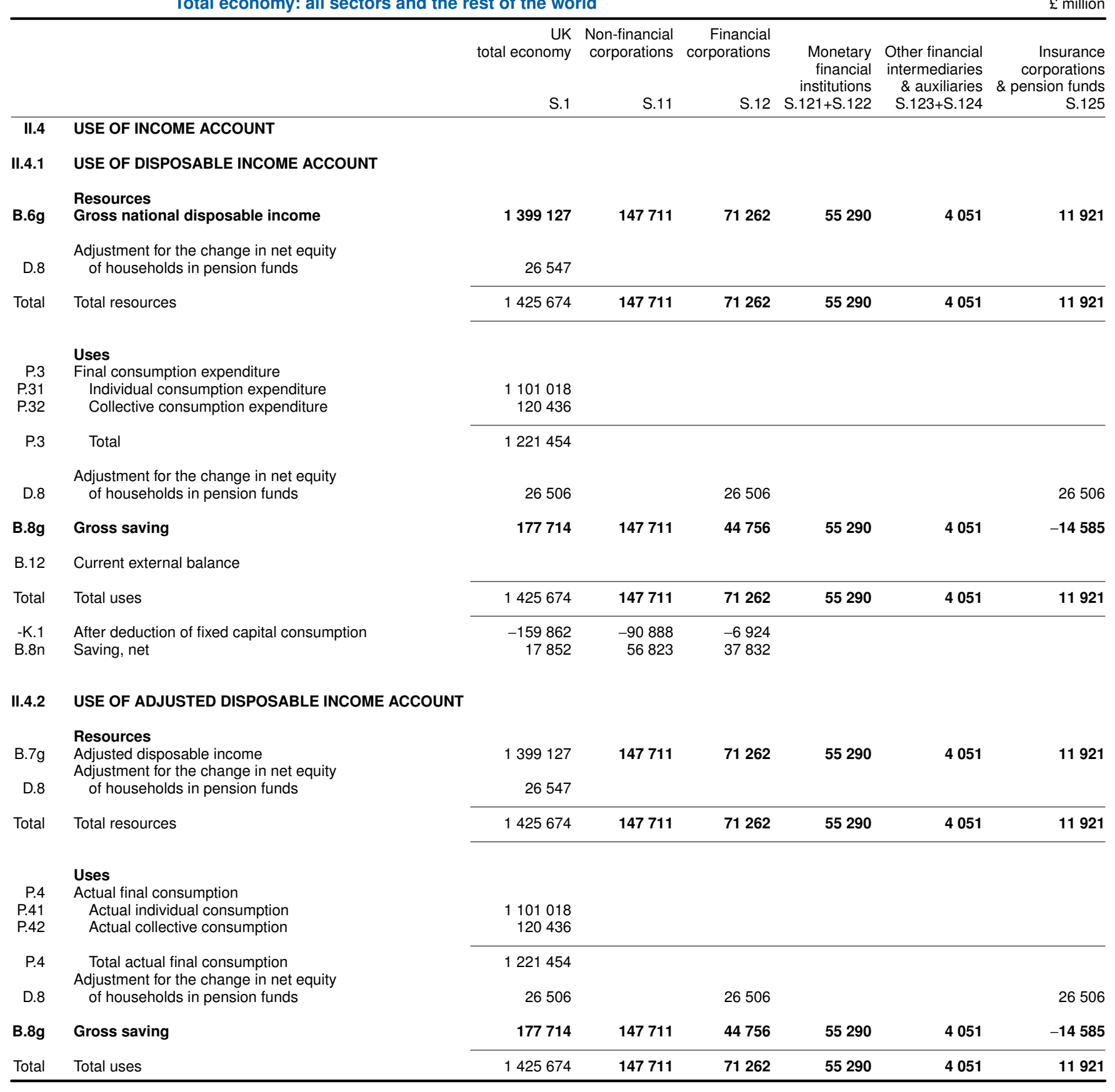


S.13

S.1311

$\mathrm{S} .1313$

S. $14+$ S. 15

S.N S.2

II.4 USE OF INCOME ACCOUNT

\section{II.4.1 USE OF DISPOSABLE INCOME ACCOUNT}

B.6g Gross national disposable income

237019

106701

130318

943143

$-8$

Adjustment for the change in net equity of households in pension funds

Total Total resources

Uses

Final consumption expenditure

Individual consumption expenditure

Collective consumption expenditure

Total

Adjustment for the change in net equity of households in pension funds

Gross saving

$-90330$

$-92948$

2618

75585

\section{B.12 Current external balance}

Total Total uses

-K.1 After deduction of fixed capital consumption

B.8n Saving, net

\begin{tabular}{rrrrr}
\hline $\mathbf{2 3 7 0 1 9}$ & $\mathbf{1 0 6 7 0 1}$ & $\mathbf{1 3 0 3 1 8}$ & 969690 & -8 \\
\hline-14675 & -7232 & -7443 & -47375 & \\
-105005 & -100180 & -4825 & 28210 & -8
\end{tabular}

II.4.2 USE OF ADJUSTED DISPOSABLE INCOME ACCOUNT

Resources

Adjusted disposable income

Adjustment for the change in net equity of households in pension funds

Total resources

$\begin{array}{llllll}30106 & -14134 & 44240 & 1150056 & -8\end{array}$

\begin{tabular}{rrrrr} 
& & 26547 & -41 \\
\hline 30106 & -14134 & 44240 & 1176603 & -8 \\
\hline
\end{tabular}

Uses

Actual final consumption

Actual individual consumption

Actual collective consumption

Total actual final consumption

Adjustment for the change in net equity

of households in pension funds

B.8g Gross saving

Total

Total uses

\begin{tabular}{rrrrr}
120436 & 78814 & 41622 & 1101018 & \\
\hline 120436 & 78814 & 41622 & 1101018 & \\
-90330 & -92948 & 2618 & 75585 & -8 \\
\hline 30106 & -14134 & 44240 & 1176603 & -8 \\
\hline
\end{tabular}




\begin{tabular}{|c|c|c|c|c|c|c|c|}
\hline & & $\begin{array}{r}\text { UK } \\
\text { total economy } \\
\text { S.1 }\end{array}$ & S.11 & S.12 & $\begin{array}{r}\text { Monetary } \\
\text { financial } \\
\text { institutions } \\
\text { S.121+S.122 }\end{array}$ & $\begin{array}{r}\text { Other financial } \\
\text { intermediaries } \\
\& \text { auxiliaries } \\
\text { S.123+S.124 }\end{array}$ & $\begin{array}{r}\text { Insurance } \\
\text { corporations } \\
\& \text { pension funds } \\
\mathrm{S} .125\end{array}$ \\
\hline III & ACCUMULATION ACCOUNTS & & & & & & \\
\hline III.1 & CAPITAL ACCOUNT & & & & & & \\
\hline $\begin{array}{l}\text { B. } 8 \mathrm{~g} \\
\text { B. } 12\end{array}$ & $\begin{array}{l}\text { Changes in liabilities and net worth } \\
\text { Gross Saving } \\
\text { Current external balance }\end{array}$ & 177714 & 147711 & 44756 & 55290 & 4051 & -14585 \\
\hline D.9 & Capital transfers receivable & & & & & & \\
\hline D.91 & Capital taxes & 4206 & & & & & \\
\hline D.92 & Investment grants & 33816 & 12941 & & & & \\
\hline D.9 & Total & 55540 & 13128 & 10120 & 9944 & & 176 \\
\hline $\begin{array}{l}-D .9 \\
-D .91 \\
-D .92 \\
-D .99\end{array}$ & $\begin{array}{l}\text { less Capital transfers payable } \\
\text { Capital taxes } \\
\text { Investment grants } \\
\text { Other capital transfers }\end{array}$ & $\begin{array}{r}-4206 \\
-33225 \\
-14845\end{array}$ & $\begin{array}{r}- \\
-723\end{array}$ & $\begin{array}{r}-1805 \\
-176\end{array}$ & $\begin{array}{r}-1805 \\
-\end{array}$ & & -176 \\
\hline -D.9 & Total & -52276 & -723 & -1981 & -1805 & & -176 \\
\hline B. $10.1 \mathrm{~g}$ & Total change in liabilities and net worth & 180978 & 160116 & 52895 & 63429 & 4051 & -14585 \\
\hline B. $10.1 \mathrm{~g}$ & $\begin{array}{l}\text { Changes in assets } \\
\text { Changes in net worth due to gross saving } \\
\text { and capital transfers }\end{array}$ & 180978 & 160116 & 52895 & 63429 & 4051 & -14585 \\
\hline $\begin{array}{r}-K .1 \\
\text { B. } 10.1 \mathrm{n}\end{array}$ & $\begin{array}{l}\text { After deduction of fixed capital consumption } \\
\text { Changes in net worth due to net saving } \\
\text { and capital transfers }\end{array}$ & $\begin{array}{r}-159862 \\
21116\end{array}$ & $\begin{array}{r}-90888 \\
69228\end{array}$ & $\begin{array}{l}-6924 \\
45971\end{array}$ & & & \\
\hline III.1.2 & $\begin{array}{l}\text { ACQUISITION OF NON-FINANCIAL } \\
\text { ASSETS ACCOUNT }\end{array}$ & & & & & & \\
\hline $\begin{array}{r}\text { B. } 10.1 \mathrm{n} \\
\text { K.1 }\end{array}$ & $\begin{array}{l}\text { Changes in liabilities and net worth } \\
\text { Changes in net worth due to net saving } \\
\text { and capital transfers } \\
\text { Consumption of fixed capital }\end{array}$ & $\begin{array}{r}21116 \\
159862\end{array}$ & $\begin{array}{l}69228 \\
90888\end{array}$ & $\begin{array}{l}45971 \\
6924\end{array}$ & & & \\
\hline B. $10.1 \mathrm{~g}$ & Total change in liabilities and net worth & 180978 & 160116 & 52895 & 63429 & 4051 & -14585 \\
\hline $\begin{array}{r}\text { P.5 } \\
\text { P.51 } \\
\text { P.52 } \\
\text { P.53 }\end{array}$ & $\begin{array}{l}\text { Changes in assets } \\
\text { Gross capital formation } \\
\text { Gross fixed capital formation } \\
\text { Changes in inventories } \\
\text { Acquisitions less disposals of valuables }\end{array}$ & $\begin{array}{r}209253 \\
-11651 \\
429\end{array}$ & $\begin{array}{r}119496 \\
-11391 \\
153\end{array}$ & $\begin{array}{r}6230 \\
53 \\
264\end{array}$ & $\begin{array}{r}4471 \\
27 \\
-\end{array}$ & $\begin{array}{r}1414 \\
- \\
-\end{array}$ & $\begin{array}{r}345 \\
26 \\
264\end{array}$ \\
\hline P.5 & Total & 198035 & 108258 & 6547 & 4498 & 1414 & 635 \\
\hline $\mathrm{K} .2$ & $\begin{array}{l}\text { Acquisitions less disposals of non-produced } \\
\text { non-financial assets }\end{array}$ & -373 & 978 & 16 & - & 28 & -12 \\
\hline de & $\begin{array}{l}\text { Statistical discrepancy between } \\
\text { expenditure components and GDP }\end{array}$ & - & & & & & \\
\hline B.9 & Net lending(+) / net borrowing(-) & -16679 & 50880 & 46332 & 58931 & 2609 & -15208 \\
\hline Total & Total change in assets & 180978 & 160116 & 52895 & 63429 & 4051 & -14585 \\
\hline
\end{tabular}




\begin{tabular}{|c|c|c|c|c|}
\hline $\begin{array}{r}\text { General } \\
\text { government }\end{array}$ & $\begin{array}{r}\text { Central } \\
\text { government }\end{array}$ & $\begin{array}{r}\text { Local } \\
\text { government }\end{array}$ & $\begin{array}{r}\text { Households } \\
\text { \& NPISH }\end{array}$ & $\begin{array}{r}\text { Not } \\
\text { sector } \\
\text {-ised }\end{array}$ \\
\hline
\end{tabular}

\begin{aligned} & \\ \hline III & ACCUMULATION ACCOUNTS \\ III.1 & CAPITAL ACCOUNT \\ III.1.1 & $\begin{array}{c}\text { CHANGE IN NET WORTH DUE TO SAVING } \\ \text { SAVING \& CAPITAL TRANSFERS }\end{array}\end{aligned}$

Changes in liabilities and net worth

$\begin{array}{ll}\text { B.8g } & \text { Gross Saving } \\ \text { B.12 } & \text { Current external balance }\end{array}$

S.13

S. 1311

S.1313

S. $14+$ S. 15 S.2

D.9 Capital transfers receivable

D.91 Capital taxes

D.92 Investment grants

D.99 Other capital transfers

D.9 Total

-D.9 less Capital transfers payable

-D.91 Capital taxes

-D.92 Investment grants

-D.99 Other capital transfers

-D.9 Total

B.10.1g Total change in liabilities and net worth

$\begin{array}{lllll}-90330 & -92948 & 2618 & 75585 & -8\end{array}$

\begin{tabular}{rrrrr}
4206 & 4206 & & & \\
13407 & & 13407 & 7468 & 264 \\
1309 & 322 & 987 & 5902 & 794 \\
\hline 18922 & 4528 & 14394 & 13370 & 1058 \\
\hline
\end{tabular}

\begin{tabular}{|c|c|c|c|c|c|}
\hline $\begin{array}{l}-33225 \\
-11180\end{array}$ & $\begin{array}{l}-30179 \\
-10818\end{array}$ & $\begin{array}{r}-3046 \\
-362\end{array}$ & $\begin{array}{l}-2401 \\
-2766\end{array}$ & & $\begin{array}{r}-855 \\
-3467\end{array}$ \\
\hline-44405 & -40997 & -3408 & -5167 & & -4322 \\
\hline-115813 & -129417 & 13604 & 83788 & -8 & 17052 \\
\hline
\end{tabular}

Changes in assets

B.10.1g Changes in net worth due to gross saving and capital transfers

-K.1 After deduction of fixed capital consumption

B.10.1n Changes in net worth due to net saving and capital transfers

\begin{tabular}{rrrrrr}
-115813 & -129417 & 13604 & 83788 & -8 & 17052 \\
\hline-14675 & -7232 & -7443 & -47375 & & \\
-130488 & -136649 & 6161 & 36413 & -8 &
\end{tabular}

\section{III.1.2 ACQUISITION OF NON-FINANCIAL ASSETS ACCOUNT}

Changes in liabilities and net worth

Changes in net worth due to net saving

and capital transfers

K.1 Consumption of fixed capital

B.10.1g Total change in liabilities and net worth

Changes in assets

Gross capital formation

Gross fixed capital formation

$\begin{array}{ll}\text { P.52 } & \text { Changes in inventories } \\ \text { P.53 } & \text { Acquisitions less disposals of valuables }\end{array}$

$\begin{array}{ll}\text { P.52 Changes in inventories } \\ \text { P.53 } & \text { Acquisitions less disposals of valuables }\end{array}$

P.5 Total

K.2 Acquisitions less disposals of non-produced non-financial assets

\begin{tabular}{rrrrrr}
-130488 & -136649 & 6161 & 36413 & -8 & \\
14675 & 7232 & 7443 & 47375 & & \\
\hline-115813 & -129417 & 13604 & 83788 & -8 & 17052 \\
\hline
\end{tabular}

\begin{tabular}{rrrr}
37125 & 18476 & 18649 & 46401 \\
36 & 36 & - & -349 \\
12 & 12 & & - \\
\hline 37173 & 18524 & 18649 & 46052 \\
-1019 & -63 & -956 & -348
\end{tabular}

\footnotetext{
Statistical discrepancy between
expenditure components and GDP

Statistical discrepancy between
expenditure components and GDP

B.9 Net lending(+) / net borrowing(-)

Total Total change in assets

\begin{tabular}{llllll}
$-\mathbf{1 5 1 9 6 7}$ & $-\mathbf{1 4 7 8 7 8}$ & $\mathbf{- 4 0 8 9}$ & $\mathbf{3 8 0 8 4}$ & $-\mathbf{8}$ & 16679 \\
\hline-115813 & -129417 & 13604 & 83788 & -8 & 17052 \\
\hline
\end{tabular}




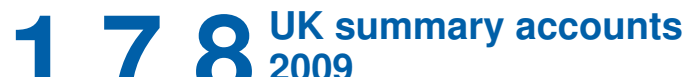

\section{III.2 FINANCIAL ACCOUNT}

\section{F.A Net acquisition of financial assets}

F.1 Monetary gold and special drawing rights (SDRs)

F.2 Currency and deposits

F.21 Currency

F.22 Transferable deposits

F.221 Deposits with UK monetary financial institutions

Deposits with rest of the world monetary financial

institutions

F.29 Other deposits

F.2 Total currency and deposits

F.3 Securities other than shares

F.331 Short term: money market instruments

F.3311 Issued by UK central government

F.3312 Issued by UK local authorities

F.3315 Issued by UK monetary financial institutions

F.3316 Issued by other UK residents

F.3319 Issued by the rest of the world

F.332 Medium (1 to 5 year) and long term (over 5 year) bonds

F.3321

F.3322

F.3325

F.3326

F.3329

F.34

F.3

Issued by UK central government

Issued by UK local authorities

Medium term bonds issued by UK monetary financial institutions

Other medium \& long term bonds issued by UK residents

Long term bonds issued by the rest of the world

Financial derivatives

F.3 Total securities other than shares

F.4 Loans

F.41 Short term loans

F.411 Loans by UK monetary financial institutions,

F.419 Loans by rest of the world monetary financial institutions

F.42 Long term loans

F.421 Direct investment

F.422 Loans secured on dwellings

F.423 Finance leasing

F.424 Other long term loans

F.429 Other long term loans by the rest of the world

F.4 Total loans

F.5 Shares and other equity

F.51 Shares and other equity, excluding mutual funds' shares

F.514 Quoted UK shares

F.515 Unquoted UK shares

F.516 Other UK equity (including direct investment in property)

F.517 UK shares and bonds issued by other UK residents

F.519 Shares and other equity issued by the rest of the world

F.52 Mutual funds' shares

F.521 UK mutual funds' shares

F.529 Rest of the world mutual funds' shares

F.5 Total shares and other equity

F.6 Insurance technical reserves

Net equity of households in life assurance and

F.61 pension funds' reserves

Prepayments of insurance premiums and reserves for

F.62 outstanding claims

F6 Total insurance technical reserves

F.7 Other accounts receivable

F.A Total net acquisition of financial assets
$-132$

\begin{tabular}{rrrrrr}
6548 & 521 & 2476 & 2476 & - & \\
577335 & 20106 & 535802 & 442679 & 95565 & -2442 \\
-217063 & -54252 & -150826 & -147423 & 1570 & -4973 \\
16691 & -309 & 5372 & - & 5372 & - \\
\hline 383511 & -33934 & 392824 & 297732 & 102507 & -7415 \\
\hline
\end{tabular}

\begin{tabular}{rrrrrr}
25388 & -245 & 25556 & 19749 & 3697 & 2110 \\
- & - & - & - & - & \\
-63596 & -62 & -61790 & -40887 & -7695 & -13208 \\
-2729 & -1844 & -924 & -469 & -52 & -403 \\
14204 & 1615 & 12118 & 10690 & 1729 & -301 \\
& & & & & \\
172349 & 677 & 168778 & 209321 & -50227 & 9684 \\
-83 & - & -139 & - & - & -139 \\
33376 & 1438 & 31938 & 10430 & 5424 & 16084 \\
56755 & -3366 & 65711 & 65725 & 7732 & -7746 \\
148024 & -1103 & 141205 & -5939 & 131041 & 16103 \\
-29194 & -3248 & -26938 & -38134 & 10305 & 891 \\
\hline 354494 & -6138 & 355515 & 230486 & 101954 & 23075
\end{tabular}

$-202074$

$-44235$

10527

410

$-40573$

$-275945$

$-43821$

76

$-2568$

-414
9862
486

$-39920$

$-46465$

$-232060$

$-202074$

$-26743$

1196

$\begin{array}{rr}- & 486 \\ 191 & -30155\end{array}$

$-9956$

\section{8}




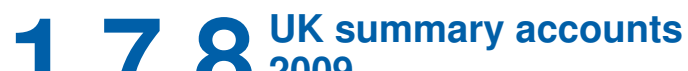

Central
government government

\begin{aligned} \hline III.2 & FINANCIAL ACCOUNT \\ F.A & Net acquisition of financial assets \\ F.1 & Monetary gold and special drawing rights (SDRs) \\ F.2 & Currency and deposits \\ F.21 & Currency \\ F.22 & Transferable deposits \\ F.221 & Deposits with UK monetary financial institutions \\ F.229 & Deposits with rest of the world monetary financial \\ F.29 & institutions \\ F.2 & Tother deposits \\ & \end{aligned}

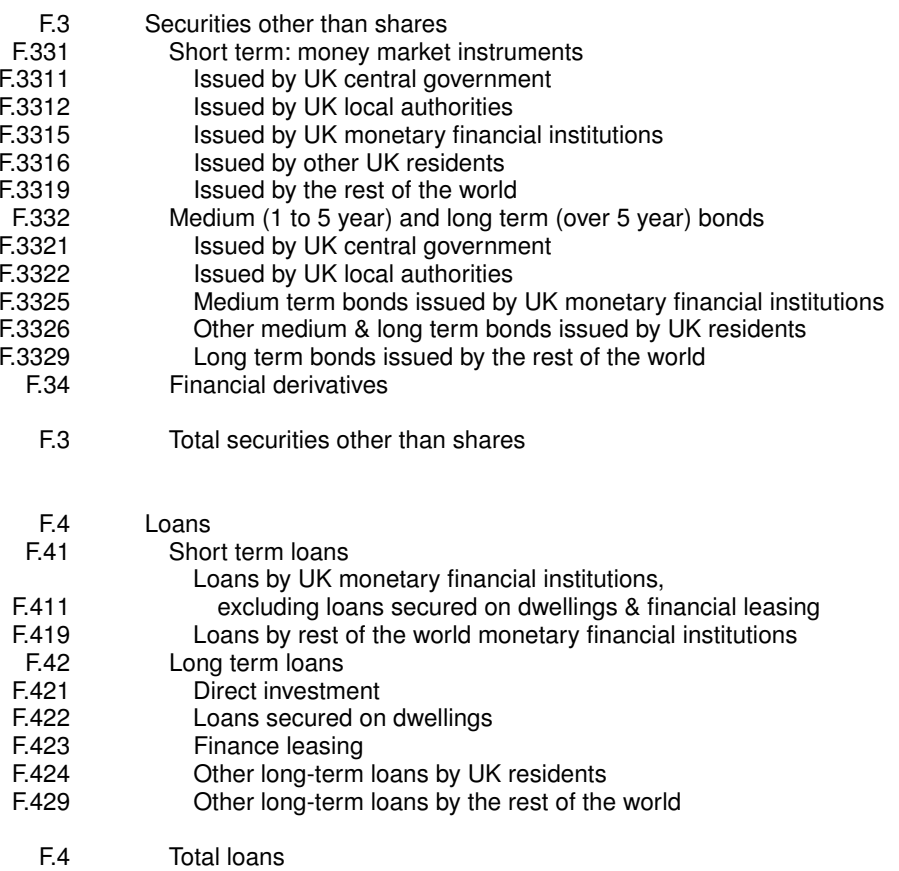

F.5 Shares and other equity

F.51 Shares and other equity, excluding mutual funds' shares

F.514 Quoted UK shares

F.515 Unquoted UK shares

F.516

F.517

F.52

F.52
F.521

F.529

Other UK equity (including direct investment in property)

UK shares and bonds issued by other UK residents

Shares and other equity issued by the rest of the world

Mutual funds' shares

UK mutual funds' shares

Rest of the world mutual funds' shares

\begin{tabular}{rrrrr} 
& & & 3551 & -167 \\
40 & 6958 & -6918 & 21387 & -323972 \\
540 & 540 & & -12525 & \\
9094 & 7075 & 2019 & 2534 & 201 \\
\hline 9674 & 14573 & -4899 & 14947 & -323938 \\
\hline
\end{tabular}

Total shares and other equity

\begin{tabular}{rrrrr}
77 & & 77 & - & 587 \\
- & & & - & \\
-2333 & -2144 & -189 & 589 & 85994 \\
39 & 882 & -843 & - & -5418 \\
471 & 471 & & & \\
28 & & 28 & 2866 & 23529 \\
& & & 56 & - \\
-5236 & -5236 & - & -354 & 7827 \\
7834 & 7834 & & -11 & - \\
1003 & 1003 & & 3867 & 139486 \\
\hline 1883 & 2810 & -927 & 3234 & \\
\hline
\end{tabular}

F.6 Insurance technical reserves

Net equity of households in life assurance and

F.61 pension funds' reserves

Prepayments of insurance premiums and reserves for outstanding claims

F.6 Total insurance technical reserves

665

\begin{tabular}{rrrrr}
3807 & 3688 & 119 & -1892 & 405 \\
\hline 4472 & 3688 & 784 & -1892 & -97701 \\
\hline
\end{tabular}

$-43649$

405

\begin{tabular}{rrrrr}
36800 & 36879 & -79 & 38 & 44790 \\
-16 & -16 & - & -5504 & 48313 \\
-539 & - & -539 & - & 532 \\
- & - & - & - & - \\
1107 & 1107 & & 18298 & 35 \\
& & & -1544 & \\
\hline 37352 & 37970 & -618 & 13568 & 93670 \\
\hline
\end{tabular}




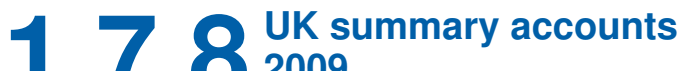

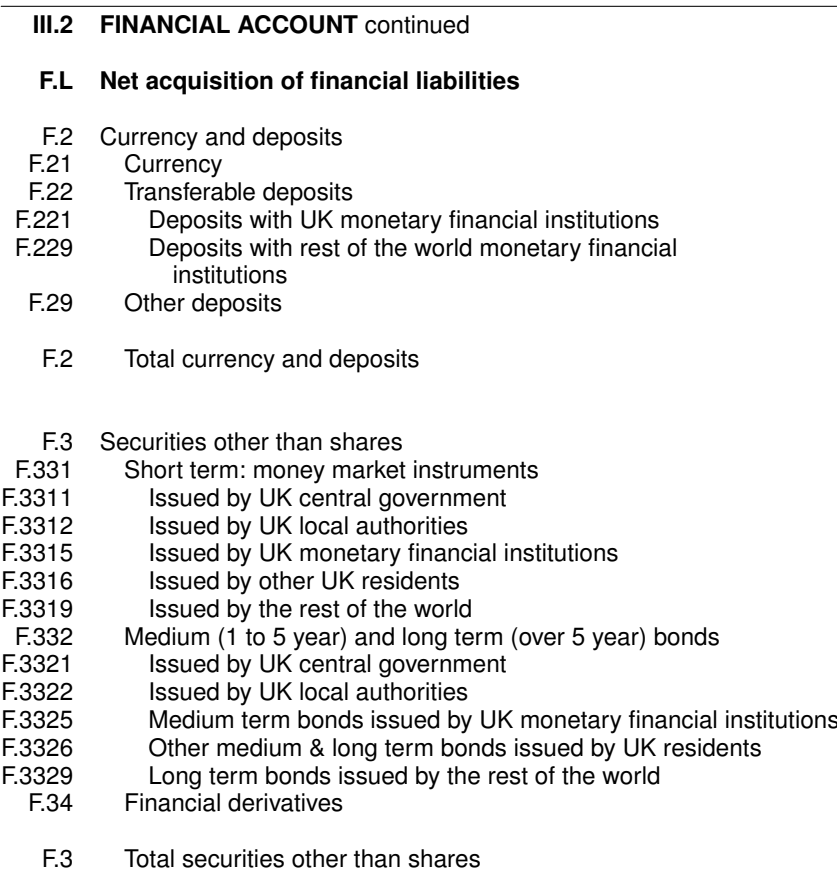

\begin{tabular}{|c|c|c|c|c|}
\hline 6445 & & 6330 & 6330 & \\
\hline 253363 & & 253363 & 253363 & \\
\hline 16892 & - & 7866 & & 7866 \\
\hline 276700 & - & 267559 & 259693 & 7866 \\
\hline
\end{tabular}

F.4 Loans

F.41 Short term loans

Loans by UK monetary financial institutions,

F.411

F.419

excluding loans secured on dwellings \& financial leasing

Loans by rest of the world monetary financial institutions

Long term loans

Direct investment

Loans secured on dwellings

Finance leasing

Other long-term loans by UK residents

Other long-term loans by the rest of the world

F.4 Total loans

S.5 Shares and other equity

F.51 Shares and other equity, excluding mutual funds' shares

F.514 Quoted UK shares

F.515 Unquoted UK shares

F.516 Other UK equity (including direct investment in property)

F.517 UK shares and bonds issued by other UK residents

F.519 Shares and other equity issued by the rest of the world

F.52 Mutual funds' shares

F.521 UK mutual funds' shares

F.529 Rest of the world mutual funds' shares

F.5 Total shares and other equity

25975

$\begin{array}{lllll}22398 & & 22398 & 22398 & \\ -8147 & -5803 & -1781 & & -1781\end{array}$

195878

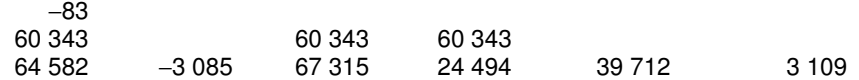

$\begin{array}{lllll}64582 & -3085 & 67315 & 24494 & 39712\end{array}$

\begin{tabular}{llllll}
360946 & -8888 & 148275 & 107235 & 37931 & 3109 \\
\hline
\end{tabular}

F.6 Insurance technical reserves

Net equity of households in life assurance and

F.61 pension funds' reserves

Prepayments of insurance premiums and reserves for

F.62 outstanding claims

F.6 Total insurance technical reserves

\begin{tabular}{rrrrrr}
-84674 & -49705 & -12152 & & -10278 & -1874 \\
-54457 & -42123 & -7499 & & 359 & -7858 \\
& & & & & \\
-43649 & -39053 & -4596 & -144 & -4369 & -83 \\
10527 & 3226 & & & & \\
410 & 219 & 141 & 81 & 60 & \\
-40557 & -27892 & -20600 & & -14297 & -6303 \\
405 & -4 & -116 & & -116 & \\
\hline-211995 & -155332 & -44822 & -63 & -28641 & -16118 \\
\hline
\end{tabular}

F.7 Other accounts payable

F.L Total net acquisition of financial liabilities

$\begin{array}{rrrrrr}120762 & 32864 & 87898 & - & 87383 & 515 \\ 76905 & 13775 & 63130 & -1091 & 63519 & 702 \\ -7 & -7 & - & - & & \\ - & - & - & - & -\end{array}$

$\begin{array}{lll}26271 & 26271 & 26271\end{array}$

\begin{tabular}{llllll}
\hline 223931 & 46632 & 177299 & -1091 & 177173 & 1217 \\
\hline
\end{tabular}

\begin{tabular}{|c|c|c|c|c|c|}
\hline 20408 & & 20408 & & & 20408 \\
\hline-1953 & & -1953 & & & -1953 \\
\hline 18455 & & 18455 & & & 18455 \\
\hline-6788 & -3064 & -2589 & -2591 & -85 & 87 \\
\hline 661249 & -120652 & 564177 & 363183 & 194244 & 6750 \\
\hline
\end{tabular}

\section{B.9 Net lending / borrowing}

F.A Total net acquisition of financial assets

-F.L less Total net acquisition of financial liabilities

B.9f Net lending (+) / net borrowing (-), from financial account

dB.9f Statistical discrepancy between financial \& non-financial accounts

\begin{tabular}{rrrrrr}
636484 & -68394 & 609658 & 411262 & 198536 & -140 \\
-661249 & 120652 & -564177 & -363183 & -194244 & -6750 \\
\hline-24765 & 52258 & 45481 & 48079 & 4292 & -6890 \\
8086 & -1378 & 851 & 10852 & -1683 & -8318 \\
\hline$-16 \mathbf{6 7 9}$ & $\mathbf{5 0 8 8 0}$ & $\mathbf{4 6 3 3 2}$ & $\mathbf{5 8 9 3 1}$ & $\mathbf{2 6 0 9}$ & $\mathbf{- 1 5 2 0 8}$ \\
\hline
\end{tabular}




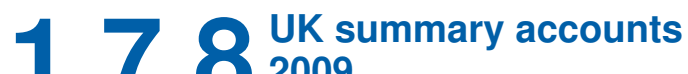

$\begin{array}{rrrrrr}\begin{array}{r}\text { General } \\ \text { government }\end{array} & \begin{array}{r}\text { Central } \\ \text { government }\end{array} & \begin{array}{r}\text { Local } \\ \text { government }\end{array} & \begin{array}{r}\text { Households } \\ \text { \& NPISH }\end{array} & \begin{array}{r}\text { Not } \\ \text { sector } \\ \text {-ised }\end{array} & \begin{array}{r}\text { Rest of } \\ \text { the world }\end{array} \\ \text { S.13 } & \mathrm{S} 1311 & \mathrm{~S} .1313 & \mathrm{~S} .14+\mathrm{S} .15 & \mathrm{SN} & \end{array}$

III.2 FINANCIAL ACCOUNT continued

F.L Net acquisition of financial liabilities

F.2 Currency and deposits

$\begin{aligned} \text { F.21 } & \text { Currency } \\ \text { F.22 } & \text { Transferable deposits }\end{aligned}$

Deposits with rest of the world monetary financial

$$
\text { institutions }
$$

F.29 Other deposits

F.2 Total currency and deposits

\begin{tabular}{lll}
9026 & 9026 & -217063 \\
\hline 9141 & 9141 & -217127 \\
\hline
\end{tabular}

F.3 Securities other than shares

F.331 Short term: money market instruments

F.3311 Issued by UK central government

F3312

F.3315

F.3316

F.3319

F.332

F.3321

F.3322

F.3325

F.3326

F.3329

F.34

Issued by UK local authorities

Issued by UK monetary financial institutions

Issued by other UK residents

Issued by the rest of the world

Medium (1 to 5 year) and long term (over 5 year) bonds

Issued by UK central government

Issued by UK local authorities

Medium term bonds issued by UK monetary financial institutions

Other medium \& long term bonds issued by UK residents

Long term bonds issued by the rest of the world

Financial derivatives

F.41 Short term loans

Loans by UK monetary financial institutions,

excluding loans secured on dwellings \& financial leasing

Loans by rest of the world monetary financial institutions

Long term loans

Direct investment

Loans secured on dwellings

Finance leasing

Other long-term loans by UK residents

Other long-term loans by the rest of the world

F.423

F.424

F.429

Shares and other equity

Shares and other equity, excluding mutual funds' shares

Quoted UK shares

Unquoted UK shares

Other UK equity (including direct investment in property)

UK shares and bonds issued by other UK residents

Shares and other equity issued by the rest of the world

Mutual funds' shares

UK mutual funds' shares

Rest of the world mutual funds' shares

Total shares and other equity

F.6 Insurance technical reserves

Net equity of households in life assurance and

F.61 pension funds' reserves

Prepayments of insurance premiums and reserves for

F.62

outstanding claims

F.6 Total insurance technical reserves

F.7 Other accounts payable

F.L Total net acquisition of financial liabilities

\begin{tabular}{rrrrr}
-20281 & -20507 & 226 & -2536 & -117400 \\
- & - & - & -4835 & \\
& & & & -44235 \\
50 & 50 & - & 7301 & -16 \\
-1788 & -18 & -1770 & 9723 & -161651 \\
525 & 5 & 520 & & -16 \\
\hline-21494 & -20470 & -1024 & 9653 &
\end{tabular}

$221770 \quad 221853 \quad-83 \quad-211$

$\begin{array}{rr}195878 & 195878 \\ -83 & -83\end{array}$ 


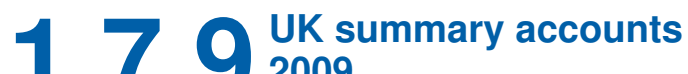

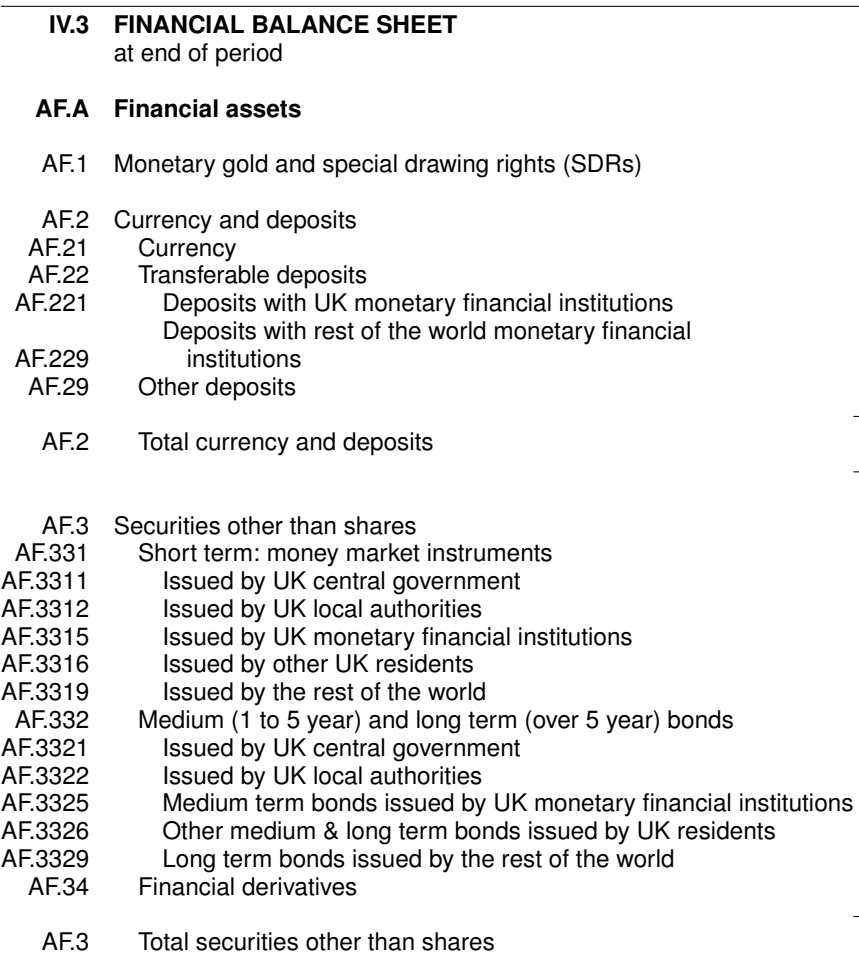

15.7

\begin{tabular}{rrrrrr}
62.1 & 5.2 & 12.8 & 12.7 & 0.1 & \\
3453.5 & 283.4 & 2141.5 & 1142.2 & 909.5 & 89.7 \\
2589.6 & 333.6 & 2186.0 & 1779.3 & 364.2 & 42.5 \\
151.1 & 8.9 & 16.9 & - & 16.9 & - \\
\hline 6256.3 & 631.1 & 4357.2 & 2934.2 & 1290.7 & 132.3 \\
\hline
\end{tabular}

\begin{tabular}{rrrrrr}
38.0 & 0.3 & 37.6 & 22.8 & 11.6 & 3.2 \\
- & - & - & - & - & \\
92.2 & 11.5 & 73.5 & 32.0 & 29.1 & 12.4 \\
12.1 & 6.4 & 4.3 & - & 0.3 & 4.0 \\
85.2 & 2.9 & 76.7 & 65.4 & 7.3 & 4.0 \\
573.4 & 2.6 & 560.6 & 228.8 & 81.4 & 250.4 \\
1.0 & - & 0.5 & - & - & 0.5 \\
181.2 & 4.8 & 176.4 & 54.3 & 31.1 & 91.0 \\
592.7 & 3.6 & 582.9 & 318.8 & 98.6 & 165.6 \\
1176.1 & 18.2 & 1128.6 & 504.0 & 291.5 & 333.1 \\
5275.2 & 25.4 & 5248.5 & 4079.8 & 1072.1 & 96.6 \\
\hline 8027.2 & 75.7 & 7889.6 & 5305.9 & 1622.9 & 960.8 \\
\hline
\end{tabular}

AF.4 Loans

AF.41 Short term loans

Loans by UK monetary financial institutions,

excluding loans secured on dwellings \& financial leasing

Loans by rest of the world monetary financial institutions

AF.419 Long term loans

AF.421 Direct investment

AF.422 Loans secured on dwellings

AF.423 Finance leasing

AF.424 Other long term loans

AF.429 Other long term loans by the rest of the world

AF.4 Total loans

AF.5 Shares and other equity

AF.51 Shares and other equity, excluding mutual funds' shares

AF.514 Quoted UK shares

AF.515 Unquoted UK shares

AF.516 Other UK equity (including direct investment in property)

AF.517 UK shares and bonds issued by other UK residents

AF.519 Shares and other equity issued by the rest of the world

AF.52 Mutual funds' shares

AF.521 UK mutual funds' shares

AF.529 Rest of the world mutual funds' shares

AF.5 Total shares and other equity

2504.8

2504.8

2504.8

\begin{tabular}{rrrrrr}
369.7 & 329.3 & 40.4 & - & 32.4 & 8.0 \\
1234.9 & - & 1230.9 & 922.0 & 306.4 & 2.4 \\
33.7 & 5.0 & 28.7 & 2.6 & 26.1 & \\
307.1 & 65.4 & 130.5 & 4.2 & 24.6 & 101.6 \\
& & & & & \\
\hline 4450.2 & 399.7 & 3935.2 & 3433.7 & 389.5 & 112.0 \\
\hline
\end{tabular}

\begin{tabular}{|c|c|c|c|c|c|}
\hline 845.9 & 48.4 & 589.3 & 22.4 & 215.0 & 351.9 \\
\hline $\begin{array}{l}626.1 \\
119.0\end{array}$ & 72.9 & 326.1 & 113.3 & 207.8 & 4.9 \\
\hline - & - & - & - & - & \\
\hline 1648.7 & 706.3 & 841.8 & 123.4 & - & 386. \\
\hline $\begin{array}{r}504.9 \\
0.8\end{array}$ & 0.4 & 402.6 & 1.3 & 3.5 & 397 \\
\hline 3745.4 & 828.1 & 2159.7 & 260.3 & 758.1 & 1141 \\
\hline
\end{tabular}

AF.6 Insurance technical reserves

Net equity of households in life assurance and

AF.61 pension funds' reserves

Prepayments of insurance premiums and reserves for

AF.62 outstanding claims

AF.6 Total insurance technical reserves

2141.8

$\begin{array}{lll}60.7 & 12.8 & 1.3\end{array}$

0.6

0.7

$\begin{array}{lllll}2202.5 & 12.8 & 1.3 & 0.6 & 0.7\end{array}$

AF.7 Other accounts receivable

399.2

68.2

0.2

17.9

\begin{tabular}{llllll}
25096.5 & 2083.9 & 18411.3 & 11934.3 & 4079.8 & 2397.2 \\
\hline
\end{tabular}




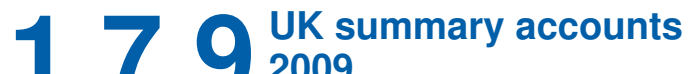

S.13

S.1311

$\mathrm{S} .1313$

S. $14+$ S. 15

\section{IV.3 FINANCIAL BALANCE SHEET at end of period}

\section{AF.A Financial assets}

AF.1 Monetary gold and special drawing rights (SDRs)

AF.2 Currency and deposits

AF.21 Currency

AF.22 Transferable deposits

AF.221 Deposits with UK monetary financial institutions

Deposits with UK monetary financial institutions
Deposits with rest of the world monetary financial

Deposits with
institutions

AF.229

AF.29

AF2

Other deposits

AF.3

AF.331

AF.3311

AF.3312

AF.3315

AF.3316

AF.3319

AF.332

AF.3321

AF 3322

AF.3325

AF.3326

AF.3329

AF.34

AF.3

AF.4

AF.41

AF.411

AF.419

AF.421

AF.422

Total currency and deposits

Securities other than shares

Short term: money market instruments

Issued by UK central government

Issued by UK local authorities

Issued by UK monetary financial institutions

Issued by other UK residents

Issued by the rest of the world

Medium (1 to 5 year) and long term (over 5 year) bonds

Issued by UK central government

Issued by UK local authorities

Medium term bonds issued by UK monetary financial institutions

Other medium \& long term bonds issued by UK residents

Long term bonds issued by the rest of the world

Financial derivatives

Total securities other than shares

\section{Loans}

Short term loans

Loans by UK monetary financial institutions,

excluding loans secured on dwellings \& financial leasing

15.7

\begin{tabular}{rrrrr}
57.2 & 35.2 & 22.0 & 971.5 & 2976.3 \\
4.9 & 4.9 & & 65.1 & \\
26.8 & 21.9 & 4.9 & 98.5 & 1.0 \\
\hline 88.9 & 61.9 & 27.0 & 1179.2 & 2978.7 \\
\hline
\end{tabular}

\begin{tabular}{rrrrr}
0.1 & & 0.1 & - & 19.4 \\
- & & & - & \\
1.6 & 0.4 & 1.2 & 5.6 & 267.8 \\
1.4 & 1.0 & 0.5 & - & 21.5 \\
5.6 & 5.6 & & & \\
0.2 & & 0.2 & 10.0 & 224.3 \\
- & - & & 0.5 & - \\
0.3 & 21.1 & 0.2 & 5.9 & 854.5 \\
21.1 & -1.4 & & 8.2 & \\
-1.4 & 26.8 & 2.2 & 32.9 & 3696.8 \\
\hline 29.0 & & & & 3692.3 \\
\hline
\end{tabular}

\begin{tabular}{rrrrr} 
& & & 2141.8 & 0.2 \\
0.9 & & 0.9 & 45.7 & 10.2 \\
\hline 0.9 & & 0.9 & 2187.5 & 10.4 \\
\hline 71.6 & 71.7 & -0.1 & 123.0 & 2.6 \\
\hline 494.1 & 344.9 & 149.2 & 4107.2 & 9284.5 \\
\hline
\end{tabular}

AF.42

AF.423

AF.424

AF.429

AF.4

Loans by rest of the world monetary financial institutions

Long term loans

Direct investment

Loans secured on dwellings

Finance leasing

Other long-term loans by UK residents

Other long-term loans by the rest of the world

Total loans

Shares and other equity

Shares and other equity, excluding mutual funds' shares

Quoted UK shares

Unquoted UK shares

Other UK equity (including direct investment in property)

UK shares and bonds issued by other UK residents

Shares and other equity issued by the rest of the world

Mutual funds' shares

UK mutual funds' shares

Rest of the world mutual funds' shares

Total shares and other equity

AF.6 Insurance technical reserves

Net equity of households in life assurance and

Net equity of households in life assurance and
pension funds' reserves
Prepayments of insurance premiums and reserves for outstanding claims

AF.62

AF.6 Total insurance technical reserves

795.9

421.9

\begin{tabular}{rrrrr}
4.1 & 0.1 & 4.0 & & \\
92.9 & 92.7 & 0.2 & 18.3 & 4.4 \\
\hline 97.0 & 92.7 & 4.2 & 18.3 & 1222.2 \\
\hline
\end{tabular}

\begin{tabular}{rrrrr}
54.6 & 54.1 & 0.5 & 153.6 & 753.3 \\
6.6 & 5.9 & 0.7 & 220.5 & 599.1 \\
117.7 & 3.8 & 113.9 & 1.4 & 24.5 \\
$-\overline{1}$ & $-\overline{3}$ & - & $-\overline{3}$ & - \\
12.3 & 12.3 & & 88.3 & \\
& & & 101.9 & 1.3 \\
& & & 0.8 & \\
\hline 191.1 & 76.0 & 115.1 & 566.5 & 1378.3 \\
\hline
\end{tabular}

AF.7 Other accounts receivable 


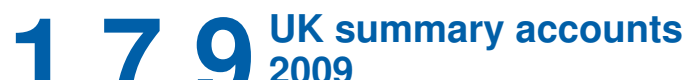

\section{IV.3 FINANCIAL BALANCE SHEET continued at end of period}

\section{AF.L Financial liabilities}

AF.2 Currency and deposits

AF.21 Currency

AF.22 Transferable deposits

AF.221 Deposits with UK monetary financial institutions

Deposits with rest of the world monetary financial

AF.229

AF.29 Other deposits

AF.2 Total currency and deposits

\begin{tabular}{|c|c|c|c|c|}
\hline 62.7 & & 58.7 & 58.7 & \\
\hline 6429.8 & & 6429.8 & 6429.8 & \\
\hline 152.1 & - & 25.2 & & 25.2 \\
\hline 6644.6 & - & 6513.6 & 6488.5 & 25.2 \\
\hline
\end{tabular}

AF.3 Securities other than shares

AF.331 Short term: money market instruments

AF.3311 Issued by UK central government

AF.3312 Issued by UK local authorities

AF.3315 Issued by UK monetary financial institutions

AF.3316 Issued by other UK residents

Issued by the rest of the world

AF.332 Medium (1 to 5 year) and long term (over 5 year) bonds

Medium ( 1 to 5 year) and long term (over 5 year) bonds

Issued by UK central government

Issued by UK local authorities

Medium term bonds issued by UK monetary financial institutions

Other medium \& long term bonds issued by UK residents

Long term bonds issued by the rest of the world
Financial derivatives

AF 3326

AF.34 Financial derivatives

AF.3 Total securities other than shares

AF.4 Loans

AF.41 Short term loans

Loans by UK monetary financial institutions,

excluding loans secured on dwellings \& financial leasing

AF. 411

Loans by rest of the world monetary financial institutions

Long term loans

Direct investment

Loans secured on dwellings

Finance leasing

Other long-term loans by UK residents

Other long-term loans by the rest of the world

AF.423

AF.424

total loans

AF.4 Total loans

\begin{tabular}{rrrrrr}
57.5 & & & & & \\
- & & & & \\
360.1 & & 360.1 & 360.1 & 10.7 & \\
33.6 & 22.5 & 10.7 & & & \\
& & & & & \\
797.7 & & & & & \\
1.0 & & 389.2 & 389.2 & & \\
389.2 & 392.8 & 1050.7 & 271.2 & 778.8 & 93.6 \\
1447.2 & 33.3 & 5158.7 & 4026.8 & 1038.4 & 94.3 \\
5195.6 & 448.6 & 6969.4 & 5047.2 & 1827.9 & \\
\hline 8281.8 & & & & & \\
\hline
\end{tabular}

AF.5 Shares and other equity

AF.51 Shares and other equity, excluding mutual funds' shares

AF514 Quoted UK shares

$\begin{array}{ll}\text { AF.514 } & \text { Quoted UK shares } \\ \text { AF.515 } & \text { Unquoted UK shares }\end{array}$

AF.516 Other UK equity (including direct investment in property)

AF.517 UK shares and bonds issued by other UK residents

AF.519 Shares and other equity issued by the rest of the world

AF.52 Mutual funds' shares

AF.521 UK mutual funds' shares

AF.529 Rest of the world mutual funds' shares

AF.5 Total shares and other equity

\begin{tabular}{rrrrrr}
1601.3 & 479.0 & 925.0 & - & 919.3 & 5.7 \\
795.9 & 183.4 & 586.6 & & 558.5 & 28.1 \\
& & & & & \\
421.9 & 366.9 & 54.9 & 1.0 & 45.2 & 8.7 \\
1234.9 & 43.5 & & & & \\
33.7 & 24.9 & 4.2 & 2.3 & 1.9 & \\
301.2 & 117.7 & 89.4 & - & 88.9 & 0.5 \\
4.4 & 0.9 & 0.5 & & 0.5 & \\
\hline 4393.2 & 1216.2 & 1660.7 & 3.3 & 1614.3 & 43.0 \\
\hline
\end{tabular}

\begin{tabular}{|c|c|c|c|c|c|}
\hline 1599.3 & 1212.6 & 386.6 & 0.1 & 342.6 & 43.9 \\
\hline 1225.2 & 633.5 & 591.7 & 158.7 & 418.6 & 14.4 \\
\hline 143.6 & 143.6 & & & & \\
\hline- & - & - & - & - & - \\
\hline 506.2 & & 506.2 & & 506.2 & \\
\hline 3474.2 & 1989.7 & 1484.5 & 158.8 & 1267.4 & 58.3 \\
\hline
\end{tabular}

AF.6 Insurance technical reserves

Net equity of households in life assurance and

AF.61 pension funds' reserves

Prepayments of insurance premiums and reserves for

AF.62 outstanding claims

$2142.0 \quad 2142.0$

2142.0

AF.6 Total insurance technical reserves

2212.9

2212.9

2212.9

AF.7 Other accounts payable

393.9

161.6

103.6

24.6

2.7

76.3

AF.L Total financial liabilities

$\begin{array}{llllll}25400.6 & 3816.1 & 18944.8 & 11722.4 & 4737.5 & 2484.9\end{array}$

BF.90Net financial assets / liabilities

\begin{tabular}{rrrrrr}
25096.5 & 2083.9 & 18411.3 & 11934.3 & 4079.8 & 2397.2 \\
-25400.6 & -3816.1 & -18944.8 & -11722.4 & -4737.5 & -2484.9 \\
\hline-304.1 & -1732.3 & -533.4 & 212.0 & -657.7 & -87.7 \\
\hline
\end{tabular}




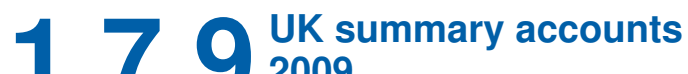

$\begin{array}{rrrrr}\begin{array}{r}\text { General } \\ \text { government }\end{array} & \begin{array}{r}\text { Central } \\ \text { government }\end{array} & \begin{array}{r}\text { Local } \\ \text { government }\end{array} & \begin{array}{r}\text { Households } \\ \text { \&NPISH }\end{array} & \begin{array}{r}\text { Rest of } \\ \text { the world }\end{array} \\ \mathrm{S} .13 & \mathrm{~S} .1311 & \mathrm{~S} .1313 & \mathrm{~S} .14+\mathrm{S} .15 & \mathrm{~S} .2\end{array}$

\begin{tabular}{|c|c|c|c|c|c|c|}
\hline & & S.13 & S.1311 & S.1313 & S.14+S.15 & S.2 \\
\hline IV.3 & $\begin{array}{l}\text { FINANCIAL BALANCE SHEET continued } \\
\text { at end of period }\end{array}$ & & & & & \\
\hline AF.L & Financial liabilities & & & & & \\
\hline AF.2 & Currency and deposits & & & & & \\
\hline AF.21 & Currency & 4.0 & 4.0 & & & 0.8 \\
\hline AF.22 & Transferable deposits & & & & & \\
\hline AF.221 & Deposits with UK monetary financial institutions & & & & & \\
\hline & Deposits with rest of the world monetary financial & & & & & \\
\hline AF.229 & institutions & & & & & 2589.6 \\
\hline AF.29 & Other deposits & 127.0 & 127.0 & & & \\
\hline AF.2 & Total currency and deposits & 131.0 & 131.0 & & & 2590.4 \\
\hline AF.3 & Securities other than shares & & & & & \\
\hline AF.331 & Short term: money market instruments & & & & & \\
\hline AF.3311 & Issued by UK central government & 57.5 & 57.5 & & & \\
\hline AF.3312 & Issued by UK local authorities & - & & - & & \\
\hline AF.3315 & Issued by UK monetary financial institutions & & & & & \\
\hline AF.3316 & Issued by other UK residents & & & & 0.4 & \\
\hline AF.3319 & Issued by the rest of the world & & & & & 85.2 \\
\hline AF.332 & Medium ( 1 to 5 year) and long term (over 5 year) bonds & & & & & \\
\hline AF.3321 & Issued by UK central government & 797.7 & 797.7 & & & \\
\hline AF.3322 & Issued by UK local authorities & 1.0 & & 1.0 & & \\
\hline AF.3325 & Medium term bonds issued by UK monetary financial institutions & & & & & \\
\hline AF.3326 & Other medium \& long term bonds issued by UK residents & & & - & 3.7 & \\
\hline AF.3329 & Long term bonds issued by the rest of the world & & & & & 1176.1 \\
\hline AF.34 & Financial derivatives & & & & 3.5 & 2176.4 \\
\hline AF.3 & Total securities other than shares & 856.1 & 855.1 & 1.0 & 7.6 & 3437.7 \\
\hline AF.4 & Loans & & & & & \\
\hline AF.41 & Short term loans & & & & & \\
\hline & Loans by UK monetary financial institutions, & & & & & \\
\hline AF.411 & excluding loans secured on dwellings \& financial leasing & 16.1 & 5.0 & 11.0 & 181.2 & 903.5 \\
\hline AF.419 & Loans by rest of the world monetary financial institutions & - & - & - & 25.9 & \\
\hline AF.42 & Long term loans & & & & & \\
\hline AF.421 & Direct investment & & & & & 369.7 \\
\hline AF 422 & Loans secured on dwellings & & & & 11914 & \\
\hline AF.423 & Finance leasing & 4.6 & 4.5 & 0.1 & & - \\
\hline AF.424 & Other long-term loans by UK residents & 52.0 & - & 52.0 & 42.1 & 5.9 \\
\hline AF.429 & Other long-term loans by the rest of the world & 3.0 & - & 3.0 & & \\
\hline AF.4 & Total loans & 75.8 & 9.6 & 66.2 & 1440.6 & 1279.1 \\
\hline
\end{tabular}

Shares and other equity

Shares and other equity, excluding mutual funds' shares

Quoted UK shares

Unquoted UK shares

Other UK equity (including direct investment in property)

UK shares and bonds issued by other UK residents

Shares and other equity issued by the rest of the world

Mutual funds' shares

UK mutual funds' shares

Rest of the world mutual funds' shares

Total shares and other equity

Insurance technical reserves

Net equity of households in life assurance and

pension funds' reserves

AF.6

Prepayments of insurance premiums and reserves for outstanding claims

Total insurance technical reserves

AF.7 Other accounts payable

\section{Total financial liabilities}

\begin{tabular}{rrrrr}
45.3 & 36.7 & 8.6 & 83.4 & 8.0 \\
\hline 1108.1 & 1032.4 & 75.8 & 1531.6 & 8964.7 \\
\hline
\end{tabular}

\section{Net financial assets / liabilities}

AF.A Total financial assets

-AF.L less Total financial liabilities

\begin{tabular}{rrrrr}
494.1 & 344.9 & 149.2 & 4107.2 & 9284.5 \\
-1108.1 & -1032.4 & -75.8 & -1531.6 & -8964.7 \\
\hline-614.0 & -687.4 & 73.4 & 2575.7 & 319.8 \\
\hline
\end{tabular}




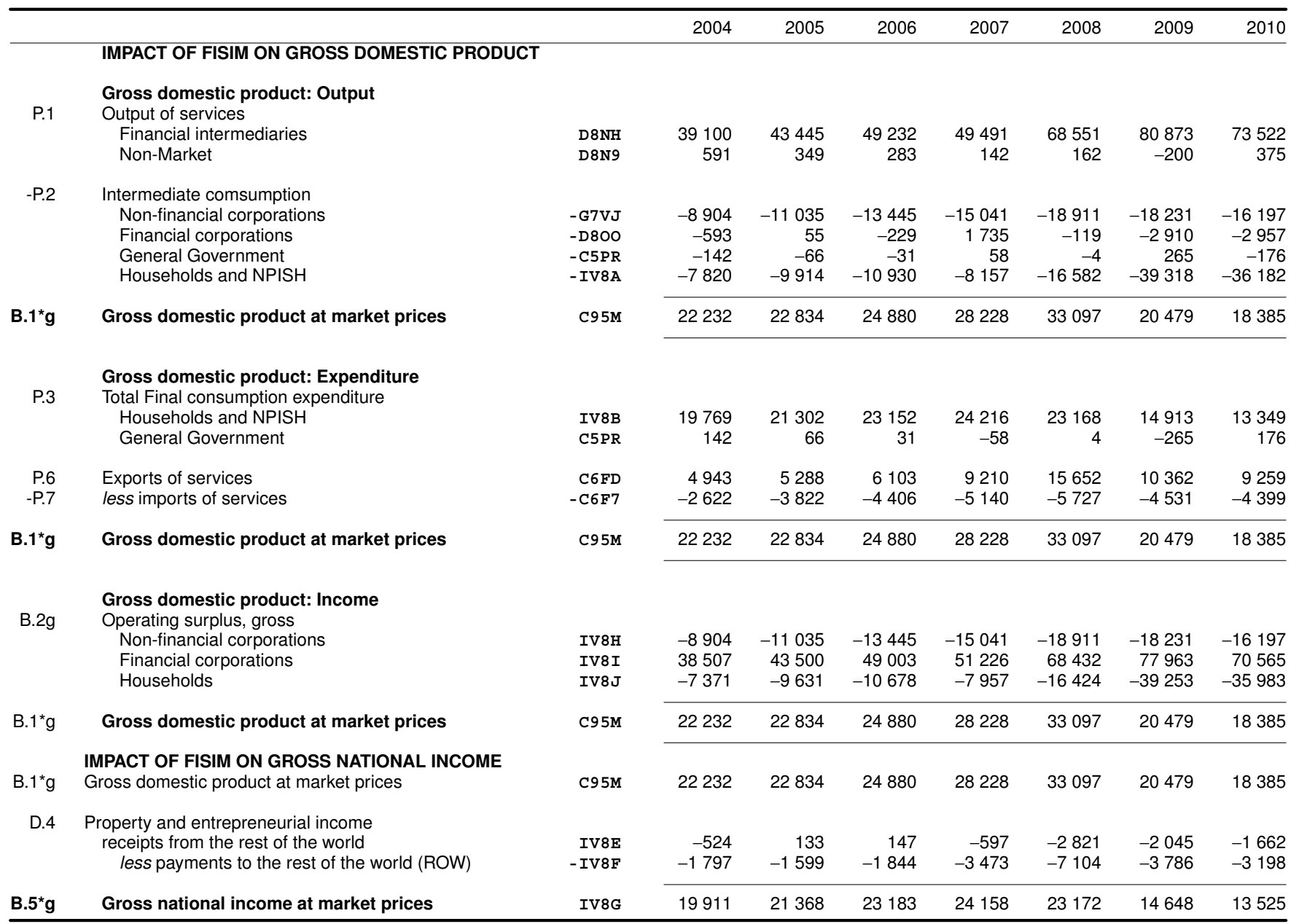

1 FISIM = Financial intermediation services indirectly measured

2 In some sectors, including central government and local government, FISIM has been adjusted to avoid negative values for 2008. As 2008 is the reference year, negative values could distort the chained volume measures. The overall impact of these adjustments is small. A joint UN/Eurostat task force is reviewing the methodology for measurement of FISIM.

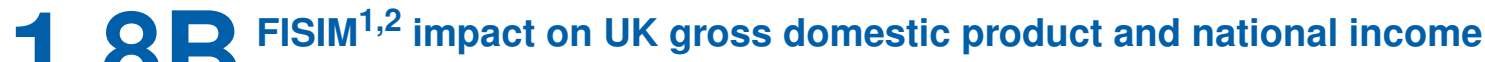 Chained volume measures (reference year 2008)}

$£$ million

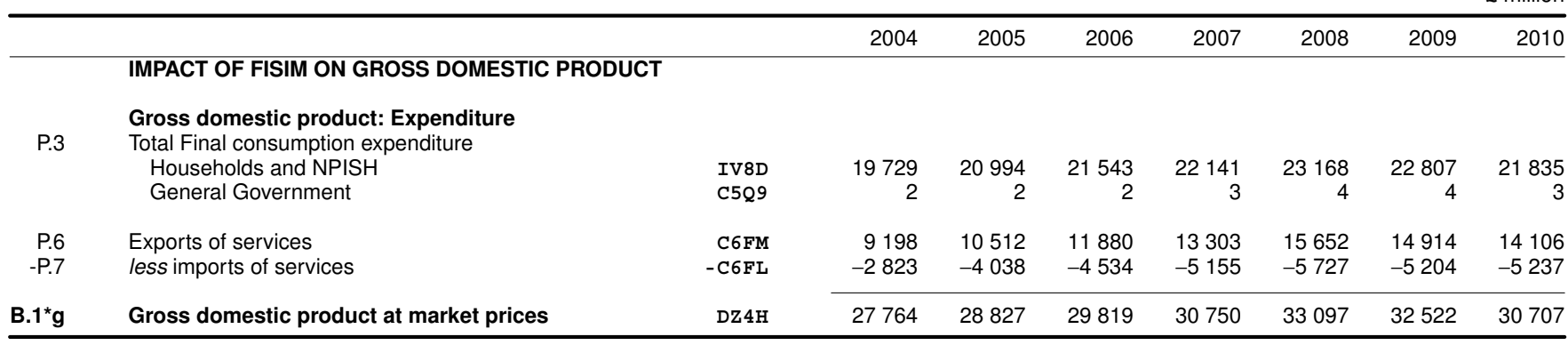

$1 \mathrm{FISIM}=$ Financial intermediation services indirectly measured

2 In some sectors, including central government and local government, FISIM

has been adjusted to avoid negative values for 2008. As 2008 is the refer-

ence year, negative values could distort the chained volume measures. The

overall impact of these adjustments is small. A joint UN/Eurostat task force

is reviewing the methodology for measurement of FISIM. 


\begin{tabular}{|c|c|c|c|c|c|c|c|c|c|}
\hline & & & 2004 & 2005 & 2006 & 2007 & 2008 & 2009 & 2010 \\
\hline & \multicolumn{9}{|l|}{ Public corporations } \\
\hline & Unadjusted interest received & NENH & 1263 & 1788 & 874 & 729 & 844 & 342 & 321 \\
\hline & plus FISIM & C7RL & -2 & 4 & 5 & 2 & 2 & -29 & -17 \\
\hline \multirow[t]{4}{*}{ D.41 } & Interest received & CPBV & 1261 & 1792 & 879 & 731 & $84 \overline{6}$ & 313 & 304 \\
\hline & Uses & & & & & & & & \\
\hline & Unadjusted interest paid & NENG & 1363 & 1272 & 1435 & 1587 & 1792 & 1616 & 1332 \\
\hline & less FISIM & $\mathrm{D} 8 \mathrm{KD}$ & 27 & 21 & 41 & 74 & 47 & 67 & 42 \\
\hline & $\begin{array}{l}\text { Private non-financial corporations } \\
\text { Resources }\end{array}$ & & & & & & & & \\
\hline & Unadjusted interest received & I69R & 9917 & 12075 & 19993 & 24868 & 19348 & 5640 & 4875 \\
\hline & plus FISIM & IV87 & 2963 & 3513 & 4519 & 4922 & 4372 & 1446 & 1741 \\
\hline \multirow[t]{4}{*}{ D.41 } & Interest received & DSZR & 12880 & 15588 & 24512 & 29790 & 23720 & 7086 & 6616 \\
\hline & Uses & & & & & & & & \\
\hline & Unadjusted interest paid & I6A2 & 39392 & 45324 & 51058 & 63548 & 65551 & 47075 & 37569 \\
\hline & less FISIM & IV86 & 5916 & 7497 & 8880 & 10043 & 14490 & 16747 & 14431 \\
\hline \multirow{3}{*}{ D.41 } & $\begin{array}{l}\text { Non-financial corporations } \\
\text { Resources }\end{array}$ & & & & & & & & \\
\hline & Unadjusted interest received & J4WQ & 11180 & 13863 & 20867 & 25597 & 20192 & 5982 & 5196 \\
\hline & plus FISIM & IV89 & 2961 & 3517 & 4524 & 4924 & 4374 & 1417 & 1724 \\
\hline \multirow[t]{4}{*}{ D.41 } & Interest received & EABC & 14141 & 17380 & 25391 & 30521 & 24566 & 7399 & 6920 \\
\hline & Uses & & & & & & & & \\
\hline & Unadjusted interest paid & J4WS & 40755 & 46596 & 52493 & 65135 & 67343 & 48691 & 38901 \\
\hline & less FISIM & IV88 & 5943 & 7518 & 8921 & 10117 & 14537 & 16814 & 14473 \\
\hline \multirow[t]{5}{*}{ D.41 } & Interest paid & EABG & 34812 & 39078 & 43572 & 55018 & 52806 & 31877 & 24428 \\
\hline & Financial corporations & & & & & & & & \\
\hline & Resources & & & & & & & & \\
\hline & Unadjusted interest received & J4WU & 221186 & 277738 & 367636 & 468157 & 483971 & 268781 & 229259 \\
\hline & plus FISIM & IV8Y & -25834 & -31031 & -34431 & -32377 & -50988 & -84101 & -75881 \\
\hline \multirow[t]{4}{*}{ D.41 } & Interest received & NHCK & 195352 & 246707 & 333205 & 435780 & 432983 & 184680 & 153378 \\
\hline & Uses & & & & & & & & \\
\hline & Unadjusted interest paid & J4WW & 147137 & 200346 & 287265 & 376128 & 375751 & 167774 & 127919 \\
\hline & plus FISIM & IV8z & 12673 & 12469 & 14572 & 18849 & 17444 & -6138 & -5316 \\
\hline \multirow[t]{3}{*}{ D.41 } & Interest paid & NHCM & 159810 & 212815 & 301837 & 394977 & 393195 & 161636 & 122603 \\
\hline & Unadjusted interest received & I69N & 5716 & 5364 & 5917 & 6628 & 7503 & 7252 & 5402 \\
\hline & plus FISIM & $\mathrm{C} 6 \mathrm{GA}$ & 39 & 37 & 16 & -58 & 1 & -258 & -97 \\
\hline \multirow[t]{4}{*}{ D.41 } & Interest received & NMCE & 5755 & 5401 & 5933 & 6570 & 7504 & 6994 & 5305 \\
\hline & Uses & & & & & & & & \\
\hline & Unadjusted interest paid & I69W & 23224 & 25935 & 27463 & 31313 & 32788 & 26982 & 42235 \\
\hline & less FISIM & C6G9 & 12 & 28 & 18 & 44 & 1 & 34 & 68 \\
\hline D.41 & Interest paid & NUHA & 23212 & 25907 & 27445 & 31269 & 32787 & 26948 & 42167 \\
\hline & $\begin{array}{l}\text { Local government } \\
\text { Resources }\end{array}$ & & & & & & & & \\
\hline & Unadjusted interest received & I690 & 1013 & 1075 & 1544 & 1965 & 2661 & 1019 & 687 \\
\hline & plus FISIM & $\mathrm{C} 6 \mathrm{FQ}$ & 70 & -5 & -12 & 9 & 1 & -387 & -146 \\
\hline D.41 & Interest received & NMKB & 1083 & 1070 & 1532 & 1974 & 2662 & 632 & 541 \\
\hline & Uses & & & & & & & & \\
\hline & Unadjusted interest paid & I69X & 3782 & 3475 & 3540 & 3470 & 3715 & 3571 & 3500 \\
\hline & less FISIM & C6FP & 21 & 6 & 9 & -53 & 1 & 346 & 351 \\
\hline D.41 & Interest paid & NCBW & 3761 & 3469 & 3531 & 3523 & 3714 & 3225 & 3149 \\
\hline & $\begin{array}{l}\text { Households \& non-profit institutions serving ho } \\
\text { Resources }\end{array}$ & & & & & & & & \\
\hline & Unadjusted interest received & J4WY & 26636 & 32350 & 34677 & 43421 & 42718 & 20143 & 19212 \\
\hline & plus FISIM & IV8W & 8169 & 7982 & 9087 & 11656 & 8078 & -10371 & -9887 \\
\hline D.41 & Interest received & QWLZ & 34805 & 40332 & 43764 & 55077 & 50796 & 9772 & 9325 \\
\hline & Uses & & & & & & & & \\
\hline & Unadjusted interest paid & J4WZ & 62709 & 73809 & 80725 & 95118 & 98921 & 72016 & 64753 \\
\hline & less FISIM & IV8x & 18971 & 22951 & 24743 & 20517 & 31514 & 64537 & 59219 \\
\hline D.41 & Interest paid & QWMG & 43738 & 50858 & 55982 & 74601 & 67407 & 7479 & 5534 \\
\hline & Rest of the world & & & & & & & & \\
\hline & Resources & & & & & & & & \\
\hline & Unadjusted interest received & $169 \mathrm{~V}$ & 76886 & 112129 & 156724 & 203820 & 196884 & 102439 & 86407 \\
\hline & FISIM on interest paid to Rest of World & IV8F & 1797 & 1599 & 1844 & 3473 & 7104 & 3786 & 3198 \\
\hline D.41 & Interest received & QYNG & 78683 & 113728 & 158568 & 207293 & 203988 & 106225 & 89605 \\
\hline & Uses & & & & & & & & \\
\hline & Unadjusted interest paid & I6A6 & 65010 & 92358 & 135879 & 178424 & 175411 & 86582 & 68855 \\
\hline & FISIM on interest received from Rest of World & IV8E & -524 & 133 & 147 & -597 & -2821 & -2045 & -1662 \\
\hline D.41 & Interest paid & QYNJ & 64486 & 92491 & 136026 & 177827 & 172590 & 84537 & 67193 \\
\hline
\end{tabular}

1 FISIM = Financial intermediation services indirectly measured

2 In some sectors, including central government and local government, FISIM has been adjusted to avoid negative values for 2008. As 2008 is the reference year, negative values could distort the chained volume measures. The overall impact of these adjustments is small. A joint UN/Eurostat task force is reviewing the methodology for measurement of FISIM.

3 Interest is recorded within the allocation of primary income account 